$8 \times 3 x^{2}$

\title{
800
$=$
48
}

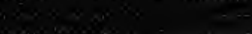

$\cos 20 x$
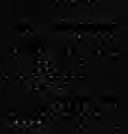

30.0

\section{$\lim _{20}$}




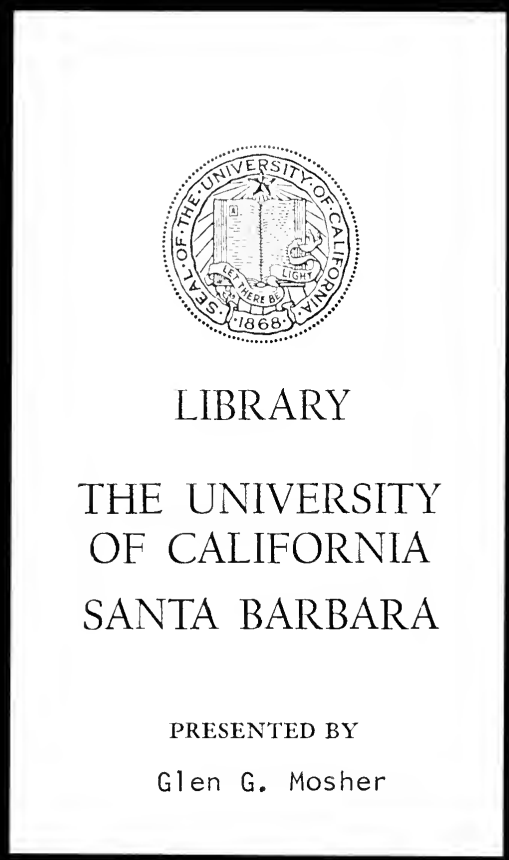




$580-$

Priser Sovee M.9.T-o?.

$7 e 617$. 1571 Beacou Jt. 



\section{GRAPHIC STATICS}

WITH APPLICATIONS TO

TRUSSES, BEAMS, AND ARCHES.

BY

JEROME SONDERICKER, B.S., C.E.,

Associate Professor of Aphlied Mechanics,

Massachusetts Institute of Technology.

FIRSTEDITION.

FIRST THOUSAND.

NEW YORK :

JOHN WILEY \& SONS.

LONDON: CHAPMAN \& HALL, LIMITED.

I904. 
Copyright, 1903,

BY

JEROME SONDERICKER.

ROBERT DRUMMOND, PRINTER, NEW YORK. 


\section{PREFACE.}

THIs book is the outgrowth of an extended experience in teaching Graphic Statics at the Massachusetts Institute of Tech. nology. While it deals specifically with problems encountered in building construction, it should be found serviceable to engineers and engineering students generally.

As preparation, the reader should have a knowledge of Statics and Strength of Materials, including beam stresses and deflections, as these subjects are commonly presented.

To be successful in the employment of graphical methods, it is necessary not only to understand the general principles involved, but also to know how to proceed in the construction of the drawings, in order to secure the most satisfactory results. This matter is given more attention than usual.

An attempt has been made in $\S 6$, Chapter II, to develop a general method of dealing with frames where bending stresses occur in addition to the tension and compression stresses. It is hoped this may be found useful.

\section{JEROME SONDERICKER.}

MASSACHUSETTS INSTItUTE OF TEChNOLOGY,

May, 1903.

iii 



\section{CONTENTS.}

\section{CHAPTER I.}

\section{GENERAL METHODS}

§. INTRODUCTION.

ART.

I. Definition of Graphic Statics.......................... I

2. Representation of Forces.......................... I

3. Resultant of Forces lying in the Same Plane.................. I

4. Example...................................... 3

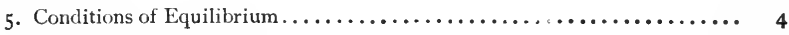

6. Examples and Problems............................. 5

§2. FUNICULAR POLYGoN.

7. Definitions.................................... 8

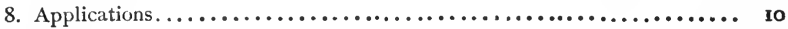

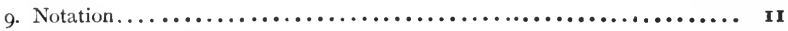

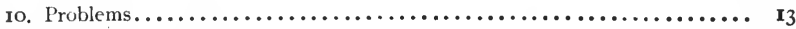

I I. Distributed Forces.............................. 14

12. Funicular Polygon for a Distributed Force................. 14

13. Funicular Polygon for a Uniformly Distributed Force............ 15

§ 3. FORCES IN EQUILIBRIUM.

14. Use of Funicular Polygon......................... I7

15. Case I. Parallel Forces......................... 17

16. Case II. Non-parallel Forces.......................... 19

17. Case III. Non-parallel Forces....................... 20

18. Resolution of Forces into Components................... 2 I

§ 4. GRAPHICAL DETERMINATION OF MOMENTS.

19. Moment of Resultant of any System of Forces............... 2 I

20. Moment of Resultant of Parallel Forces.................... 22 
§5. SOME SPECIAL CONSTRUCTIONS FOR FUNICULAR POLYGONS.

ART.

21. Locus of Points of Intersection of Corresponding Strings........... 23

22. Locus of Poles of Funicular Polygons passing through Two Given Points, 24

23. Funicular Polygon through Three Points.................. 25

24. Funicular Polygon through Three Points. Parallel Forces.......... 25

25. Funicular Polygon through Two Points, One String having a Given Direction.

\section{CHAP'TER II.}

ROOF-TRUSSES.

§ I. CONSTRUCTION OF ROOFS; LOADS, ETC.

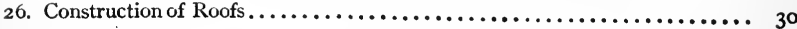

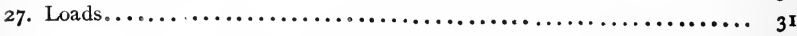

§ 2. DETERMINATION OF REACTIONS OF SUPPORTS.

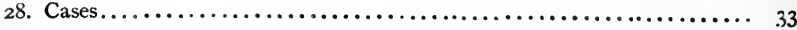

29. Examples...................................... 35

3o. Algebraic Solution. ............................... 37

§ 3. DETERMINATION OF STRESSES.

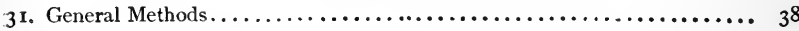

32. Method of Sections................................... $3^{8}$

33. Method of Joints................................ 39

34. Determination of Stresses in Roof-trusses................... 40

35. Example. Bow's Notation........................... 40

36. Determination of Maximum and Minimum Stresses.............. 44

37. Example...................................... 45

38. General Remarks................................. 49

39. Problems...................................... $5^{\text {I }}$

40. Cantilever Truss.................................. 53

4I. Trusses having only Two Forces to determine at Each Joint........ 53

42. Fink, or French, Roof-truss......................... 53

§ 4. COUNTERBRACING AND DOUBLE SYSTEMS OF BRACING.

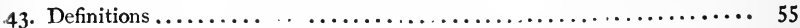

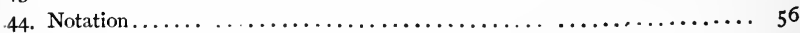

45. Determination of Stresses. Diagrams drawn for Combined Loads...... $5^{6}$

46. Example.................................... 60

47. Determination of Stresses in Trusses with Counterbracing. Second Method 62 
48. Trusses having a Double System of Web Members.............. 64

49. Double Diagonal Bracing................................... $6_{5}$

\section{§ 5. THREE-HINGED ARCH.}

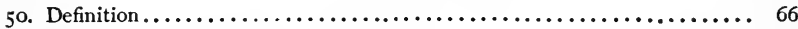

5 r. Determination of Reactions of Hinges.................... 66

52. Determination of Reactions. Algebraic Solution............... 68

53. Determination of Stresses in Braced Arches................. 69

54. Three-hinged Arch. Solid Ribs. Determination of Stresses........ 72

55. Bending Moments proportional to Vertical Intercepts........... 74

\section{§ 6. BENDING S'TRESSES, SWAY-BRACING.}

56. Conditions under which Bending Stresses occur............. 75

57. Purlins supported at Other Points than the Joints.............. 76

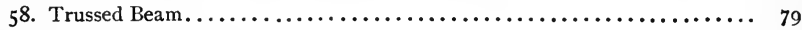

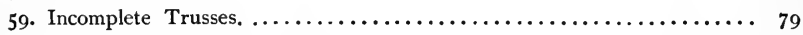

60. Trusses supported by Columns....................... 82

6r. Case I. Columns hinged at Base....................... 82

62. Case II. Columns hinged at Base and loaded only at the Joints....... 87

63. Case III. Columns fixed at Base........................... 87

64. Case IV. Columns fixed at Base and loaded only at the Joints........ 89

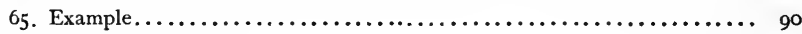

66. Approximate Solution. . . . . . . . . . . .

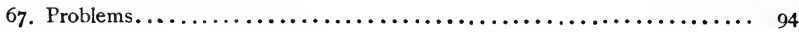

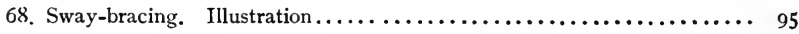

6. Example.................................. 96

\section{CHAPTER III.}

BEAMS.

§ I. SHEARING FORCE AND BENDING MOMENT.

7o. Definitions...................................... $9^{8}$

71. Graphical Representation of Shearing Force and Bending Moment..... 98

72. Relation between Shear and Moment Diagrams............... roo

73. Relation between Moment Diagram and Elastic Curve............. ror

74. Examples.................................... r

75. Problems $\ldots \ldots \ldots \ldots \ldots \ldots \ldots \ldots \ldots \ldots \ldots \ldots \ldots \ldots \ldots \ldots \ldots \ldots \ldots \ldots \ldots \ldots \ldots \ldots$ ro6 
ART.

§ 2. DEFLECTION OF BEAMS.

76. Graphical Determination of Elastic Curve................. I07

77. Examples...................................... 1

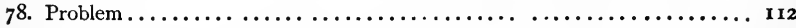

79. Center of Gravity of Any Quadrilateral Area $\ldots \ldots \ldots \ldots \ldots \ldots \ldots \ldots$ I 12

\section{CHAPTER IV.}

MASONRY ARCHES, ABUTMENTS, ETC.

§ I. GENERAL CONDITIONS OF STABILITY.

80. Nature of the Forces involved $\ldots \ldots \ldots \ldots \ldots \ldots \ldots \ldots \ldots \ldots \ldots$ II 4

81. Resistance of a Masonry Joint...................... 115

82. Resistance to Overturning $\ldots \ldots \ldots \ldots \ldots \ldots \ldots \ldots \ldots \ldots \ldots \ldots \ldots \ldots \ldots \ldots \ldots \ldots \ldots \ldots$

83. Resistance to Sliding............................. 115

84. Resistance to Crushing $\ldots \ldots \ldots \ldots \ldots \ldots \ldots \ldots \ldots \ldots \ldots \ldots \ldots \ldots \ldots \ldots \ldots \ldots \ldots \ldots \ldots \ldots \ldots$

85. Conditions to be satisfied by Masonry Arches, Abutments, etc....... II7

§ 2. MASONRY ARCH. LINE OF PRESSURE.

86. Definitions................................ I18

87. Line of Pressure a Funicular Polygon................... I18

88. A Test of Stability .............................. I19

89. Relation between Line of Pressure and Form of Arch-ring.......... I22

9o. Maximum and Minimum Crown Pressure.................. 124

91. Location of True Line of Pressure.................... 124

92. Example.................................... 129

93. Unsymmetrical Cases............................. 131

§ 3. ABUTMENTS, PIERS, ETC.

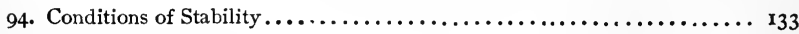

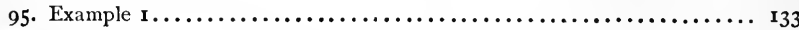

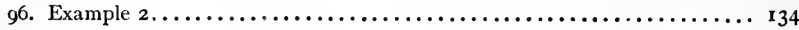

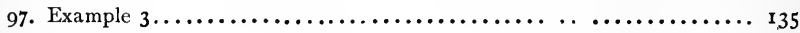

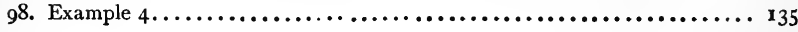




\section{GRAPHIC STATICS.}

CHAPTER I.

GENERAL METHODS.

§. Introduction.

I. Graphic Statics has for its object the solution of problems in statics by means of geometrical constructions, the results being obtained directly from the scale drawings.

(A knowledge of Statics is presupposed. However, a brief presentation of its principles and methods in case of forces lying in the same plane will be made.

In the subsequent chapters, familiarity with the subject of Strength of Materials, including beam stresses and deflections, is also assumed.)

2. Representation of Forces. A force is fully determined when its magnitude, direction, and point of application are known. In dealing with problems in Statics of Rigid Bodies, the magnitude, direction, and line of action of a force are the elements commonly involved, since the equilibrium or motion of such a body is not affected by transferring the point of application of a force to any other point in its line of action.

3. Resultant of any System of Forces Lying in the Same Plane. The magnitude and direction of the resultant of 
any system of forces lying in the same plane may be found by either of the following methods:

I. Geometrically. Represent the given forces by the sides of a polygon taken in order. The closing side in reverse order is the resultant in magnitude and direction.

2. Algebraically. Resolve each force $F$ into components $F_{x}$ and $F_{y}$, parallel to coordinate axes $X$ and $Y$ respectively. Then $R_{x}=\Sigma F_{x}$ and $R_{y}=\Sigma F_{y}$. Combining these we have

$$
\begin{aligned}
& R=\sqrt{R_{x}^{2}+R_{y}^{2}}, . . \\
& \tan \alpha_{r}=\frac{R_{y}}{R_{x}} \text {. . . . . . . }
\end{aligned}
$$

Equations (I) and (2) give the magnitude and direction of the resultant, $R$ being its magnitude and $\alpha_{r}$ the angle it makes with $X$.

The line of action of the resultant may be found by either of the following methods:

I $a$. Geometrically. Combine the forces in succession by means of the principle that the resultant of two forces lying in the same plane must pass through their point of intersection.

2a. Algebraically. Use the method of moments, i.e., The moment of the resultant of any such system of forces, about any axis perpendicular to the plane of the forces, is equal to the algebraic sum of the moments of the forces.

In case the magnitude of the resultant is zero, the forces either form a couple or are in equilibrium. If the resultant is a couple, its moment can be found by $2 a$ or the given forces can be combined into a single resultant couple by $\mathrm{\text {I }}$. Equilibrium is the special case of a couple whose moment is zero.

If the lines of action of the given forces intersect at a common point, the line of action of the resultant will pass through this point, its magnitude and direction being found by the methods previously stated. 
It will be observed that the determination of the resultant, when it is a single force and not a couple, involves two operations, namely: I. The determination of its magnitude and direction. 2. The determination of its line of action. We may perform both of these operations graphically by means of $\mathrm{I}$ and $\mathrm{I} a$, or algebraically by means of 2 and $2 a$.

4. Example. Find the resultant of the four forces $F, F^{\prime}, F^{\prime \prime}$, $F^{\prime \prime \prime}$ (Fig. I A) by each of the preceding methods.

First Solution. Represent the given forces by the sides of the polygon (Fig. I B) taken in order; then the closing side, $A D$, represents the magnitude and direction of the resultant. The numerical values of these quantities may be found (I) by solving the polygon by trigonometry, or (2) by

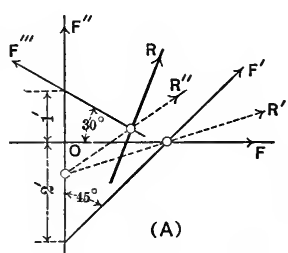

FIG. I.

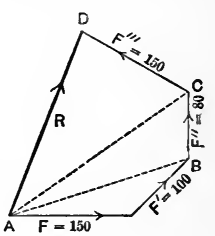

(B) direct measurement from a scale drawing.

To find the line of action of the resultant by the first method, we can proceed as follows: The magnitude and direction of the resultant of $F$ and $F^{\prime}$ is $A B$. Its line of action, $R^{\prime}$, is drawn parallel to $A B$ through the intersection of the lines of action of $F$ and $F^{\prime}$. Continuing in the same manner, we determine $R^{\prime \prime}$ to be the line of action of the resultant of $F, F^{\prime}$, and $F^{\prime \prime}$, and finally $R$ to be the line of action of the resultant of the four forces.

Second Solution. Resolve each of the four forces into horizontal $(H)$ and vertical $(V)$ components. Then

$$
\begin{aligned}
& \Sigma H=\mathrm{I} 50+100 \cos 45^{\circ}+0-150 \cos 30^{\circ}=90.8, \\
& \Sigma V=0+100 \sin 45^{\circ}+80+150 \sin 30^{\circ}=225.7,
\end{aligned}
$$




$$
\begin{aligned}
& R=\sqrt{(\Sigma H)^{2}+(\Sigma V)^{2}}=243 \cdot 3, \\
& a_{r}=\cos ^{-1}\left(\frac{\Sigma H}{R}\right)=68^{\circ} 5^{\prime} .
\end{aligned}
$$

To find the line of action of $R$, apply the method of moments. Using $O$ as moment axis, we have

$$
\Sigma M=\mathrm{I} 50 \cdot 0-\mathrm{I00} \cdot \mathrm{I} \cdot 4 \mathrm{I} 4+80 \cdot 0-\mathrm{x} 50 \cdot .866=-27 \mathrm{I} \cdot 3 .
$$

Hence the moment of the resultant about $O$ is left-handed, and its distance from $O$ is $\frac{\Sigma M}{R}=$ I.II5. This locates the resultant as given in Fig. $\mathrm{x}$.

The moment arms of the several forces may be computed or may be measured directly from a scale drawing when the results thus obtained are sufficiently exact.

Problem. Represent four forces by the sides of a closed polygon taken in order. Assume the lines of action of these forces at random. Find the moment of the resultant couple by each of the two methods of Art. 3 .

5. Conditions of Equilibrium. Forces not acting at the same point. The conditions of equilibrium may be deduced from the fact that any system of balanced forces can be reduced to two equal and opposite forces having the same lines of action, i.e., a couple whose moment is zero.

A. If the resultant is a couple, $R=0$, hence:

I. Geometrically. The given forces can be represented in magnitude and direction by the sides of a closed polygon taken in order.

2. Algebraically. If the forces be resolved into components parallel to coordinate axes, the algebraic sum of each set of components must be zero, i.e., $\Sigma F_{x}=0$ and $\Sigma F_{y}=0$. 
B. In order for the moment of the resultant couple to be zero, we have:

Ia. Geometrically. The line of action of the resultant of any portion of the given forces must coincide with the line of action of the resultant of the remainder.

2a. Algebraically. The algebraic sum of the moments of the forces must be zero.

I and $\mathrm{I} a$ constitute the geometrical, and 2 and $2 a$ the algebraic conditions of equilibrium.

Forces acting at the same point. In this case condition 1 or 2 is sufficient. The condition that if three non-parallel forces balance they must intersect at a common point, is a special case under $\mathrm{I} a$.

When any system of forces lying in the same plane is in equilibrium, one or more of the preceding conditions of equilibrium serve to determine the unknown elements of the problem, if it is solvable under the assumption that the body acted on is rigid.

6. Examples. I. The truss (Fig. 2) is in equilibrium under the supporting forces $P$ and $P^{\prime}$ and the load $W . \quad P$ is vertical and $W$, the known force, is normal to the roof surface at its middle point $O$. Indicate how to find $P$ and $P^{\prime}$ by each of the following methods (see Art. 5): (I) By using r $a$ and $\mathrm{r}$; (2) by using $2 a$ alone; (3) by using $2 a$ to find one force, then $\mathrm{I}$ to find the remaining force; (4) by using $2 a$ to find one force, then 2 to find the remaining force.

First Solution. The resultant of $P$ and $W$, acting through $N$, must balance the remaining force, $P^{\prime}$, acting at $M$; hence the line of action of $P^{\prime}$ must be $M N$. The forces $P, W$, and $P^{\prime}$ to balance must form a triangle (not shown), the sides taken in order. Plotting $W$ and completing the triangle, $P$ and $P^{\prime}$ are determined.

Second Solution. Substitute for $P^{\prime}$, unknown in magnitude 
and direction, the two component forces $P_{H}^{\prime}$ and $P_{V}^{\prime}$, unknown in magnitude only. Also, for convenience, substitute for $W$ its $H$ and $V$ components. To find $P$ take the moment axis at $M$, the intersection of the two other unknown forces. Then the algebraic sum of the moments of $P, W_{H}, W_{V}, P_{H}^{\prime}$ and $P_{V}^{\prime}$ about $M$ must be zero. Solving the equation thus formed, we determine

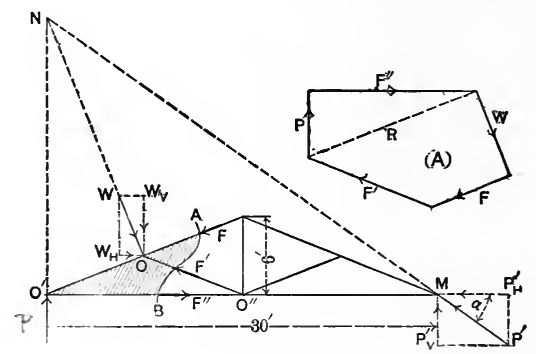

FIG. 2 .

$P$. Similarly, to find $P_{V}^{\prime}$, take moments about $O^{\prime}$. To find $P_{H}^{\prime}$ take moments about any convenient point not on its line of action, e.g., $O$.

Third Solution. Find one force, e.g., $P$, by moments, then plotting $P$ and $W$, the closing side of the triangle formed gives the magnitude and direction of $P^{\prime}$.

Fourth Solution. Find one force, as $P$, by moments. Then, since $\Sigma F_{x}$ and $\Sigma F_{y}$ must each be zero, we have

$$
\begin{aligned}
& P_{H}^{\prime}=W_{H} \text { and } P_{V}^{\prime}=W_{V}-P . \\
& P^{\prime}=\sqrt{ }\left(P_{H}^{\prime}\right)^{2}+\left(P_{V}^{\prime}\right)^{2} \text { and } \tan \alpha=\frac{P_{V}^{\prime}}{P_{H}^{\prime}} .
\end{aligned}
$$

Example 2. The portion of the truss (Fig. 2) to the left of $A B$ is in equilibrium under the action of the supporting force $P$, 
load $W$, and the forces $F, F^{\prime}$, and $F^{\prime \prime}$ exerted by the portion of the truss to the right of the section $A B$ upon the left-hand portion. The lines of action of these latter forces coincide with the centre lines of the members cut by $A B$, and their magnitudes are equal to the stresses existing in these members. $\quad P$ and $W$ being known, indicate how to find the three unknown forces by each of the following methods (see Art. 5):

(I) By using conditions I $a$ and I; (2) by using $2 a$ alone; (3) by using $2 a$ to find one force, then I to find the remaining two; (4) by using $2 a$ to find one force, then 2 to find the remaining two forces.

First Solution. The resultant of $P$ and $F^{\prime \prime}$ acting through $O^{\prime}$ must balance the resultant of $W, F$, and $F^{\prime}$ acting through $O$. Hence the line of action of each resultant, $R$, must be $O O^{\prime}$. $-P$, $F^{\prime \prime}$, and their resultant $R$, must form a triangle (Fig. $2 \mathrm{~A}$ ); $P$ being the known force, $F^{\prime \prime}$ and $R$ are thus determined. The resultant, $R$, must balance the forces at $O$, hence $R, W, F$, and $F^{\prime}$ must form a closed polygon as shown in Fig. 2 A. $F$ and $F^{\prime}$, the remaining unknown forces, are thus determined by plotting $W$ and completing the polygon.

Second Solution. To find $F$, take the moment axis at the intersection $O^{\prime \prime}$ of the other two unknown forces, so that their moments will each be zero. Then the algebraic sum of the moments of $P, W$, and $F$ about $O^{\prime \prime}$ must equal zero. Solving the equation thus formed, we determine $F$. Similarly, to find $F^{\prime}$ take moments about $O^{\prime}$, and to find $F^{\prime \prime}$ take moments about $O$.

Third Solution. We find one force, as $F$, by the preceding method, then represent the known forces $P, W, F$, by the sides of a polygon taken in order, and complete the polygon by lines parallel to the two remaining forces, $F^{\prime}$ and $F^{\prime \prime}$.

Fourth Solution. We find one force, $F$, by the method of moments as before. Then, placing the algebraic sums of the hori- 
zontal and vertical components of the forces each equal to zero, we form two equations which are solved for the two remaining unknown forces, $F^{\prime}$ and $F^{\prime \prime}$.

In algebraic solutions the directions of the unknown forces are assumed, the algebraic signs of the results indicating whether the assumed directions are correct or not.

Problem I. In Example I, obtain the numerical results, solving by each of the four methods mentioned. $W=5000 \mathrm{lbs}$.

Problem 2. In Example 2, obtain the numerical results, solving by each of the four methods mentioned. Also determine the kind of stress (tension or compression) in the members cut by $A B$.

Remarks. The student should become familiar with each mode of solution so as to be able to select readily that one which is best adapted to the problem at hand.

Ability to make such selection can be secured only by solving a variety of problems by various methods and comparing the merits of the different solutions.

In using the method of moments, it should be noted that it is usually better to use the $H$ and $V$ components of the forces than it is to use the original forces.

It should also be noted that in case of forces not acting at the same point but lying in the same plane, three unknown quantities can be determined, while if the forces act at the same point only two can be determined.

\section{§. Funicular Polygon.}

7. Definitions. Let $F, F^{\prime}, F^{\prime \prime}$ (Fig. $3 \mathrm{~A}$ ), be given forces, their magnitudes being represented by $A B, B C, C D$ (Fig. 3 B). Assume any point $P$, and draw the radial lines $P A, P B$, etc. From any point $M$ on the line of action of $F$ draw $M L$ and $M N$ parallel 
to $P A$ and $P B$ respectively. From $N$, where $M N$ intersects the line of action of $F^{\prime}$, draw $N O$ parallel to $P C$; similarly draw $O Q$ parallel to $\mathbf{L}^{\prime}$ $P D$, thus forming the broken line $L M N O Q$. We may consider $A P$ and $P B$, having

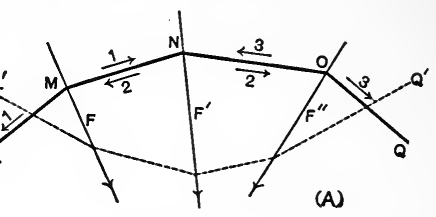
the directions of the arrows marked 1 , to be the components of the force $F$, the lines of action of these components being $M L$ and $M N$ respectively. Similarly, $B P$ and $P C$, having the directions marked 2 and the

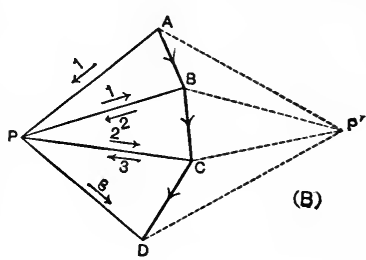

Frg. 3. lines of action $N M$ and $N O$, may be taken as components of $F^{\prime}$; and $C P$ and $P D$ with $O N$ and $O Q$ for lines of action as the components of $F^{\prime \prime}$. $M N$ is thus the line of action of two equal and opposite forces $P B$, and $N O$ of the two equal and opposite forces $P C$. These two pairs of forces consequently balance, leaving $A P$ and $P D$, having $M L$ and $O Q$ for their lines of action, as the equivalent of the original forces.

The broken line LMNOQ is called a funicular or equilibrium polygon. The former name is given because the line corresponds to the shape assumed by a weightless cord when fastened at the ends and acted on by the given forces. This is shown by the polygon drawn in dotted lines. The latter name is applicable since a jointed frame of the form of the polygon would be in equilibrium under the action of the given forces.

The point $P$ is called the pole; the lines $P A, P B$, etc., are the rays; and the corresponding lines of the funicular polygon are its strings. 
Figure $3 \mathrm{~A}$ is called the space diagram, since it represents the location of the lines of action of the forces. Its scale is one of distance. Figure $3 \mathrm{~B}$ is the force diagram, the lengths of its lines representing the magnitudes of the forces to scale. The perpendicular distance from the pole to any side of the force polygon is called the pole distance of that force. It is to be noted that this distance represents a force magnitude.

In the case of parallel forces the force polygon becomes a straight line, and the pole distances of all the forces are equal.

8. Applications. The following results are readily derived from the construction explained in Art. 7 .

(I) The resultant of $F, F^{\prime}$, and $F^{\prime \prime}$ is given in magnitude and direction by the closing side $A D$ of the force polygon, and its line of action passes through the point of intersection of the strings $L M$ and $O Q$. The resultant is thus completely determined; and, in general, the line of action of the resultant of any system of forces, taken consecutively, passes through the point of intersection of the two strings between which the forces lie.

(2) If the force polygon is closed, $P A$ and $P D$ will coincide, and hence the corresponding strings $I M$ and $O Q$ will be parallel. In this case the resultant is a couple whose arm is the perpendicular distance between the parallel strings, and whose forces are represented by the ray $(P A=P D)$ corresponding to these strings.

(3) In order for the given forces to be in equilibrium, the arm of the couple in (2) must be zero; that is, the strings $L M$ and $O Q$ must coincide in $M O$. In this case, the funicular polygon is said to be closed. The conditions of equilibrium, therefore, are that both the force and funicular polygons must close.

If the forces intersect at a common point, the first of these conditions is sufficient, since such a set of forces cannot form a couple. 
(4) Any number of funicular polygons may be drawn for the same system of forces by using different poles and beginning the construction of the polygons at different points. Various geometrical relations exist between these different polygons, some of which are given in $\S 5$. The following relations are derived directly from the preceding discussion.

(a) Corresponding pairs of non-parallel strings of the various funicular polygons must intersect on the same straight line, this being the line of action of the resultant of the forces included by these strings.

(b) In the case where the force polygon closes, by using different poles different couples will be obtained; but as these couples are all equally the resultarit of the given system of forces, their moments will be equal.

It is obvious that any string can be given any desired direction by drawing the corresponding ray in this direction and assuming, the pole at any point on the line thus drawn.

In selecting the pole, the obtaining of accurate and convenient diagrams is kept in view. Generally the rays should not make very oblique angles with the adjacent lines of the force polygon. If the pole is taken at a vertex of the force polygon, as at $A$, Fig. $3 \mathrm{~B}$, the construction of the funicular polygon becomes identical with that explained in Art. 4, for finding the line of action of the resultant by $\mathrm{I} a$. The construction in Art. 4 frequently leads to inaccurate and inconvenient diagrams and is inapplicable to parallel forces.

9. Notation. Resultant of a System of Forces. To illustrate the notation to be used, let it be required to find the resultant of the four forces $A B, B C, C D, D E$ (Fig. 4). The line of action of any force is designated by the two letters between which it lies; thus, $a b$ represents the line of action of the first force, $b c$ of the second 
force, etc. In the force diagram, the same letters in capital type are used, but placed at the ends of the lines representing the

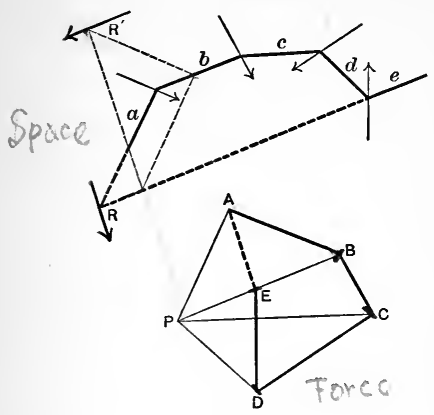

FIG. 4 . forces. In constructing the force polygon, the forces and letters must be taken in order from the space diagram (e.g., proceeding from left to right): When this is done, if the letters are read in order from left to right in the space diagram, the same order of letters in the force polygon is used to indicate the directions in which the successive forces act. It will be noticed that the string corresponding to the ray $P A$ lies in the space $a$, that corresponding to $P B$ in the space $b$, etc. These strings will be referred to as the strings $a, b$, etc., and the corresponding rays as the rays $A, B$, etc.

Having constructed the force and funicular polygons, the magnitude and direction of the resultant is represented by the closing side, $A E$, of the force polygon, and one point in its line of action is the point of intersection of the similarly lettered strings ( $a$ and $e$ ). The line of action of the resultant, $R$, is then drawn through this point of intersection, parallel to $A E$. In referring to any force (e.g., the force $B C$ ), the capital letters will be employed, although the line of action of the force as well as its magnitude and direction is included in the reference.

The system of notation just described is applicable in most cases occurring in engineering practice. When the location of the forces is such that it cannot be used to advantage, the modified notation illustrated in Fig. 5 may be employed instead. This needs no description. 
In general, in referring to forces in the text, the letters will be used so as to indicate by their order of succession the direction in which the forces act.

Special Case. In finding the resultant of a system of forces the case may occur in which the two strings whose intersection locates the resultant are parallel (but do not form a couple), or are so nearly parallel that they do not intersect conveniently. In

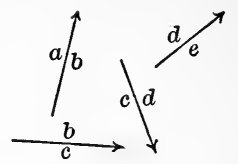
such cases the resultant may be located as follows: Let it be required to find the resultant of the forces $B C, C D$, and $D E$ (Fig. 4). This resultant is $B E$, its line of action passing through the intersection of the strings $b$ and $e$, which here are parallel. Now we know (Art. 7) that the forces under consideration are equivalent to the two forces represented by the end strings $b$ and $e$ and the corresponding rays, $B P$ and $P E$. Hence we can solve the problem by finding the resultant of these two forces by means of a second funicular polygon, as follows: Taking the point $A$ as a convenient pole, draw a funicular polygon (shown in dotted lines) for the forces $B P$ and $P E$. The intersection of the end strings at $R^{\prime}$ lucates the resultant which is drawn parallel to $B E$.

\section{Problems.}

(I) Assume five non-parallel forces, and find their resultant, using two different poles.

(2) Assume five non-parallel forces whose force polygon closes, and find their resultant, using two different poles.

(3) Assume five parallel forces and find their resultant, using two different poles.

(4) Assume five parallel forces whose algebraic sum is zero and find their resultant, using two different poles. 
II. Distributed Forces. Distributed forces are commonly represented in terms of their intensity. The intensity of a distributed force is the force per unit length, area, or volume, as the case may be. Gravity and water pressure are familiar illustrations of such forces.

If the intensity is the same at all points, the force is uniformly distributed.

Parallel forces lying in the same plane will alone be consid-

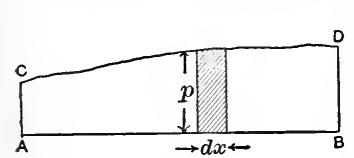

FIG. 6. ered here. Such forces are represented as follows:

Let $A B$ (Fig. 6) be any line perpendicular to the direction of the forces, $d x$ any infinitesimal portion of $A B$, and let the ordinate, length $p$, represent the intensity of the force at the point from which the ordinate is drawn. The shaded area $p d x$ thus represents the amount of force acting on the length $d x$ of the line. The diagram (Fig. 6) is then to be interpreted as follows:

The length of the ordinate at any point of the line $A B$ represents the intensity of the force at that point, and the area included between any two ordinates represents the amount of force acting on the corresponding portion of $A B$.

I2. Funicular Polygon for a Distributed Force. Let $W X Y Z$ (Fig. 7) represent a distributed force. Divide $X Z$ into equal divisions sufficiently small so that the force represented by any area, as $S T U V$, may be taken to act at the middle point $n$ of the division and the area may be taken to be equal to $U V \times m n$. Then the distributed force is equivalent to the four forces $A B$, $B C, C D$, and $D E$, their magnitudes $(A B, B C$, etc., of the force polygon) being proportional to the lengths of the corresponding middle ordinates, such as $m n$. The funicular polygon $G H$ is 
drawn from this system of forces. Now it is evident that as the divisions of $X Z$ are made smaller, this polygon would more closely approach the true funicular polygon (curve) for the distributed force. The true funicular polygon would then be a curve tangent to the polygon $G H$ at the points (marked with short lines) corresponding to the points of division of the distributed
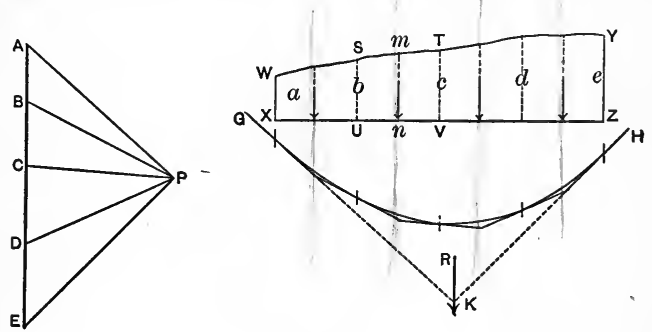

FIG. 7 .

force. Each string would be infinitesimal in length and have the direction of a tangent to the curve.

Such a funicular polygon can be used for the same purposes and in a similar manner to one drawn for concentrated forces. Thus to find the line of action, $R$, of the resultant force $A E$, we would draw tangents, $G K$ and $H K$, to the curve at the points corresponding to $X$ and $Z$, these tangents being drawn parallel to the rays $A$ and $E$ of the force diagram.

I3. Funicular Polygon for a Uniformly Distributed Force. A familiar construction of analytical geometry for a parabola with axis vertical is shown in Fig. 8. It consists in dividing a vertical line $\mathrm{I}-8$ into equal parts and drawing a series of equidistant vertical lines as shown. The broken line $I^{\prime}-8^{\prime}$ is then obtained by drawing its successive segments, $\mathbf{r}^{\prime}, \mathbf{2}^{\prime}$, etc., parallel to $\mathrm{Pr}_{\mathrm{r}}$, 
$P_{2}$, etc. A curve tangent to this broken line at the middle points of its segments is a parabola with axis vertical.

Comparing this construction with that of Fig. 7 , we see that it is identical with the construction of a funicular polygon for a.

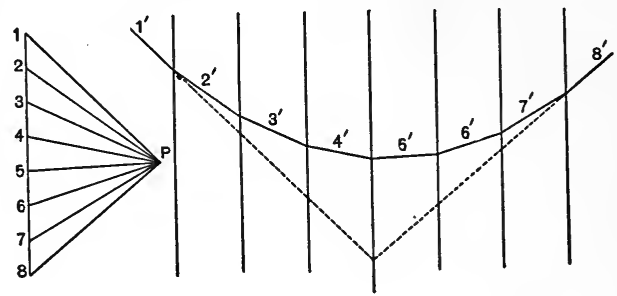

Fig. 8.

uniformly distributed force. The funicular polygon for a uniformly distributed force is thus a parabola whose axis is parallel to the force.

If, then, the force $W X Y Z$ (Fig. 7) were uniformly distributed,

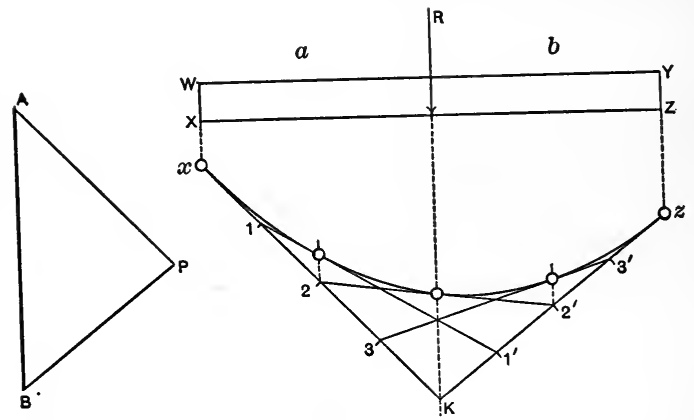

FIG. 9.

the funicular curve would be a parabola, axis vertical, tangent to the strings $K G$ and $K H$ at the points corresponding to 
the limits of the force. Such a parabola can be constructed as follows (see Fig. 9):

Plot the resultant force $A B$, its line of action $R$ bisecting $X Z$, and draw the strings $K x$ and $K z$ parallel to the rays $A$ and $B$ respectively. Divide $K x$ and $K z$ into the same number of equal parts, as at I, 2, 3 and $\mathrm{I}^{\prime}, 2^{\prime}$ and $3^{\prime}$. Lines joining these points of division, as shown, are tangents to the required curve. The points of tangency, indicated by circles, are located by drawing lines parallel to $R$ through the alternate points of division (compare with Fig. 8).

\section{§. Forces in Equilibrium.}

14. Use of Funicular Polygon. It will be noticed from $\S 2$ that the funicular polygon construction is especially adapted to the case of parallel and other non-concurrent forces, although it may be used to advantage in problems relating to forces intersecting at a common point, when this point lies outside the limits of the drawing. The conditions of equilibrium, given in Art. 8, for non-concurrent forces are (I) that the force polygon must close, and (2) that the funicular polygon must close. The application of these conditions to the solution of problems will now be illustrated, the cases most frequently arising in practice being presented.

15. Case I. Parallel Forces. Given a system of parallel forces in equilibrium, the lines of action of all and the magnitudes and directions of all but two being known. It is required to find the unknown elements.

Let the unknown forces be the supporting forces of the beam (Fig. Io). Represent the loads in succession by the sides $A B$, $B C, C D$ of the force polygon, and, selecting a suitable pole, draw 
the strings $a, b, c, d$ of the funicular polygon. The string $a$ intersects the left reaction at $m$, and the string $d$ the right reaction at $n$.

The line joining $m, n$ is then the closing string $e$ of the funicular polygon, and the ray $P E$ drawn parallel to it determines $D E$ and

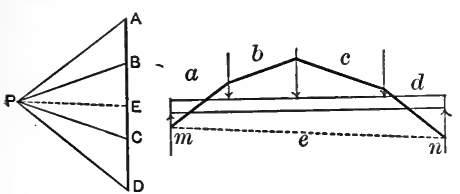

FIG. Io. $E A$ to be the right- and lefthand supporting forces respectively. When the unknown (supporting) forces are parallel, but the given forces (loads) are not, the supporting forces must be parallel to the resultant load. If we take $A D$ (Fig. 10) to represent this resultant load, the construction for determining the magnitudes of the supporting forces will be similar to that just explained.

The method of determining the magnitudes and directions of the supporting forces from the lettering, after the construction is completed, should be carefully noted. It is as follows:

(I) The strings are lettered with the same letters as the corresponding rays, so that the strings intersecting on the line of action of any force have the same letters as those which represent that force in the force polygon. The two strings intersecting on the left supporting force are $a$ and $e$, hence $A E$ represents the magnitude of this force. Similarly $d$ and $e$ intersect on the right supporting force; so its magnitude is $D E$.

(2) The forces are laid off in the force polygon in right-handed order; i.e., if the letters representing them in the space diagram be read in right-handed order, the same order of letters in the force polygon indicates the direction in which the forces act. We also know that the force polygon must close. The force polygon then is $A B C D E A$, the order of letters $D E, E A$ indicating that each supporting force acts upwards. It should be noted in this 
and the following solutions that the known forces are made to follow consecutively in constructing the force polygon.

(To obtain a thorough understanding of these solutions, it would be well for the student to trace out the construction from the standpoint of the triangle of forces. Thus, in the present case, the force $A B$ is resolved into $A P$ and $P B$, whose lines of action are the strings $a$ and $b$. Similarly $B C$ is resolved into $B P$ and $P C$, and $C D$ into $C P$ and $P D$. The two forces $P B$ and $B P$ balance since they are equal and opposite and have the same line of action, $b . \quad P C$ and $C P$ balance for a similar reason; hence the three loads are equivalent to $A P$ and $P D$, whose lines of action are $a$ and $d$ respectively. Now, in order to have equilibrium, the resultant of $A P$ and the left supporting force must balance the resultant of $P D$ and the right supporting force. The lines of action of these two resultants must then coincide in $m n$. Since, of the three forces intersecting at $m$, one is the resultant of the other two, they must form a triangle, $A P$ being the known magnitude. This triangle is $A P E$; thus the reaction $E A$ is determined. The corresponding triangle for the forces intersecting in $n$ is $E P D$.)

I6. Case II. Non-parallel Forces. Given a system of forces in equilibrium, all but two of which are known completely. Of these two, the line of action of one and one point in the line of action of the other are known. It is required to determine the unknown elements.

Let the known forces be the wind pressures $A B, B C, C D$ on the roof (Fig. II). The unknown forces are the reactions of the supports. The line of action of the right supporting force is given, it being vertical; and one point, $m$, of the left reaction is known. Represent the loads in succession by the sides $A B, B C, C D$ of the force polygon. The strings $e$ and $a$ must intersect on the 
line of action of the supporting force $E A$, and as the point $m$ of this line of action is known, the funicular polygon will be constructed so that these strings will intersect at this point. The construction of the funicular polygon is then begun by drawing
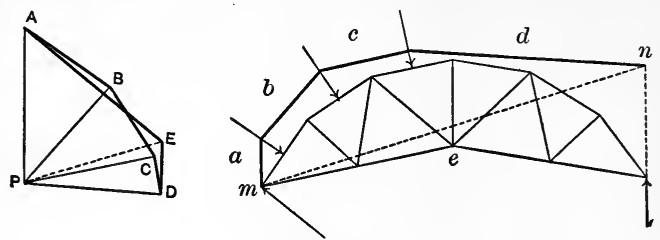

FIG. II.

the string $a$ through the point $m$, the strings $b, c, d$ being then drawn in order.

The string $d$ intersects the right supporting force at $n$, and, of course, the string $a$ intersects the left supporting force at $m ; m n$ is thus the closing side, $e$, of the funicular polygon. The ray corresponding to the string $e$ is now drawn, intersecting the right supporting force, $D E$, at $E$. The triangle of forces, $P D E$, whose lines of action intersect at $n$, is thus formed, and the magnitude of the supporting force $D E$ is determined. Finally, the closing side, $E A$, of the force polygon represents the other supporting force in magnitude and direction. Its line of action is drawn through $m$, parallel to $E A$.

17. Case III. Non-parallel Forces. Given a system of forces in equilibrium, the lines of action of all are known, but the magnitudes and directions of three of the forces
are unknown. It is required to determine the un-
known elements. Let $a b, b c, c d$ (Fig I2) be the

Fig. 12. lines of action of the three unknown forces. The resultant of any two, as $b c$ and $c d$, must pass through their point 
of intersection, $n$. If this resultant be substituted for $b c$ and $c d$, the problem becomes identical with Case II; $a b$ being the line of action of one unknown force, and $n$ one point of the line of action of the other.

The construction of Case II having been made, the resultant of $b c$ and $c d$ can be resolved into components parallel to these lines of action, thus completing the solution. Figure I 3 shows this
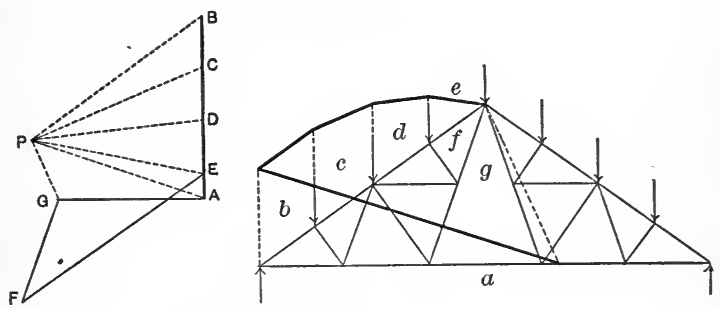

FIG. 13.

method applied to determine the stresses in the members $e f, f g$, and $g a$, of the truss, the loads and reactions being known.

I8. Resolution of Forces into Components. Since forces equal and opposite to the components and having the same lines of action will balance the given forces, we can solve problems of this nature in the same manner as if the forces balanced, the desired components being the balancing forces with their directions reversed.

§ 4. Graphical Determination of Moments.

r9. Moment of Resultant of any System of Forces. The resultant of $A B, B C$ and $C D$ (Fig. I4) is $A D$, its line of action, 
lettered $R$, passing through th 2 intersection of the strings

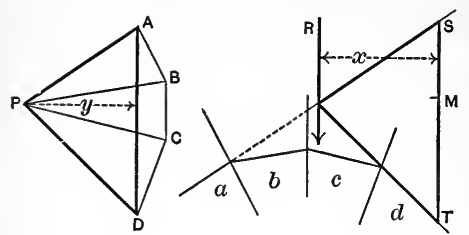

FIG. I4. $a$ and $d$. The moment of this resultant about any point $M$ is thus $(R=A D) x$, in which $x$ is the moment arm. Now draw $S T$ through $M$ parallel to $A D$ and terminating in the strings $a$ and $d$, thus forming with these strings a triangle (drawn in heavy lines) similar to $P A D$. From these two similar triangles we have, $A D \cdot x=S T \cdot y$. But $A D \cdot x$ is the moment of the resultant force, hence

The moment of the resultant of any system of forces may be found by drawing a line parallel to the resultant through the moment axis, noting the distance intercepted on this line by the strings corresponding to the resultant. The product of this intercept into the pole distance of the resultant is the desired moment. This method has no value except in case of parallel forces.

20. Moment of Resultant of Parallel Forces. The constructions of this section are especially adapted to the case of parallel forces. Illustration: Let the beam (Fig. I5) be loaded as shown. The reactions of the supports (Art. I5) are found to be $C D$ and $D A$. It is required to find the bending moment at any section, $S T$. By definition, this bending moment is equal to the algebraic sum of the moments of the forces to the left of the section, and, by Art. I9, this resultant moment is equal to the pole distance, $y$, times the intercept $S T$.

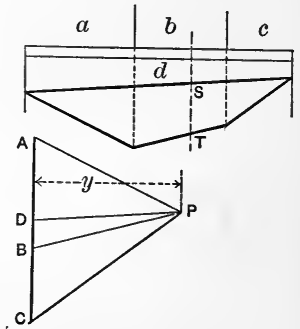

FIG. I5. 
Now in case of parallel forces, the pole distance is the same for all the forces, hence the bending moments at the various sections of the beam are directly proportional to the corresponding intercepts. This subject is further developed in Chapter III.

It follows directly from Arts. I9 and 20 that if any two funicular polygons be constructed for a given system of parallel forces, the ratio of the intercepts of corresponding pairs of strings on any line drawn parallel to the forces is constant, being equal to the inverse ratio of the pole distances of the respective polygons.

\section{§. Some Special Constructions for Funicular Polygons.}

21. Locus of Points of Intersection of Corresponding Strings.

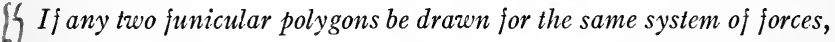
their corresponding strings will intersect on a straight line parallel to the line joining the two poles.

Proof. Let $A B, B C, C D$ (Fig. I6) be the given forces. (The student should keep both diagrams. in mind, also that the order of letters in the text indicates the directions of the forces.) The funicular polygons for the two poles, $P$ and $P^{\prime}$, are drawn in full and broken lines respectively.

The resultant of $P A$ and $A P^{\prime}$ is $P P^{\prime}$. Its line of action $x z$ is parallel to $P P^{\prime}$ and

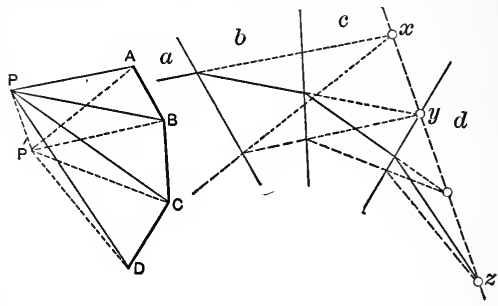

Fig. I6. passes through $x$, the point of intersection of the strings $a$ and $a^{\prime}$ which are the lines of action of the forces $P A$ and $A P^{\prime}$ respectively. Similarly, the resultant of $P B$ and $B P^{\prime}$ is $P P^{\prime}$, its line of action $y z$ passing through the intersection of the strings $b$ and $b^{\prime}$, etc. 
Now $P B$ is the resultant of $P A$ and $A B$, also $B P^{\prime}$ is the resultant of $B A$ and $A P^{\prime}$. Since $A B$ and $B A$ are balanced forces, the resultant of $P B$ and $B P^{\prime}$ is the same as the resultant of $P A$ and $A P^{\prime}$; hence their lines of action, $x z$ and $y z$, must coincide. The same reasoning applies to the intersections of the remaining strings. The points $x, y$, etc., therefore lie on the line $x z$, parallel to $P P^{\prime}$, this being the line of action of the common resultant of the pairs of forces, $P A$ and $A P^{\prime} ; P B$ and $B P^{\prime} ; P C$ and $C P^{\prime}$, etc.

\section{Locus of Poles of Funicular Polygons passing through} Two Given Points. The locus of the poles of all funicular polygons, two of whose corresponding strings pass through two given points, is a straight line parallel to the line joining the two points.

Proof. Let $A B, B C, C D$ (Fig. I7) be the given forces and $x$ and $y$ be the points through which the strings $a$ and $d$ respectively
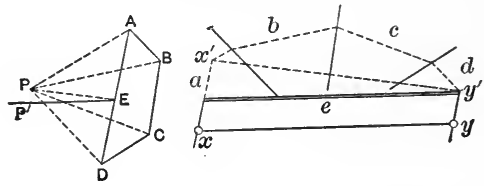

FIG. I7.

are to pass. Assume two balancing forces $D E$ and $E A$, parallel to $A D$ and passing through the points $y$ and $x$ respectively. The magnitudes of these balancing forces are found, as in Art. 15, by drawing the closing string $e\left(x^{\prime} y^{\prime}\right)$ of the funicular polygon and the corresponding ray $E$. Now since the forces $A B C D E A$ are balanced, all funicular polygons drawn for them will close. If, then, we wish the string $a$ of any such polygon to pass through $x$ and the string $d$ through $y$, the string $e$ must evidently pass through both $x$ and $y$, and the pole $P^{\prime}$ therefore lie somewhere on the ray drawn through $E$ parallel to $x y$, i.e., parallel to the line joining the two given points. 
23. Funicular Polygon through Three Points. Problem. To draw a funicular polygon for a given system of forces, such that three designated strings shall pass through three given points. Let the forces be $A B C D E F$ (Fig. I8). It is required to draw a funicular polygon such that the string $a$ will pass through $O$, the string $c$ through $O^{\prime}$, and the string $f$ through $O^{\prime \prime}$.
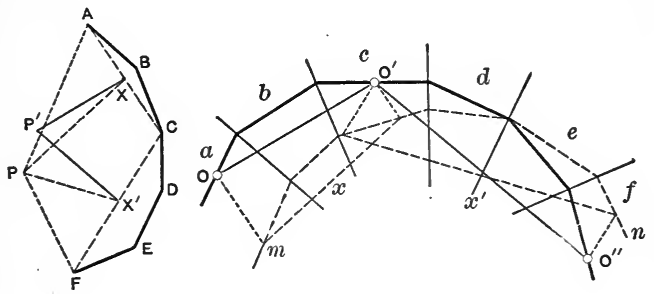

FIG. I8.

Let $m n$ be any funicular polygon for the given forces, with $P$ for pole. By means of the construction of Art. 22, we determine $P^{\prime} X$ to be the locus of the poles of all funicular polygons whose strings $a$ and $c$ pass through $O$ and $O^{\prime}$ respectively. Also, by the same construction we determine $P^{\prime} X^{\prime}$ to be the locus of the poles of all funicular polygons whose strings $c$ and $f$ pass through $O^{\prime}$ and $O^{\prime \prime}$ respectively. Hence, in order for both conditions to be satisfied, the pole must lie on both $P^{\prime} X$ and $P^{\prime} X^{\prime}$, i.e., at their point of intersection, $P^{\prime}$. The required polygon is drawn in full lines.

(Note. To secure accuracy, draw the strings $a, c$, and $f$ first, then draw the intermediate strings closing on the ones midway between the given points.)

24. Funicular Polygon through Three Points. Parallel Forces. A shorter solution than that given in Art. 23 can be made for this case. Let $A B, B C, C D$, and $D E$ (Fig. I9) be the given forces, it 
being required to draw a funicular polygon for them such that the string $a$ will pass through $O$, the string $c$ through $O^{\prime}$, and the string $e$ through $O^{\prime \prime}$. Let $m n$ be any funicular polygon for the given forces, $P$ being the pole. Draw lines parallel to the forces through each of the three given points. Taking the ones through $O$ and $O^{\prime \prime}$ to be the lines of action of balancing forces, and following the construction of Art. 22, we determine $m n$ to be the closing string $(f)$ of the polygon drawn, the closing string of the required
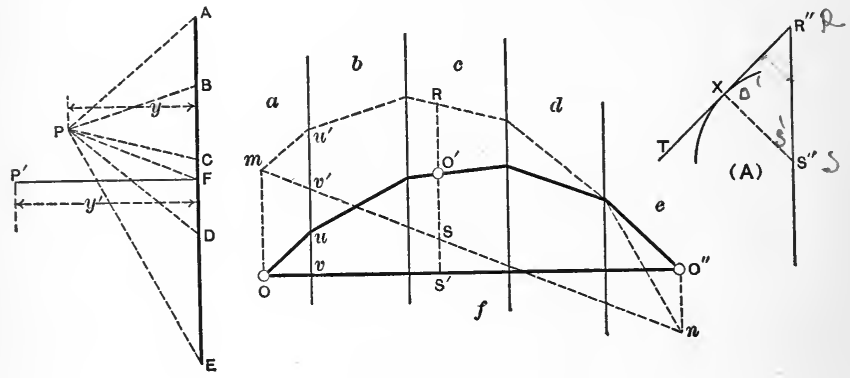

FIG. I9.

polygon being $O O^{\prime \prime}$. Knowing that the string $c$ of the required polygon must pass through $O^{\prime}$, the intercept on a line through $O^{\prime}$ made by the strings $c$ and $f$ of this polygon will be $O^{\prime} S^{\prime}$, the corresponding intercepts of the first polygon being $R S$. Now, the ratio $\frac{O^{\prime} S^{\prime}}{R S}$ being constant for all corresponding intercepts of the two polygons (see Art. 20), the vertices of the required polygon may be determined as follows: Lay off $R^{\prime \prime} S^{\prime \prime}=R S$ (Fig. I9 A). From $S^{\prime \prime}$ describe an arc, radius $S^{\prime \prime} X=O^{\prime} S^{\prime}$ and draw the tangent $R^{\prime \prime} T$. Then to determine any intercept, for example $u v$, take the distance $u^{\prime} v^{\prime}$ in the dividers and step it off from $R^{\prime \prime}$ on $R^{\prime \prime} S^{\prime \prime}$. The perpendicular distance from the point thus located 
to $R^{\prime \prime} T$ is the desired intercept. The polygon drawn in full lines. was thus located.

In case the intercept $R S$ is shorter than $O^{\prime} S^{\prime}$, Fig. I9 A must be modified by making $R^{\prime \prime} S^{\prime \prime}=n \cdot R S$, where $n=2,3$, etc. In such case the proportion will be

$$
u v: O^{\prime} S^{\prime}:: n \cdot u^{\prime} v^{\prime}: n \cdot R S .
$$

The pole $P^{\prime}$ of the required polygon can be located on $P^{\prime} F$ (see Art. 22) by means of the proportion

$$
y^{\prime}: y:: R S: O^{\prime} S^{\prime} \text { (see Art. 20). }
$$

Problem. Draw a funicular curve (parabola) for a uniformly distributed load so as to pass through three given points (Fig. 20).
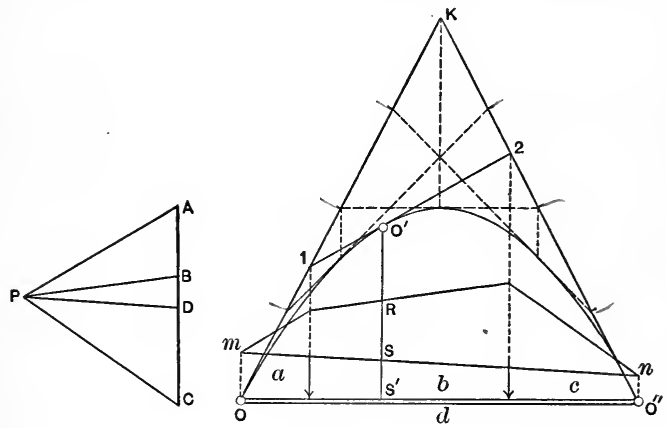

FIG. 20.

Let the load be distributed over the length $O O^{\prime \prime}$, the three points being $O, O^{\prime}$, and $O^{\prime \prime}$. Draw lines (vertical) parallel to the load through these three points. Substitute for the portions of the load lying on the two sides of the vertical through $O^{\prime}$, their resultants $A B$ and $B C$, and construct any funicular polygon $m n$ for them, $P$ being the pole. The corresponding funicular curve would be tangent to the strings $a, b$, and $c$ at $m, R$, and $n$ respect- 
ively (see Art. 13). A second funicular polygon whose string $b$ will pass through $O^{\prime}$ is now drawn, the vertices $I$ and 2 being located as explained for Fig. I9. The strings $O_{\mathrm{I}}, \mathrm{I}-2$, and ${ }_{2} \mathrm{O}^{\prime \prime}$ of this polygon will be tangent to the required funicular curve at $O, O^{\prime}$, and $O^{\prime \prime}$ respectively. This curve is constructed by the method shown in Fig. 9.

\section{Funicular Polygon through Two Points, One String having} a Given Direction. Problem. To draw a funicular polygon for a given system of forces, such that two designated strings shall pass through two given points and one string of the polygon shall have a given direction.

The method of Art. 22 can be used to determine the locus of the poles of all polygons passing through the two given points. The intersection of the ray corresponding to the string whose direction is given with this locus will be the pole of the required polygon.

If the string whose direction is given is also one of the two which are to pass through the given points, the following simpler solution can be made. Let $A B, B C$, and $C D$ (Fig. 2I) be the

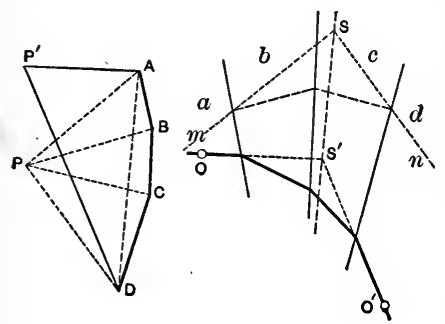

Fig. 21 . given forces, and $P$ the pole of any funicular polygon $m n$ for these forces. It is required to draw a second polygon such that the strings $a$ and $d$ will pass through $O$ and $O^{\prime}$ respecively, and the string $a$ be horizontal. The resultant of the forces included between the designated strings $a$ and $d$ is $A D$, its line of action passing through the 
intersection $S$ of the strings $a$ and $d$ of the first polygon. The corresponding strings of the second polygon must intersect on this line (Art. 8). These strings are, therefore, $O S^{\prime}$, drawn horizontally, and $S^{\prime} O^{\prime}$. The new pole $P^{\prime}$ will be at the intersection of the corresponding rays. The polygon can now be completed. 


\section{CHAPTER II.}

\section{R O OF-TRUSSES.}

\section{$\S$ I. Construction of Roofs, Loads, etc.}

26. Construction of Roofs. A roof includes the covering and the framework. The covering is tin, slate, tarred felt, corrugated iron, etc., laid over sheathing; or the sheathing may be omitted, as is commonly done when corrugated iron is used.

The roof covering is supported by a more or less elaborate framework, the principal members of which are often trusses or arches. A common arrangement of this framework, in case of
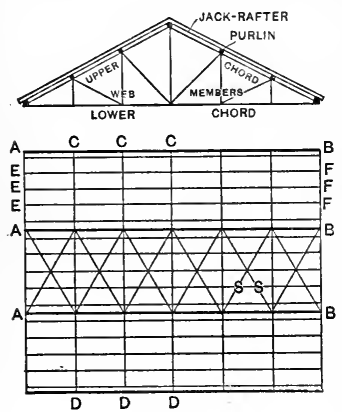

FIG. 22. shops, train-sheds, assembly halls, etc., is shown in plan and elevation in Fig. 22 The trusses, $A B$, are made up of an upper chord, a lower chord, and the web members. At right angles to the trusses and supported by them are a system of members, $C D$, called purlins. The purlins are usually wooden or iron beams, but in case of wide spacing between the trusses they may be trussed members. It is desirable to support the purlins at the joints of the upper chord, otherwise bending stresse; would occur in these members. Sometimes the covering is laid directly on the purlins, but more commonly jackrafters, $E F$, supported by the purlins, serve to further subdivide 
the area, the covering being laid on them. A system of swaybracing is employed, when necessary, to keep the trusses vertical and in line. Such a system is represented in Fig. 22 by the diagonal rods $S, S$, situated in the plane of the upper chord.

Roof-trusses are supported either by masonry walls or piers, or by wooden or iron columns. When iron trusses of considerable span rest on masonry, a more or less effectual provision for expansion consists in supporting one end on a planed bed-plate or on rollers.

(For a range of temperature of $120^{\circ} \mathrm{F}$. iron changes in length about I inch in $100 \mathrm{ft}$.) To provide for expansion lengthwise of an iron framed building, expansion joints are inserted in the longitudinal members at suitable intervals. ${ }^{1}$

27. Loads. The loads which have commonly to be provided for in designing a roof-truss are:

(I) Dead Load. This includes the weight of the covering, framework, and any other permanent loads supported by the truss, such as the weights of floors, ceilings, etc. This loading varies from about $8 \mathrm{lbs}$. to $35 \mathrm{lbs}$. or more per square foot of roof surface.

(2) Snow Load. This is estimated at ro lbs. to 25 lbs. per horizontal square foot in northern United States.

(3) Wind Load. This is estimated at 30 lbs. to $50 \mathrm{lbs}$. per square foot on a vertical surface, the resulting normal pressure

1 For detailed descriptions of roofs, see the following references:

"Revue Technique de l'Exposition de Chicago," Part I, Architecture (with Atlas). E. Bernard \& Co., Paris.

"Philadelphia and Reading Terminal Station, Philadelphia." Trans. Am. Soc. C. E., I 895 .

"The South Terminal Station, Boston, Mass." Trans. Am. Soc. C. E., Dec., 1899.

"St. Louis Coliseum." Jour. Assoc. Eng. Soc., May, I8o8.

"Dome of Government Building, World's Columbian Exposition." Trans. Am. Soc. C. E.. Jan., 1892. 
on an inclined surface being determined by some empirical formula. ${ }^{1}$

Provision should be made for any additional loads to be supported by the roof, e.g., live loads on floors, weights of shafting and travelling cranes, pull of belts, thrust of jib-cranes, etc.

The pressures at the various joints of a truss, due to the loading, are determined by the same methods as the supporting forces in case of a beam. 'The direct stresses (tension or compression in the members of a truss depend on these joint pressures or loads. When loads are applied to a truss at other points than the joints, the members of the truss thus loaded are subjected

${ }^{1}$ Read Lanza's "Applied Mechanics," $\$$ I30-I33, from which the following extract is taken:

"Duchemin's formula, which Professor W. C. Unwin recommends, is as follows, viz.:

$$
p=p_{1} \frac{2 \sin \theta}{\mathrm{I}+\sin ^{2} \theta}
$$

where $p=$ intensity of normal pressure on roof, $p_{1}=$ intensity of pressure on a plane normal to the direction of the wind.

"b) Hutton's formula,

$$
p=p_{1}(\sin \theta) \mathrm{r} .84 \cos \theta-\mathrm{I} .
$$

"Unwin claims that this and Duchemin's formula give nearly the same results for all angles of inclination greater than $15^{\circ}$.

"The following table gives the results obtained by the use of each, on the assumption that $p_{1}=40 . "$

\begin{tabular}{r|r|r||l|l|l}
\hline$\theta$ & Duchemin. & Hutton. & $\theta$ & Duchemin. & Hutton. \\
\cline { 2 - 5 } & 6.89 & 5.10 & $50^{\circ}$ & 38.64 & 38.10 \\
$5^{\circ}$ & 13.59 & 9.60 & $55^{\circ}$ & 39.21 & 39.40 \\
$15^{\circ}$ & 19.32 & 14.20 & $60^{\circ}$ & 39.74 & 40.00 \\
$20^{\circ}$ & 24.24 & 18.40 & $65^{\circ}$ & 39.82 & 40.00 \\
$25^{\circ}$ & 28.77 & 22.60 & $70^{\circ}$ & 39.91 & 40.00 \\
$30^{\circ}$ & 32.00 & 26.50 & $75^{\circ}$ & 39.96 & 40.00 \\
$35^{\circ}$ & 34.52 & 30.10 & $80^{\circ}$ & 40.00 & 40.00 \\
$40^{\circ}$ & 36.40 & 33.30 & $85^{\circ}$ & 40.00 & 40.00 \\
$45^{\circ}$ & 37.73 & 36.00 & $90^{\circ}$ & 40.00 & 40.00 \\
\hline
\end{tabular}


to bending stresses in addition to the direct stresses. This case is discussed in $\$ 6$.

The direct stresses in the members of a roof-truss can usually be determined most readily by graphical methods. In order to use such methods to the best advantage, however, it is necessary to freely employ algebraic methods in connection with them, as indicated in the following pages.

\section{§. Determination of Reactions of Supports.}

28. Cases. Let the loads acting on the truss (Fig. 23) be resolved into horizontal $(H)$ and vertical $(V)$ components, $\Sigma H$ and $\Sigma V$ representing the algebraic sums of the horizontal and vertical component loads respectively, and $\Sigma M$ the algebraic sum of the moments of the loads. Let $H_{1}, V_{1}$, and $H_{2}, V_{2}$ be the components of the left and

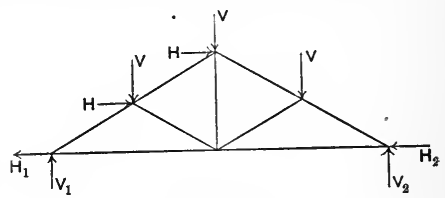

Fig. 23. right reactions respectively. Then we have

$$
\begin{aligned}
& H_{1}+H_{2}=\Sigma H \text {, ・ ・ ・ } \\
& V_{1}+V_{2}=\Sigma V ; \cdot \bullet \cdot \bullet \cdot \cdot
\end{aligned}
$$

and, taking moments about the right support,

$$
V_{1} l=\Sigma M, \cdot \cdot \cdot \cdot \cdot \cdot \cdot \cdot
$$

in which $l=$ span.

These three equations involve four unknown quantities. From equations (2) and (3) $V_{1}$ and $V_{2}$ can be determined; their values are thus independent of the manner of supporting the ends of the truss. The values of $H_{1}$ and $H_{2}$, however, vary with the manner of supporting the truss. The following cases occur: 
CASE I. Truss fixed in position at both supports by anchorbolts or otherwise. When the roof is comparatively flat, so that the resultant load, including wind pressure, is nearly vertical, the reactions can be assumed parallel to each other, and consequently parallel to the resultant load. In the case of steep roofs, or in general where the resultant load makes a large angle with the vertical, this assumption may lead to absurd results.

The assumption which probably approaches the truth as closely as can be expected for so indeterminate a case is that the horizontal reactions of the two supports are equal. If the truss is assumed to be rigid, the supports equally elastic, and no other forces besides the horizontal pressure on the truss in action to cause the supports to yield, this assumption would be correct; for, since the supports yield equally, the horizontal forces causing such yielding must be equal.

When the truss is not anchored to its supports, the conditions are evidently similar to Case I, so long as the weight and friction are sufficient to prevent motion.

CASE II. One end of truss supported on rollers, planed bedplate, or similar device to provide for expansion. If friction be neglected, the reaction at the free end is evidently vertical. Friction at the free end may be dealt with as follows: First determine the supporting forces on the assumption that both ends are fixed. If the resulting reaction at the free end makes an angle with the vertical less than the angle of repose, these reactions are the true ones. If, however, the reaction at the free end, thus determined, makes a greater angle with the vertical than the angle of repose, both reactions must be determined anew on the assumption that the reaction at the free end makes an angle with the vertical equal to the angle of repose.

CASE III. Truss supported on columns. This case is discussed in $\S 6$. 
29. Examples. The methods of determining the supporting forces are included under Arts. 5, 15, and 16. A few examples are given to indicate suitable solutions.

Example I. The truss (Fig. 24) is subjected to wind pressure from the left, the load being uniformly distributed over the rafter. Find reactions of supports: (I) When both ends are fixed (Fig. $24 \mathrm{~A}$ ); (2) When the right end is supported on rollers (Fig. $24 \mathrm{~B}$ ); (3) When the left end is supported on rollers (Fig. 24 C).

The resultant load acts at the middle of the rafter. In Case (I) the reactions are assumed to be parallel. They are found, by the method of Art. 15, also by dividing the resultant load, $A D$, into parts inversely proportional to the segments into which the line of action of the resultant load divides the line joining the supports.

The method of Art. I6 is used to solve Cases (2) and (3), the reaction at the free end being assumed vertical. The line of action of the re-
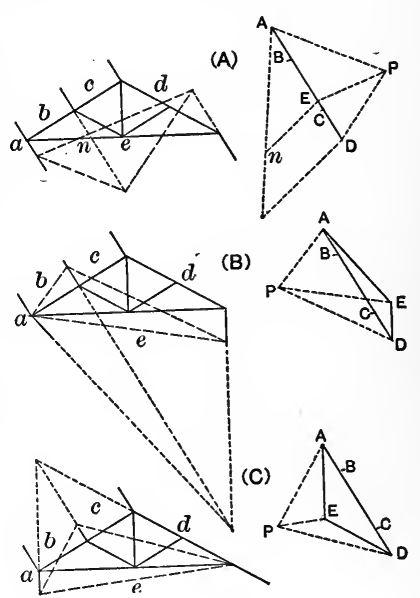

FIG. 24. action at the fixed end is also determined from the condition that three non-parallel forces in equilibrium must intersect at the same point, after which the magnitudes of the reactions can be determined by the triangle of forces.

The vertical components of the reactions are the same for all three cases (see Art. 28); hence the results for any one of these 
cases might have been used in solving the other two cases (see Example 2).

Example 2. In the truss (Fig. 25) $A B$ and $B C$ are the resultant wind loads on the vertical and inclined surfaces respectively, and $C D$ is the resultant dead load. It is required to determine the supporting forces, making use in turn of each of the following assumptions:

(I) Left end supported on rollers, reaction vertical; (2) left end supported on smooth bed-plate, coefficient of friction $=\frac{1}{3} ;(3)$ both ends fixed, reactions parallel; (4) both ends fixed, horizontal
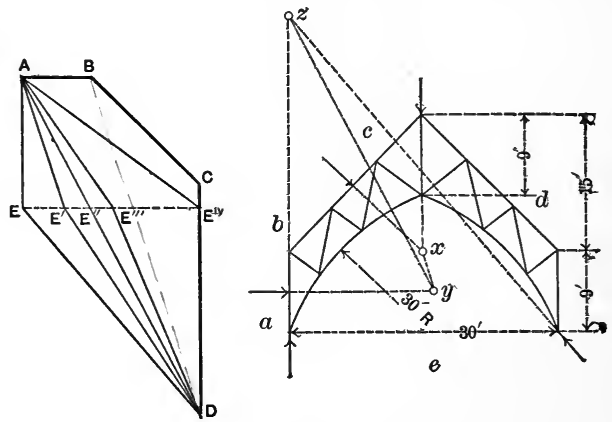

FIG. 25 .

reactions equal; (5) right end supported on rollers, reaction vertical.

Solution. The line of action of the resultant of $B C$ and $C D$ is $x y$, drawn through $x$ parallel to $B D$. This resultant combined with $A B$ gives $z y$ for the line of action of the resultant load $A D$. The supporting forces under assumption I, determined by the second method used in Example I for Cases 2 and 3, are $D E$ and $E A$. 
Now, since the vertical component reactions are independent of any assumption, the point corresponding to $E$ must lie on a horizontal line through $E$ in all cases. Hence the remaining cases can be solved as follows:

Assumption 2. Lay off $E E^{\prime}=\frac{1}{3} E A$, then $D E^{\prime}$ and $E^{\prime} A$ are the supporting forces.

Assumption 3. $D E^{\prime \prime}$ and $E^{\prime \prime} A$ are the supporting forces.

Assumption 4. Bisect $E E^{\text {iv }}$ at $E^{\prime \prime \prime}$, then $D E^{\prime \prime \prime}$ and $E^{\prime \prime \prime} A$ are the supporting forces.

Assumption 5. $D E^{\text {iv }}$ and $E^{\text {vv }} A$ are the supporting forces.

It should be noted that if the supporting forces had been determined for the dead and wind loads separately and the results combined, the resultant reactions thus found would differ from those in Example 2 when assumptions 2 and 3 are employed, but not in the other cases.

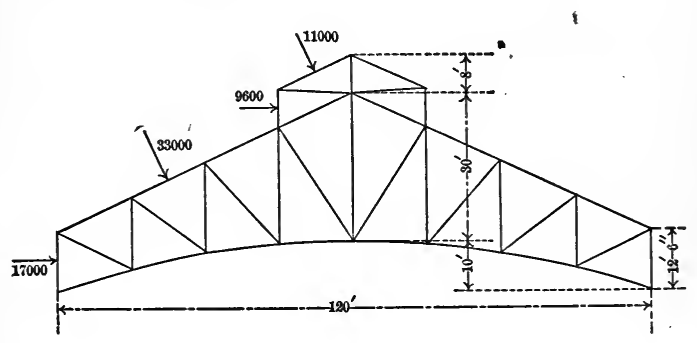

FIG. 26.

Problem. The truss (Fig. 26) is subjected to wind pressure on the left side as shown, in addition to a uniformly distributed vertical load of $75,000 \mathrm{lbs}$. Determine the supporting forces under each of the assumptions of Example 2.

30. Algebraic Solution. The student should be able to determine reactions algebraically as well as graphically. The 
general method is indicated in Art. 28. Equations (2) and (3) (Art. 28) serve to determine $V_{1}$ and $V_{2}$. In order to determine $H_{1}$ and $H_{2}$, we must have, in addition to Equation (I), a second equation based on the assumption employed. Taking the five assumptions of Example 2 in order, this second equation is as follows:

Assumption I. $H_{1}=0$.

$\begin{array}{lll}\text { “ } & \text { 2. } H_{1}=\frac{1}{3} V_{1} \cdot \\ & \text { 3. } & \frac{H_{1}}{V_{1}}=\frac{H_{2}}{V_{2}}=\frac{\Sigma H}{\Sigma V} . \\ \text { “ } & \text { 4. } & H_{1}=H_{2} . \\ \text { " } & \text { 5. } & H_{2}=0 .\end{array}$

Problex. Solve the problem (Fig. 26) algebraically.

\section{§3. Determination of Stresses.}

31. General Methods. A truss is designed to support loads applied at the joints by vir.ue of the resistance to extension and compression of its various members.

There are two general methods for determining the tension and compression stresses in the members of a truss: (I) method of sections; (2) method of joints.

32. Method of Sections. Let the imaginary line $x y$ (Fig. 27) divide the truss into two parts, this line intersecting the three

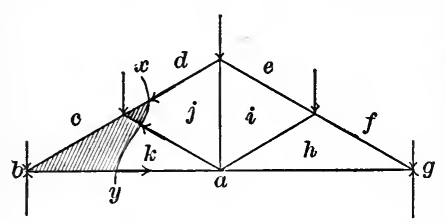

Frg. 27. members $d j, j k, k a$. The portion of the truss to the left of $x y$ is a body in equilibrium under the action of certain forces. Consider the member $d j$. If it is in tension, the portion to the right of $x y$ must be exerting force upon the portion to the 
left of $x y$, toward the right. If $d j$ is in compression, its righthand portion must, on the other hand, be exerting force upon its left-hand portion, towards the left. In either case, the magnitude of this force is equal to that of the stress in the member, and the line of action of the force has the direction of the length of the member. This force is external with reference to the portion of the truss to the left of $x y$. It will be represented by the letters $d j$. Similar explanations hold for $j k$ and $k a$. The forces, therefore, which hold the left portion of the truss in equilibrium are the known forces $a b, b c, c d$, and the forces $d j, j k$, and $k a$, exerted by the right-hand portions of these three members upon their lefthand portions. The lines of action of these last three forces are known, their magnitudes and directions being unknown. These six forces constitute a system of forces in equilibrium, lying in the same plane, but not acting at the same point. One or more of the conditions of equilibrium of Art. 5 can therefore be used to determine the unknown forces, as was indicated in Art. 6. The third solution of Art. 6 (Example 2) is generally most useful in dealing with roof-trusses.

The following points should be noted:

(I) Only three forces, unknown in magnitude, can be determined, so that if the section $x y$ cuts more than three members which are in action under the given loads, a solution cannot be made.

(2) The stress in a member is equal to the magnitude of the force which it exerts, and the nature of the stress, tension, or compression can be determined from the direction of the force (tension, if directed away from left portion; otherwise compression).

33. Method of Joints. The external forces acting upon the joint $b$ (Fig. 27) are: (I) the supporting force $a b$; (2) the load 
$b c$; (3) the forces exerted by the members $c k$ and $k a$ upon the joint. If either member as $c k$, is in tension, the force which it exerts on the joint $b$ is evidently directed away from the joint; if in compression, towards it. As the joint is in equilibrium, these four forces must balance, and by applying either the algebraic method of resolution of forces or the geometric method of polygon of forces, the unknown forces can be determined.

In using the method of joints, the following points should be noted:

(I) The forces dealt with are those acting on the joint.

(2) In dealing with any one joint only two unknown forces can be determined.

(3) The nature of the stress in a member can be determined from the direction of the force which the member exerts on the joint: tension, if the force acts away from the joint; compression, if the force acts towards the joint.

(4) When the stress in a member is determined, the force which it exerts upon the joint at each end is known, these forces being equal and opposite.

34. Determination of Stresses in Roof-trusses. Of the methods which have been explained, the one best adapted to rooftrusses is the method of joints, solving by the polygon of forces. In applying this method, the external forces being known, we begin by constructing the polygon of forces at any joint of the truss where only two stresses are unknown. Having thus determined these two, we repeat the construction for another joint where only two stresses remain unknown, and continue in this manner until the stresses in all the members have been determined.

35. Example. Bow's Notation. The truss (Fig. 28) is sub. jected to a uniformly distributed load, $W$. Each intermediate 
joint of the upper chord supports $\frac{1}{4} W$, and each end joint $\frac{1}{8} W$. Each supporting force is $\frac{1}{2} W$. The external forces are lettered as in the preceding chapter, $a b$ representing the left reaction; $b c, c d$, etc., the successive loads. Letters are also placed in the spaces into which the surface is divided by the web members. Each member of the truss is represented by the letters in the adjacent spaces; e.g., the two halves of the lower chord are $k a$ and $h a$; the vertical member is $j i$,

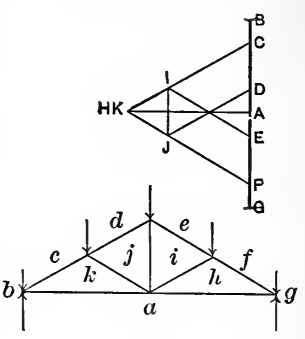

FIG. 28. etc. The directions of the forces are indicated in the force (or stress) diagram by the order of the letters. For this purpose the letters are to be read in right-handed order around the truss or any joint of it; e.g., the left reaction is $a b$, left-hand load $b c$, etc.; the force exerted by the member $c k$ upon the left-hand joint is $c k$, and upon the joint $c d j k$ is $k c$.

To illustrate the manner of using this notation, the stresses in the various members of the truss will be determined. First construct the polygon of external forces. Lay off the loads on a vertical line in right-handed order. The first load, beginning at the left, is $b c$; it acts downwards, and to indicate this the force is lettered so that $B C$ reads downwards. Continuing in this manner, when the polygon is completed, if we read the letters surrounding the truss in right-handed order, bcdefgab, these letters in the stress diagram, read in the same order, will represent the polygon of external forces, the order of succession of the letters indicating the direction in which the forces act.

Next the polygon of forces for the left-hand joint of the truss is constructed. The known forces $a b$ and $b c$ are already included in the stress diagram. The two unknown forces are $c k$ and $k a$. 
One letter of each $(C$ and $A$ ) is already in the stress diagram. These letters indicate the points from which to draw the lines representing the unknown forces. Thus the polygon is completed by drawing from $C$ and $A$ lines parallel to $c k$ and $k a$ respectively, their point of intersection being lettered $K$. Proceeding to the next joint, $k c$ and $c d$ are the known forces. From the points $D$ and $K$ lines are drawn parallel to $d j$ and $j k$ respectively, intersecting at $J$, thus completing that polygon. By this mode of construction, the polygons of forces for the various joints of the truss are grouped together in a single diagram called the stress diagram. When the last joint is reached, all the stresses but one will be known. The letters representing this one will already be in the stress diagram, and the line joining them will be parallel to the corresponding member of the truss if the construction is accurate.

The following points should be noted in using Bow's notation:

(I) The stress in any member of the truss is represented by the same letters as the member itself.

(2) The polygon of forces for any joint is lettered with the letters surrounding that joint, the direction of the forces being indicated by the succession of letters obtained by reading the letters surrounding the joint in right-handed order.

(3) To determine whether any member is in tension or compression, read the letters representing that member in righthanded order about the joint at either end of the member. The same order of succession of these letters in the stress diagram indicates the direction of the force which the member exerts on the joint used. If this direction is towards the joint, the stress is compression; if away from it, tension. As an illustration, let the nature of the stress in $c k$ be determined. Using the left joint, the letters read $c-k$. In the stress diagram this order of succession of these letters is towards the left. Referring again to the 
truss, the direction, towards the left, is seen to be towards the joint used as a centre, this indicating that the member $c k$ is exerting force on this joint towards it. Hence $c k$ is in compression.

(4) In constructing the polygons of forces it is to be noted that all the sides of any polygon but the two corresponding to the two unknown forces at the joint will be already represented in the stress diagram, and the points from which to draw these two sides are indicated by the lettering, as previously explained. The point of intersection of these two sides is marked with the letter common to the corresponding members of the truss.

(5) Referring to Fig. 27, any portion of the truss (e g., the shaded portion) is a body in equilibrium. The external forces: acting on it must then form a polygon which would be lettered with the letters surrounding it (e.g., $a b c d j k a$ ). It should be: observed that all such polygons are included in the stress diagram: (see Fig. 28). Hence the same stress diagram would be obtained whether the method of joints or method of sections were employed in constructing the various force polygons.

In using the method illustrated, the truss is drawn accurately to scale, the loads are also plotted accurately to scale, and the. stress diagram is constructed with extreme care. The magni-tudes of the stresses are gotten by scaling off the proper lines of the: stress diagram, and the nature of the stress is derived from the lettering, as previously explained. The most serious cause of inaccuracy, especially in case of trusses having a large number of members, is that the construction of each polygon, in turn, is based upon preceding ones, so that errors accumulate. To guard against this, the method of sections should be employed as indicated in Art. 37 .

The student is recommended to study trusses with the view of understanding the purpose and action of their various members independently of the stress diagrams. In Fig. 28 , for example, the 
load $c d$ tends to deflect the rafter, this deflection being prevented by the brace $k j$, which would therefore be in compression. $k j$, being in compression, exerts a downward thrust on the lower chord at $a$. The lower chord is prevented from deflecting under this thrust by $j i ; j i$ is therefore in tension. A study of trusses in such a manner will assist the student to a better understanding of their design.

36. Determination of Maximum and Minimum Stresses. In order to proportion the various parts of a truss, it is necessary to know the extreme range of the stresses to which each member will be subjected under the various combinations of loads liable to occur. The loads to take into account will vary with the circumstances. In case of the arches supporting the train-shed roof of the Philadelphia and Reading Terminal Station, Philadelphia, the stresses were determined for: (I) dead load; (2) dead load and snow on one side; (3) dead load and snow on both sides; (4) dead and wind loads; (5) dead, snow, and wind loads. ${ }^{1}$ On the other hand, the train-shed trusses of the South Terminal Station, Boston, were designed to support a uniformly distributed vertical load only. ${ }^{2}$ The most usual combinations are: (I) dead load; (2) dead and snow loads; (3) dead and wind loads.

There are two methods of procedure in this connection: (I) Construct a separate stress diagram for each kind of loading, and combine the stresses thus determined; (2) Construct a diagram for each combination of loads. The student should be familiar with both methods; the latter, however, appears to be generally preferable for the following reasons: (I) Under certain assumptions the first method does not give correct results; e.g., under the assumption of parallel reactions, if the reactions for dead

${ }^{1}$ See Trans. Am. Soc. C. E., Aug., 1895 .

${ }^{2}$ Ibid., Dec., 1899. 
and wind loads, considered separately, are assumed to be parallel, the resultant reactions found by combining these will in general not be parallel. Again, in dealing with friction at the supports, the two methods will give different reactions, and consequently different stresses. (2) In dealing with counterbracing (\$4), the second method is the more simple of the two; (3) When the second method is employed, the maximum and minimum stresses can be scaled directly from the diagrams.

\section{Example. Wooden Truss fixed at Both Ends. Figure} I, Plate I, represents one of a series of parallel trusses, spaced I6 ft. between centres, supporting a roof. The vertical iron tie-rods divide it into eight panels of equal width. $S p a n=80 \mathrm{ft}$. Rise of upper chord $=\mathrm{I} 6 \mathrm{ft}$. The roof is to be covered with tin laid on sheathing I in. thick. The sheathing is supported by rafters 2 in. by 7 in. section, spaced two feet between centres. The rafters are supported by purlins 8 in. by $\mathrm{I} 2 \mathrm{in}$. section, these being supported at the joints of the upper chord.

Each rafter supports an area of the roof surface 2 feet wide by $10.8 \mathrm{ft}$. long, and is proportioned as a beam to support the maximum load, including its own weight, which is liable to come upon this area. Each intermediate purlin supports an area Io. $8 \mathrm{ft}$. wide by $16 \mathrm{ft}$. long, and is also proportioned as a beam for the maximum load to which it is liable to be subjected. The student should verify these dimensions of rafters and purlins, using a working stress of $1000 \mathrm{lbs}$. per sq. in.

\section{Calculation of Loads.}

Wt. of tin and sheathing per sq. ft. of roof surface $=3 \frac{1}{2} \mathrm{lbs}$.

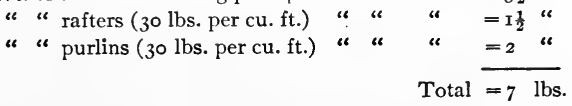


Estimated weight of truss $=5760$ lbs. This weight is assumed to be supported at the joints of the upper chord.

(The weight of the truss must be estimated from the actual weights of trusses of similar design and loading already built. Merriman gives the formula for wooden trusses, $W=\frac{1}{2} a l\left(\mathrm{I}+\frac{1}{10} l\right)$, and for iron trusses $W=\frac{3}{4} a l\left(\mathrm{I}+\frac{1}{10} l\right)$. These formulas are derived from a table of weights of trusses given by Ricker. Johnson gives, for iron trusses, the formula $W={ }_{2}^{1} \frac{1}{4} a l^{2}$. In each case $W=$ weight of truss in pounds, $a=$ distance between trusses in feet, $l=$ span in feet. These formulas can, at the best, be only rough approximations.)

The snow load is assumed to be 20 lbs. per sq. $\mathrm{ft}$. of horizontal projection of roof surface, and the wind pressure $40 \mathrm{lbs}$. per. sq. ft. on a vertical surface.

Normal component of wind pressure (Hutton's formula) $=$ 20 lbs. per sq. ft. of roof surface.

LoAds at INTERmediate JOINTS OF UPPER ChORD.

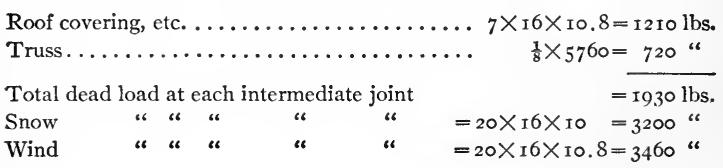

Each end joint supports one-half the load carried by an intermediate joint.

The loadings considered in this example are: (I) dead load; (2) dead and snow loads; (3) dead and wind loads. The student should trace out the construction of the diagrams, noting the steps taken to secure accuracy and to check the work.

Dead-load Diagram (Fig. I B). The reaction of each support is 7720 lbs. The total load is plotted and subdivided to 
obtain the joint loads. Since the truss is symmetrical and symmetrically loaded, the stresses in corresponding members on the two sides will be equal; the diagram is therefore constructed for one side only. In order to avoid a long succession of joints and to check the work, the stresses in $e r, r q$, and $q k$ are determined at the outset by the method of sections (Art. 32) as follows :

Calculate the stress in one of these members, as $q k$, by moments. Taking the moment axis at the intersection of the other two members, we have

$7720 \cdot 30-965 \cdot 30-1930(20+10)=12 Q K . \quad \therefore Q K=12,062$ lbs.

The moment of the known forces being right-handed, the moment of $Q K$, to balance, must be left-handed, hence $Q K$ is in tension since the force acts away from the portion of the truss under consideration. Next plot this computed stress in the diagram, laying off from $K$ the length $Q K$ in such direction that it will represent tension. The stresses in $e r$ and $r q$ are next determined by completing the polygon $Q K A B C D E R Q$. The remaining stresses to the left of the section are determined by the method of joints as follows:

At joint I determine $B L$ and $L K$; at joint 2, $L M$ and $M K$; at joint $3, C N$ and $N M$; at joint $4, D P$ and $P Q$; at joint $5, P O$ and $O K$. If, now, the closing line $N O$ is parallel to the member no, the work is checked. By this order of procedure a long succession of force polygons dependent on each other has been avoided, thus preventing inaccuracies from accumulating, and the work has been completely checked. Trusses should be divided in this manner by a sufficient number of sections so that each division will contain not more than ten or twelve joints. It should be noted that the mere closing of the force polygons, without the introduction into the stress diagram of stresses computed independently of the diagrams, does not completely check 
the work, since errors made in constructing the truss diagram and plotting th: loads would not interfere with the diagrams closing.

Dead and Snow Loads. Since, in this example, the snow load is distributed in the same manner as the dead load, the stresses can be found by multiplying the dead-load sitresses by $\frac{1930+3200}{1930}$.

Dead and Wind Loads (Fig. I A). The wind is taken to come from the right. Attention is directed to the following points:

(I) The resultant dead and wind loads are first plotted, as shown by the dotted lines, the joint loads being found by suitable subdivision.

(2) The resultant load intersects the lower chord at $z$, the reactions $J K$ and $K A$ (assumed to be parallel) being found by dividing $A J$ into parts inversely proportional to the segments of the lower chord.

(3) The stresses are determined in a manner similar to that explained for the dead load. In calculating the stress in $q k$ it is convenient to know the $H$ and $V$ components of the external forces.

$H$ component of wind load $=\frac{\mathrm{I} 6}{43.08} \cdot \mathrm{I} 3840=5^{\mathrm{I} 40 \mathrm{lbs} .}$

$V$ component of wind load $=\frac{40}{43.08} \cdot \mathrm{I} 3840=\mathrm{I} 2850 \mathrm{lbs}$.

Taking moments about the right-hand support,

$$
80 V_{1}=\mathrm{I} 5440 \cdot 40+\mathrm{I} 2850 \cdot 20+5 \mathrm{I} 40 \cdot 8 . \quad \therefore V_{1}=\mathrm{II} 446 \mathrm{lbs} .
$$$$
H_{1}=\frac{5^{\mathrm{I} 40}}{\mathrm{I} 5440+\mathrm{I} 2850} \cdot \mathrm{II} 446=2080 \mathrm{lbs} .
$$ 


$$
\begin{aligned}
Q K & =\frac{\mathrm{II} 446 \cdot 30-2080 \cdot \mathrm{I} 2-965 \cdot 30-\mathrm{I} 930(20+\mathrm{IO})}{\mathrm{I} 2} \\
& =\mathrm{I} 9300 \mathrm{lbs} .(\text { tension }) .
\end{aligned}
$$

Maximum and Minimum Stresses. The maximum and minimum stresses for the different members of the truss were determined as follows: Tabulate the stresses in all the members, for each of the three combinations of loads. Note that the extreme range of stresses will be the same, in case of corresponding members on the two sides of the truss. Thus, considering the members $o p$ and $u v$, we find the stresses (in pounds) recorded in the following table:

\begin{tabular}{|c|c|c|c|}
\hline & Dead. & $\begin{array}{c}\text { Dead and } \\
\text { Snow. }\end{array}$ & $\begin{array}{c}\text { Dead and } \\
\text { Wind. }\end{array}$ \\
\hline \multirow{2}{*}{$o p$} & $\begin{array}{c}+3100 \\
+3100\end{array}$ & $\begin{array}{l}+8200 \\
+8200\end{array}$ & $\begin{array}{l}+3100 \\
+9100\end{array}$ \\
\hline
\end{tabular}

Of these stresses, the extremes are 9roo lbs. and 3100 lbs. (see Fig. I C). Each of these two members must then be designed to support a compression stress of 9100 lbs., the minimum stress, 3100 lbs., being also used if the effect of repetition of stress is considered in such design.

The maximum and minimum stresses in all the members are recorded in Fig. I C. These should be verified by the student.

If the truss is unsymmetrical, supported differently at the two ends, etc., the above procedure must evidently be modified.

38. General Remarks. I. When the loading is complicated, special precautions should be taken to plot the joint loads in such a manner as to avoid any accumulation of errors. This may be accomplished as indicated in Art. 37, otherwise as follows (see 
Fig. 29): Having resolved the loads into $H$ and $V$ components, plot

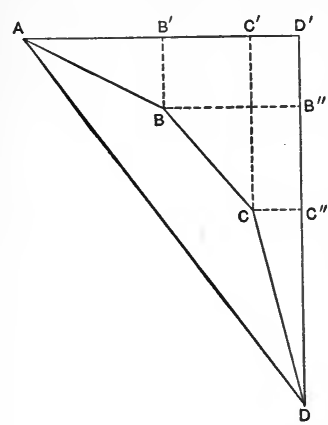

FIG. 29. $A D^{\prime}=\Sigma H$ and $D^{\prime} D=\Sigma V$, then $A D$ is the resultant load. To plot the joint loads, lay off on $A D^{\prime}$ the $H$ component joint loads $A B^{\prime}, B^{\prime} C^{\prime}$, etc., in order, and on $D^{\prime} D$ the $V$ component joint loads $D^{\prime} B^{\prime \prime}, B^{\prime \prime} C^{\prime \prime}$, etc., in the same order. Combining these we have $A B, B C$, etc., for the resultant joint loads.

2. The precaution, illustrated in Art. 37 , of computing the stress in one or more of the members by the method of sections, and plotting the results in the stress diagram, should always be employed in large or important work.

3. In constructing stress diagrams it is sometimes desirable to obtain the directions of the members more accurately than can be done by using the truss diagram unless the latter is constructed on an inconveniently large scale. For this purpose coordinate axes can be used, the slopes of the various members being plotted on them as indicated for st and $u v$ (Fig. I D). A good general rule to follow is to avoid obtaining the direction of a line of the stress diagram from a shorter line of the truss.

4. Nothing is gained by constructing the stress diagram on a very large scale. The lines of such diagrams need not be in error more than two or three hundredths of an inch. With a scale of $10,000 \mathrm{lbs}$. to the inch this would be an error of 200 to $300 \mathrm{lbs}$. in the stress. Such an error is of no importance in work requiring so small a scale.

5. The diagrams should be constructed with such care and - in such a manner as to leave no question as to their accuracy at any stage of the construction. The wooden edges of the ordinary 
drawing-board and $\mathrm{T}$ square are unreliable. A steel straightedge with lead weights to hold it in position is preferable. The edges of the triangles must be straight and the $90^{\circ}$ angle true. The usual hard-rubber and celluloid triangles are not sufficiently accurate in these respects and should be tested before using. The best quality of dividers and compasses, a metal scale gradated to hundredths of an inch, and a hard pencil kept sharpened to a fine chisel edge, complete the list of necessary instruments. All intersections to be preserved are located by a fine prick-point enclosed in a circle.

39. Problems. I. The truss, Fig. 30 , fixed at both ends, is one of a system of parallel trusses, spaced to ft. apart, support-

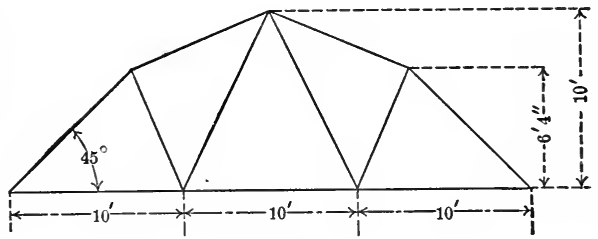

Fig. 30 .

ing a roof. The dead load is 20 lbs. per sq. ft. of roof surface. The wind pressure is assumed to be $40 \mathrm{lbs}$. per sq. $\mathrm{ft}$. on a vertical surface. Determine the stresses in all the members due to the combined dead and wind loads.

Directions. (I) Assume the $H$ component reactions to be equal. (2) Determine the normal wind loads on the two inclined roof surfaces by Hutton's or Duchemin's formula (see table, p. 32 ). (3) Plot these two wind loads together with the resultant dead load and determine the reactions of supports by drawing a funicular polygon for these (three) resultant loads (see Arts. ${ }_{5}$ and 
29). (4) Plot the joint loads in order (see Art. 37) and draw the stress diagram, checking on the middle portion of the lower chord.

Problem 2. The truss, Fig. $3^{\mathrm{I}}$, is loaded on the upper chord . with a uniformly distributed dead load of 75,000 lbs. In addi-

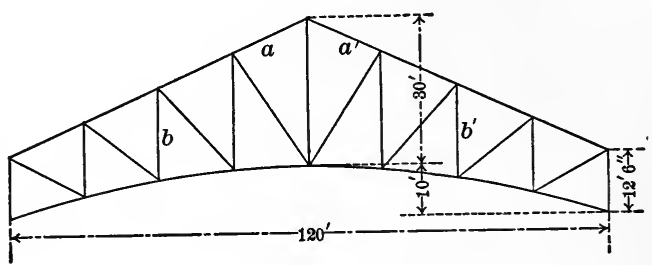

FIG. 3I.

tion, each intermediate joint of the lower "chord is loaded with $2000 \mathrm{lbs}$. The lower chord is a circular arc. The vertical web members are equidistant. Determine the stresses in all the members.

Directions. (I) In plotting the truss, locate the joints of the lower chord by ordinates. (2) Plot the external forces in right-handed order as usual, namely, loads on upper chordright-hand reaction-loads on lower chord-left-hand reaction. (3) Calculate stress in member $a$ (or $a^{\prime}$ ) by moments, plotting it in the stress diagram. (4) Construct the stress diagram for either half-truss, checking on $b$ (or $b^{\prime}$ ).

Problem 3. Determine the maximum and minimum stresses in the truss, Fig. 3I, providing for a wind pressure of $40 \mathrm{lbs}$. per sq. ft. on a vertical surface as well as for the dead loads of Prob. 2. Assume that one end of the truss rests on rollers, the reaction at that end being vertical. (Note. Three combinations of loads must be dealt with, namely: dead load alone; 
dead load with wind on roller side; dead load with wind on fixed side.)

40. Cantilever Truss. Fig. 32, which represents one of the train-shed trusses of the South Terminal Station, Boston, con-

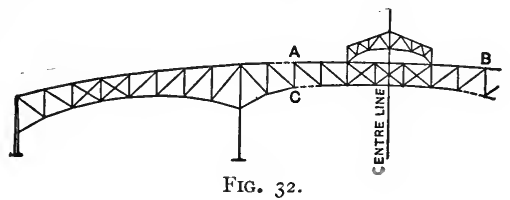

sists of a central truss, $A B$, supported at $A$ and $B$ on the ends of the cantilevers. $A C$, acting as a link, provides for expansion. The dotted chord members are furnished with sliding joints and thus do not resist tension or compression. The stresses can be determined in the usual way, first finding the reactions at $A$ and $B$ due to the loads on the central truss. ${ }^{1}$

4I. Trusses having only Two Forces to determine at Each Joint. In the preceding examples only two unknown stresses were encountered at each joint, so that the polygons of forces for the successive joints of the truss could be constructed at once. Other cases of the same nature are given in Fig. 33 A, B, C, D, E, F, G, and H. The student should verify this statement, indicating the order of succession of joints to use in constructing the stress diagrams.

42. Fink, or French, Roof-truss. This is a type of iron truss in common use for shops and similar buildings. Fig. $33 \mathrm{~L}$ represents its most usual form. In applying the method of joints

${ }^{1}$ See Trans. Am. Soc. C. E., Dec., 1899. 
54

GRAPHIC STATICS.
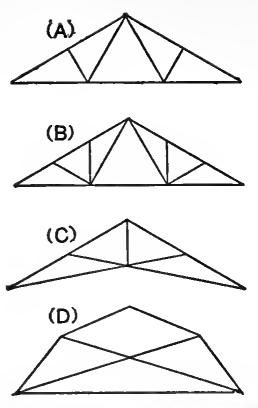
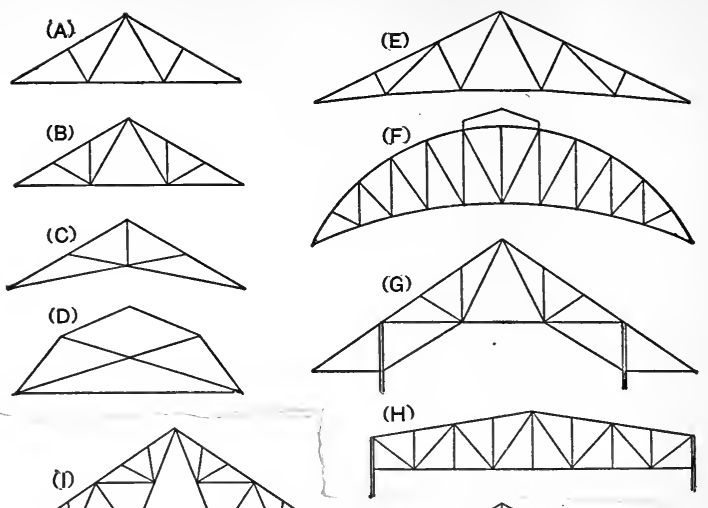

(L)
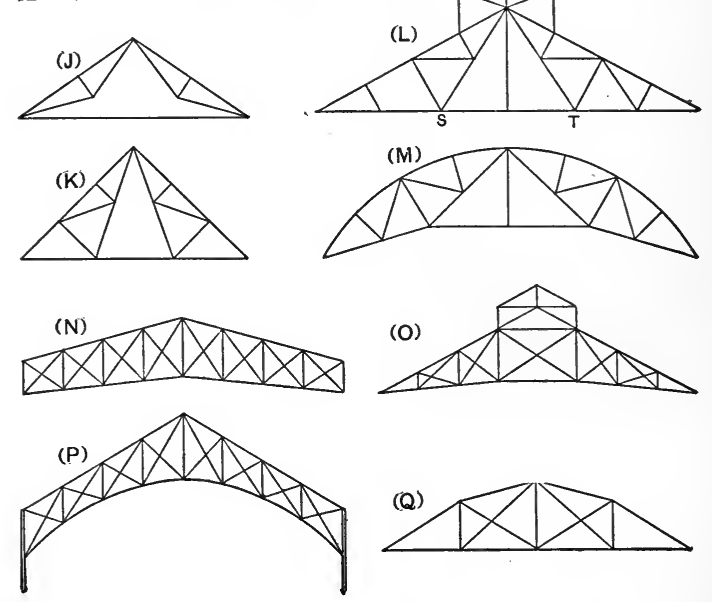

FIG. 33 
to this truss a difficulty is encountered to which attention is directed. Beginning at the left-hand support, the force polygons can be constructed in the usual manner until the joint $S$ is reached, where three unknown forces are encountered. There are various graphical devices for overcoming the difficulty; the most satisfactory solution, however, is to calculate the stress in $S T$ by moments (method of sections) and insert this calculated stress in the diagram. The stresses in the two remaining members at the joint $S$ can then be found by completing the force polygon. If the method of procedure of Art. 37 is followed no difficulty arises. Other trusses of the same nature are shown in Fig. $33 \mathrm{I}, \mathrm{J}, \mathrm{K}$, and $\mathrm{M}$.

\section{§4. Counterbracing and Double Systems of Bracing.}

Trhere Mar

43. Definitions. It will be noticed that the members of a properly designed truss form in general a system of triangles. The triangle is the elementary truss. Under the action of forces lying in its plane and acting at its vertices, it cannot be distorted without changing the lengths of one or more of its sides, and such changes are opposed by the resistance to extension and compression of the sides. A polygonal frame of more than three sides, assumed free to turn at the joints, can be distorted without altering the lengths of any of the sides.

The quadrilateral frame (Fig. 34), acted on by the force $F$, would be distorted as shown, the distance $F$ $A B$ becoming shorter and $C D$ longer, change in length of the diagonals necessarily accompanying the distortion of the frame. A diagonal member capable of resisting both extension and compression would, then, prevent distor-

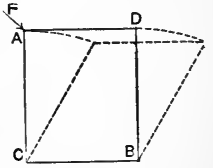

FIg. 34. tion. A member joining $C$ and $D$, capable of resisting tension 
alone, would prevent distortion in the direction shown in Fig. 34, but not in the opposite direction. Two tension diagonals, however, would evidently make the frame stable. Thus a quadrilateral frame may be made capable of resisting any forces acting at the angles, tending to distort it, by the introduction of a single diagonal member capable of resisting both tension and compression, or of two diagonals, both capable of resisting tension alone or compression alone. In the latter case it is evident that only one of the diagonals would be stressed at a time. Considering the quadrilateral of Fig. 34 to represent one panel of a truss, the diagonal which is stressed under the action of the dead load may be called the main brace, the other the counterbrace or counter. The counter may be stressed under the action of the wind, snow on one side of the roof, or other non-symmetrical temporary load.

44. Notation. The system of notation of Fig. 35 is convenient to use for trusses with counterbracing. One diagonal of each panel is drawn dotted. The diagonals drawn in full lines are designated by the letters $g h$ and $i j$, the same letters accented being used for the dotted diagonals. To illustrate, if the diagonals under stress were $g^{\prime} h^{\prime}$ and $i$, the verticals would be represented by $f h^{\prime}, g^{\prime} i$, and $j k$; the members of the upper chord would be $b g^{\prime}$ and $c i$, and of the lower chord $h^{\prime} e$ and $j e$, the diagonals not stressed being considered omitted from the diagram.

\section{Determination of Stresses. Diagrams Drawn for Com-} bined Loads. CASE I. Parallel Chords. Having plotted the polygon of external forces, we must first determine which diagonal in each panel is stressed under the given system of loads. In the case of parallel chords this may be done as follows (see Fig. 35): 
Using the method of sections, apply the condition of equilibrium, $\Sigma V=0$, to the forces acting on the shaded portion of the truss. The $V$ component stresses in the chord members, $b g$ and he, are each zero, hence the $V$ component stress in the diagonal ( $g h$ or $g^{\prime} h^{\prime}$ ) under stress must be equal and opposite in direction to the $V$ component of the resultant external force. Now it will be seen that this resultant force is represented in the stress diagram by

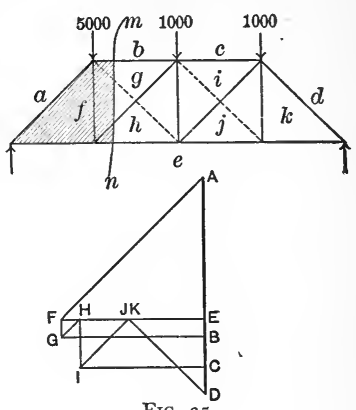

FIG. 35. the letters which lie below and above the panel in question, read in right-handed order, i.e., EB. This order of letters (see stress diagram) is downward, indicating that the resultant external force acts downward, hence the $V$ component diagonal stress must act upwards. Keeping in mind the fact that if a member is in tension the force it exerts upon the shaded portion of the truss acts away from that portion and conversely, we see that if the diagonals are tension members $g h$ is the one under stress, while if they are compression members $g^{\prime} h^{\prime}$ is stressed. Thus by observing whether the resultant external force lying to the left of any panel acts $u p$ or down as indicated in the external force polygon, and knowing whether the diagonals are tension or compression members, we can determine at a glance which diagonal is under stress. The use of the condition $\Sigma V=0$ in determining stresses is called the method of shears.

In Fig. 35 the diagonals are assumed to be tension members, those drawn in full lines being consequently under stress. The dotted diagonals are therefore omitted and the stress diagram constructed in the usual manner. 
CASE 2. Chords not Parallel. In this case the condition of equilibrium, $\Sigma M=0$, can be used, as indicated in the following example, to determine which diagonals are stressed. Having plotted the loads (see Fig. 36 ), the reactions $D E$ and $E A$
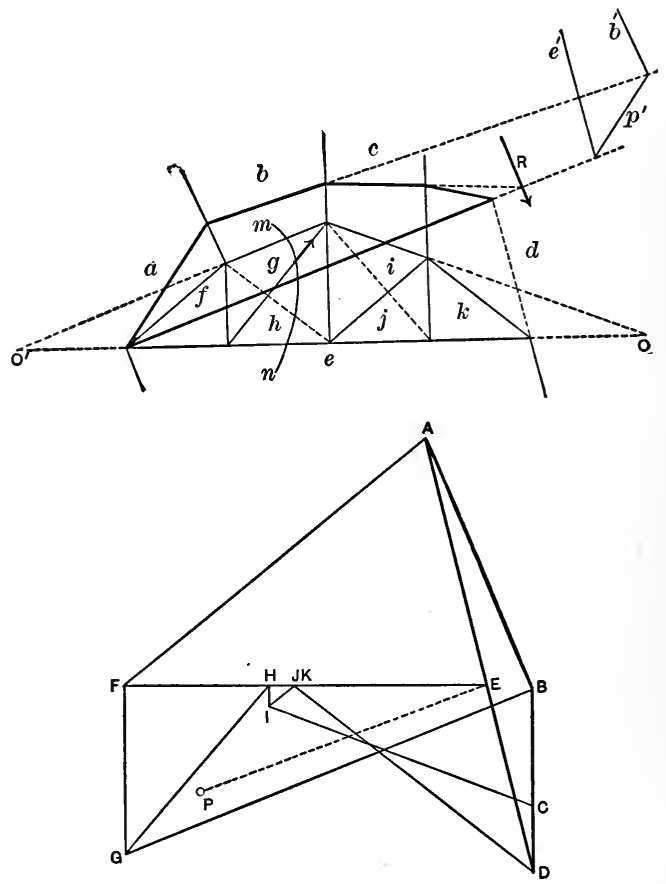

FIG. 36 .

(assumed to be parallel) are determined by drawing a funicular polygon for the joint loads. Now, consider the panel $e b$ and the forces acting on the portion of the truss to the left of the section 
$m n$. The two chord members intersect at $O^{\prime}$. Taking this point as moment axis, the moment of the stresses in the members cut by $m n$ must balance the moment of the external forces acting on the left portion of the truss. The moment of each chord stress being zero, the moment of the stress in the diagonal $g h$ or $g^{\prime} h^{\prime}$ must balance the moment of the resultant external force $E B$. This resultant force must act through the intersection of the strings $e$ and $b$ (see Arts. 8 and 9). The intersection of these strings falls here outside the limits of the drawing, but it is evident from the direction of $E B$ and the general location of the intersection of the two strings $e$ and $b$ that the moment of $E B$ about $O^{\prime}$ would be right-handed; hence the moment of the diagonal stress must be left-handed, i.e., the diagonal $g h$ would be stressed if the diagonals were tension members; and $g^{\prime} h^{\prime}$ if they were compression members. Dealing with the panel $e c$ in the same manner, we find that the moment of the resultant external force $E C=R$, about the axis $O$, is left-handed, hence the moment of the diagonal stress must be right-handed; i.e., $i j$ is stressed if the diagonals are tension members and conversely.

In Fig. 36, the diagonals are assumed to be tension members, those drawn in full lines being stressed. The stress diagram can now be constructed in the usual manner.

If it were necessary to locate the resultant force $E B$, it could be done by Art. 9 (Special Case) as follows: Using $A$ for pole, the strings $e^{\prime}, p^{\prime}$, and $b^{\prime}$ of the special polygon for the forces $E P$ and $P B$ are drawn, the intersection of $e^{\prime}$ and $b^{\prime}$ (not shown) being one point in the line of action of $E B$.

It should be remarked that the diagonals under stress may be determined by trial during the construction of the stress diagram as follows: Omit either diagonal of a panel at random and draw the force polygons, determining from the order of the 
letters the kind of stress in the diagonal used. If this agrees with the stress for which the diagonals were designed, the diagram is correct, otherwise the other diagonal is stressed and the force polygons involved must be re-drawn. This method is unsatisfactory compared with those previously described.

46. Example. The lower chord of the truss, Fig. 2, Plate I, is a circular arc of $185 \mathrm{ft}$. radius. The verticals are equidistant and the diagonals are tension members. Both ends are fixed and the reactions are assumed to be parallel. It is required to determine the stresses in all the members for the following system of loads, viz.: I. A uniformly distributed dead load of 75,000 lbs., the loading ( $18,75 \circ \mathrm{lbs}$.) on the two central panels being divided between the monitor and main roofs as follows: $5000 \mathrm{lbs}$. on the monitor roof and $\mathrm{I} 3,750$ lbs. supported directly by the main roof. 2. A normal wind pressure on the left side, distributed as follows: I7,000 lbs. on vertical surface of main roof; 33,000 lbs. on inclined surface of main roof; $9600 \mathrm{lbs}$. on vertical surface of monitor roof, and I ,, 00 lbs. on inclined surface of monitor roof.

I. Stresses in Monitor Roof. The joint loads and stress diagram are given in Fig. 2 B. The diagonals are assumed to be tension members, $f e$ being evidently the one under stress; $g h$ is therefore omitted. The diagram is constructed beginning at the joint $a b$. The stresses in the members $a h, h g, g f, f e$, and $e d$ evidently act as loads on the main truss and are included in the table of joint loads given below. The computed stress in $g f$ is $5^{\circ}$ lbs. tension.

2. Joint Loads and Reactions for Main Truss. The joint loads for the main truss, resolved into $H$ and $V$ components for convenience in plotting and computing, are given in the following table: 
TABLE of JoInt Loads for MaIn Truss.

\begin{tabular}{|c|c|c|c|c|c|c|c|c|c|c|}
\hline Joints. & $b b_{1}$ & $b_{1} c$ & $c d$ & de & ef & $\mathrm{fg}$ & $g h$ & $h i$ & $i j$ & $j k$ \\
\hline $\begin{array}{l}H \text { loads } . . . . \\
V \text { loads. . . }\end{array}$ & $\begin{array}{c}8500 \\
0\end{array}$ & $\begin{array}{r}1079^{2} \\
9687\end{array}$ & $\begin{array}{r}45^{8} 3 \\
19375\end{array}$ & $\begin{array}{r}45^{8} 3 \\
19375\end{array}$ & $\begin{array}{r}7092 \\
22625\end{array}$ & $\begin{array}{l}93^{8} 3 \\
6 \text { I } 21\end{array}$ & $\begin{array}{l}\cdots \\
14379\end{array}$ & …. & 9375 & \\
\hline
\end{tabular}

Having plotted the joint loads (see Fig. $2 \mathrm{~A}$ ), the reactions $K A$ and $A B$ are determined by drawing a funicular polygon for the resultant joint loads as shown. (In drawing such a polygon. begin with the middle string $f$ or $g$.)

3. Determination of Diagonals Stressed. The method of moments (Art. 45, Case 2) is used to determine which diagonal in each panel is stressed. E.g., in panel $c$, the resultant external force for the portion of the truss to the left of the section $m n$ is $R=A C$, acting through the point of intersection of the strings $a$ and $c$. The point of intersection of the two chord members is not shown but evidently lies to the left of $R$, so that the moment of $R$ about this point is left-handed. The moment. of the diagonal stress must then be right-handed. Therefore, as the diagonals are tension members, the one in full line is. stressed. Investigating each panel in a similar manner, it is found that the diagonals stressed are $z \mathrm{r}, y x, w^{\prime} v^{\prime}, u^{\prime} t^{\prime}, s^{\prime} r^{\prime}, q p$, $o n$, and $m l$. (See notation of Art. 44.)

4. Calculation by Method of Sections. The stress in $g s^{\prime}$ is now calculated by moments as follows: Having previously determined that the dotted diagonal $s^{\prime} r^{\prime}$ is the one stressed, we take as moment axis the intersection of this diagonal and the lower chord member, i.e., the joint $r p$. The computed stress. is $77,330 \mathrm{lbs}$. compression.

It should be observed that it is necessary to know which diagonal is stressed in order to make this calculation.

5. Construction of Stress Diagram. The stress in $g^{\prime}$ is plotted in the diagram and the diagram is completed in the usual 
manner, working in both directions from centre and ends and checking midway. The results are given in the table, Plate I.

It should be noted that no counterbrace is needed in those panels where the same diagonal is stressed under all circumstances.

Problem. In the truss, Fig. 2, Plate I, determine which diagonals are stressed under the action of the dead load alone.

47. Determination of Stresses in Trusses with Counterbracing. SECOND Method. When separate diagrams are constructed

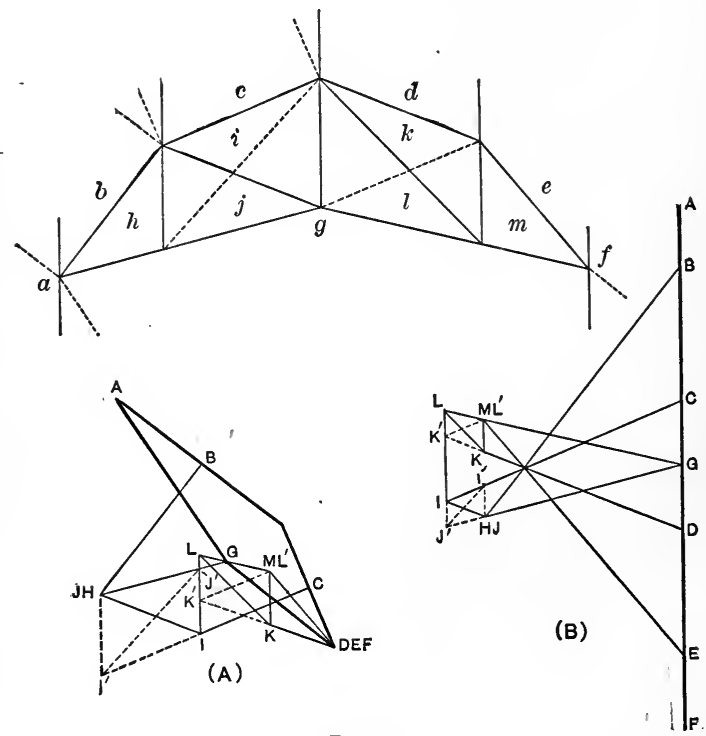

FIG. 37.

for the different kinds of loading (see Art. $3^{6}$ ), the determination of the maximum stresses, where counterbracing is involved, is 
somewhat complicated. A method of procedure is illustrated in the example, Fig. 37. The notation of Art. 44 is employed. The diagonals are assumed to be tension members. The loadings are: I. Dead load; 2. Wind load. Both ends of the truss are fixed, the $H$ reactions being assumed equal. Fig. $37 \mathrm{~A}$ is the diagram for wind load on the left side, Fig. $37 \mathrm{~B}$ is the deadload diagram.

I. In constructing the stress diagrams, one set of diagonals, e.g., those dotted, is omitted, and the force polygons are drawn in the usual manner. The construction is then repeated in the same figure, using the diagonals previously omitted (the additional lines are dotted). The diagrams thus contain the stresses in all the members of the truss, whichever diagonal of each panel is assumed to be stressed. There will be no confusion if the mode of lettering of Art. 44 is employed.

2. The next step is to determine which diagonal of each panel is stressed under each combination of loads, i.e., I. Dead load alone; 2. Dead load and wind on one side.

Dead Load Alone. From Fig. $37 \mathrm{~B}$ we find that the diagonals in tension are $j i$ (full) and $k^{\prime} l^{\prime}$ (dotted). The stresses in the members are therefore lettered in Fig. $37 \mathrm{~B}$ as follows:

$B H, C I, D K^{\prime}, E M, H G, J G, L^{\prime} G, M \mathrm{G}, H J, J I, I K^{\prime}, K^{\prime} L^{\prime}, L^{\prime} M$.

Dead Load and Wind on Left Side. We first determine which diagonal of each panel is stressed by combining the stresses of Figs. $37 \mathrm{~A}$ and $37 \mathrm{~B}$. We thus find that the diagonals in tension are $i^{\prime} j^{\prime}$ (dotted) and $k^{\prime} l^{\prime}$ (dotted). The stresses for this combination of loads are therefore lettered in the two diagrams as follows:

$B H, C I^{\prime}, D K^{\prime}, E M, H G, J^{\prime} G, L^{\prime} G, M G, H I^{\prime}, I^{\prime} J^{\prime}, J^{\prime} K^{\prime}$, $K^{\prime} L^{\prime}, L^{\prime} M$. 
The resultant stresses are therefore found by combining the stresses lettered as above, in the two diagrams.

Dead Load and Wind on Right Side. In this example, this combination of loads need not be dealt with as it is obvious that the maximum stresses in corresponding members on the two sides of the truss will be equal.

3. Having determined the stresses due to the different combinations of loads, the maximum and minimum stresses can be selected as explained in Art. 37 .

\section{Trusses having a Double System of Web Members.} Such a truss may be treated as a combination of two trusses having common chords, but distinct systems of web members. The girder of Fig. $38 \mathrm{~A}$, for example, can be resolved into those shown

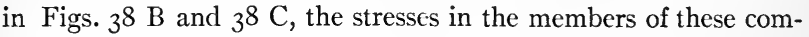
ponent trusses being found in the usual manner. The actual

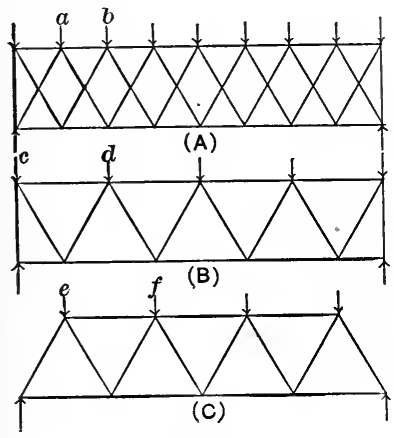

FIG. $3^{8}$. stress in any web member of the original truss is given directly by the diagrams while the stress in any chord segment, as $a b$, is evidently equal to the algebraic sum of the stresses found for $c d$ (Fig. $3^{8}$ B) and $e f$ (Fig. $38 \mathrm{C}$ ). When the given truss can be resolved in more than one way, or when the distribution of loads between the component trusses is uncertain, the problem is

indeterminate.

Fig. 39 is a crescent roof-truss with two systems of web members, as shown by the full and dotted lines respectively. By 
tracing out the force polygons for the different joints it will be seen that the stress diagram can be drawn at once for the complete truss. A suitable system of lettering the interior of the truss is given, the intersections of the diagonals being treated as joints. If the joint I were made to coincide with 2, the portion of its load supported by each component truss would be:

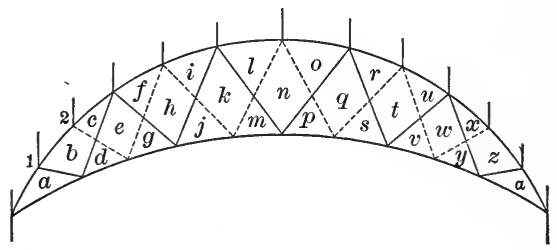

FIG. 39.

uncertain and the problem in this particular would be indeterminate.

49. Double Diagonal Bracing. In the case of counterbraced panels (see Fig. $33 \mathrm{~N}, \mathrm{O}, \mathrm{P}, \mathrm{Q}$ ), the two diagonals are assumed not to be in action for the same loading; both being designed for the same kind of stress. When the diagonals are designed to act simultaneously (one being in tension while the other is in compression), the truss can be resolved into two trusses having common chords and verticals but distinct systems of diagonals. Each joint would belong to both component trusses, and the division of its load between the two trusses would be uncertain. One way of dealing with this case is to assume that the load at each joint is divided equally between the two trusses. The stresses in the verticals and chords would be the algebraic sum of those found for the separate trusses. The results of this method are evidently liable to err in the wrong direction. 
Maw 24

\section{§ 5. Three-hinged Arch.}

50. Definition. A three-hinged arch consists of two arched ribs hinged at the crown and abutments (Fig. 40). The outward thrust at the ends may be resisted by the abutments or by a tierod joining the ends. The ribs may be braced or solid.

5I. Determination of Reactions of Hinges. The reactions are assumed to act through the centres of the hinges. The reactions of the hinge at the crown on the two half-ribs must be evidently equal and opposite. Let $a b$ (Fig. 40) represent the line of action of the resultant load supported by the left half-rib, the right half being assumed to be unloaded. The reactions at $O^{\prime}$ and $O^{\prime \prime}$ must be equal and act along the line $O^{\prime \prime} O^{\prime}$. The three forces acting on the left half must, for equilibrium, intersect at a common point. This condition determines $O n$ to be the direction of the reaction at $O$.

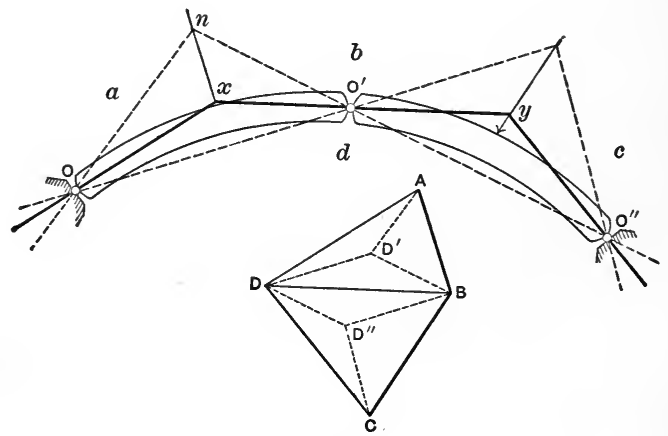

FIG. 4 .

The magnitudes of these reactions can now be found by constructing the triangle of forces $A B D^{\prime} . B D^{\prime}$ is the magnitude 
of the reaction at $O^{\prime \prime}$, and of the two equal and opposite reactions at $O^{\prime} . \quad D^{\prime} A$ is the magnitude of the reaction at $O$.

The directions and magnitudes of the reactions at $O, O^{\prime}$, and $O^{\prime \prime}$, for any resultant load $b c$ on the right half-rib, are found in a similar manner, the triangle of forces being $B C D^{\prime \prime}$. When both sides are loaded, the reaction at either hinge is evidently the resultant of the reactions due to the loads taken separately. Combining the two separate reactions for each of the hinges by the triangle of forces, we find the resultant reactions for the right half-rib to be $C D$ and $D B$ at $O^{\prime \prime}$ and $O^{\prime}$ respectively; and for the left half-rib, $B D$ and $D A$ at $O^{\prime}$ and $O$ respectively.

The lines of action of these reactions are $O x, x O^{\prime} y, y O^{\prime \prime}$, drawn parallel to $D A, D B$, and $D C$ respectively. These lines should intersect on $a b$ and $b c$ as shown, if the construction is accurate.

In the case of a symmetrical arch, symmetrically loaded, the reactions at the crown will evidently be horizontal. The reactions of the end hinges can then for this case be found directly; the points $x$ and $y$ being determined by the intersections of a horizontal line through $O^{\prime}$ with the resultant loads.

Fig. 40 can now be interpreted as follows: The point $D$ can be taken to be the pole, and the lines $O x, x y, y O^{\prime \prime}$ the strings of a funicular polygon for the given loads. The reactions of the hinges can, then, be determined by constructing a funicular polygon for the loads, such that the three strings will pass through the hinges. The strings will be the lines of action of the reactions, and the lengths of the corresponding rays their magnitudes.

Instead of using the resultant loads, the actual loads may be employed in applying this method. In this case it is only necessary to construct a funicular polygon for the given loads, such that the three limiting strings will pass through the three hinges. (For methods, see Arts. 23 and 24.) 
52. Determination of Reactions. Alcebraic Solution. In order to secure greater accuracy, it may be desired to determine by calculation the position of the pole of the required funicular polygon. This is done by calculating the reaction at $O^{\prime}$, since the ray $D B$ (Fig. 40) or $P D$ (Fig. 4I) represents this reaction.

Let $R,+R$ be the equal and opposite reactions at $O^{\prime}$ (Fig. 4I). These are resolved into horizontal $(H)$ and vertical $(V)$ components. Taking moments, about $O$, of the forces acting on the left half, we have $H \cdot b^{\prime}-V \cdot a^{\prime}=\Sigma W^{\prime} x^{\prime}$, in which $W^{\prime}$ represents any load, and $x^{\prime}$ its arm. Similarly, the moments about $O^{\prime \prime}$ of the forces acting on the right half give the equation $H \cdot b^{\prime}+V \cdot a^{\prime}=\Sigma W x$. By solving these two equations, we determine the values of $H$ and $V$, and consequently $R$. This value

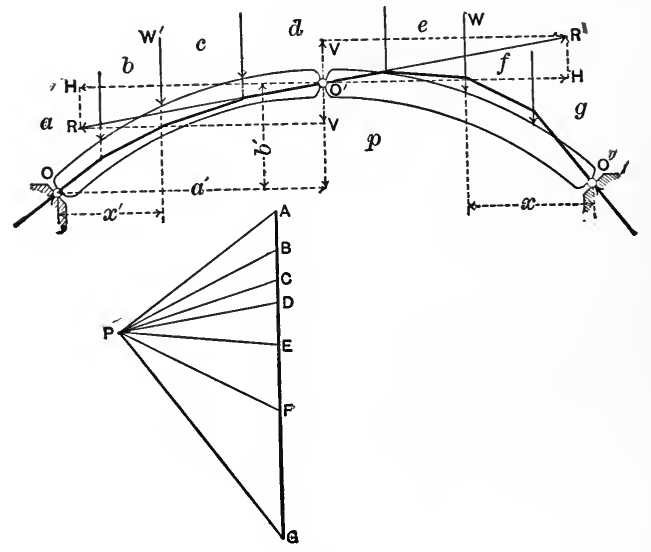

FIG. 4I.

of $R$, laid off in the proper direction from the point $D$ of the force polygon, locates the pole $P$ of the required funicular polygon. $P A$ and $P G$ are then the reactions at $O$ and $O^{\prime \prime}$ respectively. 
It is to be noted that $P A D$ and $P D G$ are the polygons of external forces for the two half-ribs.

Problem. The semicircular arch (Fig. 42) hinged as shown, is loaded with a dead load of 8000 pounds uniformly distributed over the roof, and also with a wind load of 8000 pounds on the right side. Find the reactions of the hinges: (I) graphically; (2) by calculation as explained. Also draw a funic-

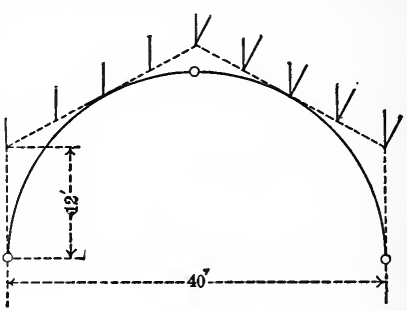

FIG. 42. ular polygon for these loads, to pass through the three hinges.

53. Determination of Stresses in Braced Arches. The determination of the stresses in three-hinged braced arches will be illustrated by the following example. Fig. 3, Plate II, represents half of a symmetrical three-hinged arch. The outer inclined chord is divided by the joints into 6 divisions of $5 \mathrm{ft}$. each and one of $8.1 \mathrm{ft}$. The member $\tau, 4$ is drawn so as to make the adjacent segments of the inner chord equal, the other corresponding members being perpendicular to the outer chord. The arch is loaded with a dead load uniformly distributed over the outer chord, together with a wind load on the right side. The wind pressure on the yertical sides of the building is not supported by the arches. It is required to determine the stresses in the windward rib. The diagonals are assumed to be tension members. The joint loads (pounds) are as follows:

\begin{tabular}{c|c|c|c|c|c|c|c|c|c}
\hline Joint. & $a b$ & $b c$ & $c d$ & $d e$ & ef & $f g$ & $g h$ & $h$ & Total. \\
\hline Dead Load. .... & $\mathbf{1} 620$ & 2620 & 2000 & 2000 & 2000 & 2000 & 2000 & $\mathbf{1 0 0 0}$ & $\mathbf{1 5 2 4 0}$ \\
Wind Load. .... & 2430 & 3930 & 3000 & 3000 & 3000 & 3000 & 3000 & $\mathbf{1} 500$ & $\mathbf{2 2 8 6 0}$ \\
\hline
\end{tabular}


I. Determination of Reactions of Hinges. The resultant dead and wind loads, $I K$ and $K A$ (Fig. $3 \mathrm{~A}$ ), are plotted and their resultant $(I A)$ is subdivided into the joint loads. The $H$ and $V$ reactions of the middle hinge, calculated by the method of Art. 52, are: $H=\mathrm{I}_{4}, 860$ lbs.; $V=9900$ lbs. These are plotted (see Fig. $3 \mathrm{~A}$ ), thus locating the pole $P$. A funicular polygon for the resultant dead and wind loads is now constructed by drawing the string (a) through the end hinge, parallel to $P A$, and the string (i) through the middle hinge, parallel to $P I$; when it is found that the closing string $(k)$ is parallel to $P K$, thus checking the location of the pole. The reactions of the hinges are thus $P I$ and $A P$, and the polygon of external forces is PIHGFEDCBAP.

2. Line of Pressure. The funicular polygon for the resultant joint loads is now drawn (see Fig. 3). The significance of this polygon should be noted. Beginning at the left end, the string $i$ is the reaction on the hinge, its magnitude being $P I$; the string $h$ is the line of action of the resultant of this reaction and the load at the apex, its magnitude being $P H$; the string $g$ is the line of action of the resultant of the reaction and the loads $i h$ and $h g$, its magnitude being $P G$, etc. Thus it is seen that any string of the funicular polygon for the joint loads, drawn through the hinges, represents the line of action of the resultant external force acting on the portion of the rib to either side of the panel in question; the magnitude of this force being represented by the corresponding ray.

This funicular polygon is called the Line of Pressure, since its strings represent the lines of action of the resultant pressure on the corresponding panels of the arch.

3. Determination as to which Diagonals are Stressed. The methods of Art. 45 are employed to determine which diagonals are stressed, the application of these methods being based on the preceding paragraph.

Take, for example, panel $g$, where the chords are parallel. 
The resultant external force, $R$, to the left of the section $m n$ is $P G$, acting along the string $g$ as shown. The component of this force at right angles to the chords evidently acts upwards; the corresponding component of the diagonal stress must then act downwards (method of shears); hence if the diagonals are tension members, the one drawn full $(16-17)$ is the one stressed.

Again, take the panel $c$, where the chords are not parallel. The resultant external force, $R^{\prime}$, to the left of $m^{\prime} n^{\prime}$, is $P C$ acting along the string $c$ as shown. The moment of this force about the intersection (not shown) of the chord members is evidently right-handed; hence the moment of the diagonal stress must be left-handed; therefore, the diagonals being tension members, the one drawn full (8-9) is stressed. In Fig. 3 the diagonals stressed are drawn in full lines.

4. Calculation of Stress in c-8 by Moments. Having previously found $8-9$ to be the diagonal stressed in this panel, we take the moment axis at the intersection of this diagonal and the inner chord member, i.e., at the joint $o$. In calculating the resultant moment of the external forces to the left of $m^{\prime} n^{\prime}$, about $o$, we may use the $H$ and $V$ component reactions at the middle hinge and the stated joint loads to the left of $m^{\prime} n^{\prime}$, or we may take the moment of their resultant as represented by the string $c$ and the ray $P C$. In the latter case we would find the magnitude of this resultant by scaling off $P C$ from the stress diagram, and the moment arm by scaling off the perpendicular distance from $o$ to the string $c$. This moment divided by the perpendicular distance from $o$ to $c-8$ would give the stress in $c-8$. Its value is $35^{2} 50 \mathrm{lbs}$. compression.

5. Completion of Stress Diagram. We plot the computed stress in $c-8$ and complete the diagram in the usual way, checking it on the members $4-5$ and $\mathrm{I}_{2}-\mathrm{I} 4$ (marked $c k$ in the stress diagram). In an arch of many members more sections than one 
should be taken to secure sufficient accuracy. In the preceding example counterbracing was introduced in order to indicate the use of the line of pressure in determining which diagonals are stressed. It is advisable to draw the line of pressure in any case.

Problem. Determine the stresses in the leeward rib of the arch of the preceding example, also the stresses for dead load alone. Find the maximum and minimum stresses in all the members for dead and wind loads.

Figure 43 represents one of the arches supporting the trainshed roof of the Philadelphia and Reading Terminal Railway. The diagonals are tension members. ${ }^{1}$

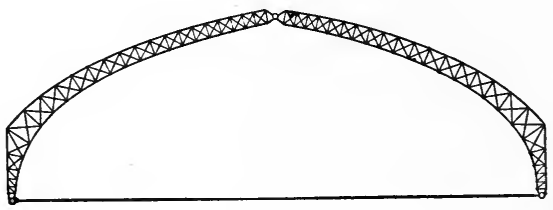

FIG. 43.

54. Three-hinged Arch. Solid Ribs. Determination of Stresses. Let Fig. 44 represent such an arch, the line of pressure and the force polygon for the given system of loads being drawn. The resultant external force acting to the right of any crosssection $n$ is $D P=F$, its line of action being the corresponding string. Apply at $n$, the centre of gravity of the cross-section, opposite forces equal and parallel to $F$, and resolve one of these forces into $F^{\prime}=D x$ and $F^{\prime \prime}=x P$, respectively perpendicular and parallel to the section. The original force and $-F$ form a couple which causes a bending stress at the section, the bending moment being equal to the moment of the original force about the centre of

${ }^{1}$ See Trans. Am. Soc. C. E., Aug., 1895. 
gravity of the section. In addition, $F^{\prime}$ is the direct compression stress and $F^{\prime \prime}$ the shearing stress at the section.

We thus see that the stress at the section is made up of:

I. A bending stress, the bending moment $(M)$ being the moment of the resultant external force on either side of the section, taken about the centre of gravity of the section. If we let $I=$

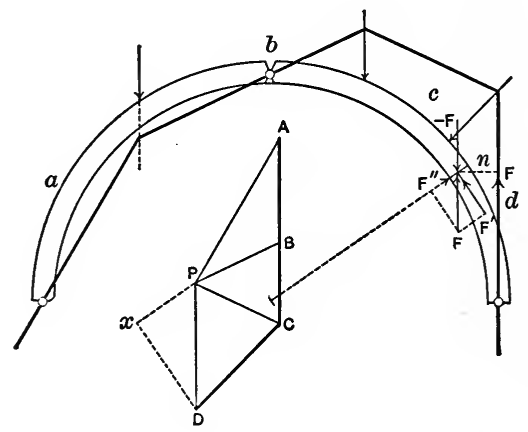

Fig. 44.

moment of inertia of the section about the neutral axis through the centre of gravity, and $y$ the distance from this neutral axis to the most compressed fibre, we have for the maximum intensity of the bending (compression) stress

$$
f_{1}=\frac{M}{I} y_{\bullet} \quad \bullet \cdot \bullet \cdot \bullet \cdot \bullet \cdot
$$

2. A uniformly distributed compression stress, $F^{\prime}=D x$, whose intensity is

$$
f_{2}=\frac{F^{\prime}}{A} ; \quad \bullet \cdot \bullet \cdot \bullet \cdot \bullet \cdot
$$


$A=$ area of section. Combining (I) and (2), the maximum intensity of the compression stress will be

$$
f=f_{1}+f_{2}=\frac{M}{I} y+\frac{F^{\prime}}{A} . . . . . .
$$

3. A transverse shearing stress, $F^{\prime \prime}=x P$. This last stress will usually be comparatively small in the case of arches.

\section{Bending Moments Proportional to Vertical Intercepts.} In the case of vertical loads, the bending moments are proportional to the vertical intercepts between the line of pressure and the centre line of the arch ring.

Proof: Let $R S$ (Fig. 45) be a portion of the centre line of an

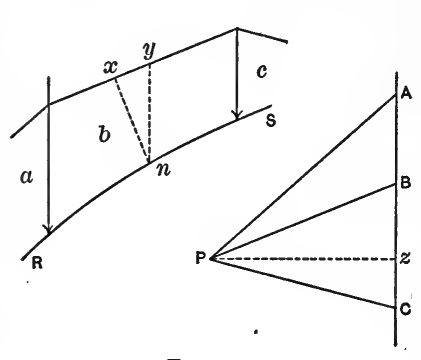

FIG. 45. arch, the corresponding portions of the force diagram and A line of pressure being shown. Let $n$ be any section of the arch, $n x$ being drawn perpendicular to the string $b$, and $n y$ vertical. The triangles $n x y$ and $P z B$ are similar, hence $\frac{n x}{n y}=\frac{P z}{P B} ; \therefore P B \cdot n x=P z \cdot n y$.

But $P B \cdot n x=$ bending moment at $n$ (see Art. 54 ), hence $P z \cdot n y$ also equals the bending moment at $n$. As the pole distance $(P z)$ is constant for vertical loads, the moments are proportional to $n y$, the vertical intercepts. Q.E.D.

Problem. Given a symmetrical three-hinged semi-circular arch of $20 \mathrm{ft}$. span between end hinges. The hinges intersect the centre line of the arch ring. The arch is loaded with a vertical load of ro,ooo lbs. $5 \mathrm{ft}$. to the left of the crown hinge, and with a vertical load of $2000 \mathrm{lbs}$. $6 \mathrm{ft}$. to the right of the crown 
hinge. The cross-section is an $I$ section of equal flanges, Io inches deep, $5 \mathrm{sq}$. inches section, moment of inertia $=150$ (inches). Find the maximum intensity of the compression stress.

\section{\$6. Bending Stresses. Sway-bracing.}

56. Conditions under which Bending Stresses occur. In order that no bending stresses may occur in the members of a frame, the following conditions must, in general, be fulfilled: I. The centre lines of the members must be straight and intersect at a common point at the various joints. 2. External forces. must not act at any other points than the joints. 3. The various. members must be free to turn at the joints. If these conditions. are not fulfilled, bending stresses of greater or less magnitude and definiteness will occur and should be taken into account.

Some of the more prominent cases in which such bending; stresses occur are:

I. Curved members. 2. External forces acting at other points than the joints. E.g., when the purlins are supported on the upper chord of a roof-truss at other points than the joints. 3. Incomplete or defective trusses, i.e., trusses in which the members are either insufficient in number or improperly placed, so' that their resistances to tension and compression are inadequate: to secure stability. 4. Columns in buildings. 5. Centre lines of members forming a joint, not intersecting at a common point, etc.

Maximum Intensity of Stress. The determination of the maximum intensity of the combined bending and direct (tension or compression) stress is similar in all cases to that given in Art. 55 . If we let $P=$ total direct stress at any section of a member, $A=$ area of section, $M=$ bending moment at section, $I=$ moment of inertia of section about neutral axis through centre of gravity, 
and $y=$ distance from this neutral axis to the most stressed fibre; we have, for the maximum intensity of the combined stress,

$$
f=\frac{P}{A}+\frac{M}{I} y \text {. }
$$

The following Articles give solutions of some special cases in which bending stresses occur:

57. Purlins supported at Other Points than the Joints. Example. The truss, Fig. $46 \mathrm{~A}$, is loaded with a uniformly distributed vertical load of 9000 lbs. The purlins divide the upper chord into six equal parts; thus the load at each intermediate
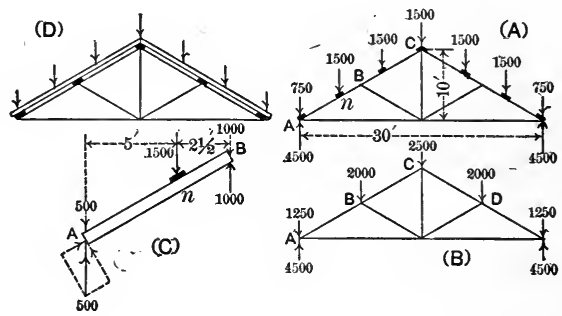

FIG. 46.

purlin point is $1500 \mathrm{lbs}$., and at each end point $75^{\circ} \mathrm{lbs}$. It is required to determine the stresses in the frame, including the maximum intensity of compression in the different segments of the upper chord, its cross-section being $8^{\prime \prime}$ wide by $10^{\prime \prime}$ deep.

Consider the portion $A B$ of the upper chord and the load at $n$. The components of this load at $A$ and $B$ are $500 \mathrm{lbs}$. and Iooo lbs. respectively. Therefore apply at these points equal and opposite forces of $500 \mathrm{lbs}$. and rooo lbs. as shown in Fig. $46 \mathrm{C}$. These added forces will neutralize each other and hence not 
affect the stresses, so that the system of forces given in Fig. $46 \mathrm{C}$ are the equivalent of the $1500 \mathrm{lbs}$. load at $n$. This system of forces may be separated into two systems, namely: I. The downward forces of $500 \mathrm{lbs}$. and $1000 \mathrm{lbs}$. at $A$ and $B$ respectively. These cause direct stresses in the members of the truss. 2. The load at $n$ and the upward forces at $A$ and $B$, these latter corresponding to the reactions of a beam $A B$, loaded at $n$. This. system of forces (shown by full lines in Fig. $46 \mathrm{C}$ ) will cause bending stresses, also direct compression and shearing stresses in $A B$.

The preceding analysis leads to the following solution: (I) Resolve each load into components at the adjacent joints, thus obtaining the joint loads of Fig. 46 B. Determine the direct stresses in the members of the truss, due to these joint loads, by constructing a stress diagram in the usual way.

(2) Treat each segment of the upper chord as an oblique beam supported at the ends (joints) and loaded at the purlin points, calculating the beam stresses in the usual way.

(3) Combine the stresses found in (I) and (2).

(The following conception of the case may be of service in making this method of solution clearer. If the loads of Fig. $46 \mathrm{~A}$ were supported as shown in Fig. $46 \mathrm{D}$, instead of being applied directly to the upper chord, there would be bending stresses in the jack-rafters, and direct stresses only, in the upper chord. In this case the forces acting at the joints of the upper chord and those acting on the jack-rafters, together with the resulting stresses in these members, would be determined as indicated in (I) and (2) respectively.

In Fig. $46 \mathrm{~A}$ the upper chord alone serves the same purpose as the jack-rafters and upper chord together in Fig. $46 \mathrm{D}$, so that if the cross-sections of jack-rafters and upper chord of Fig. $46 \mathrm{D}$ were each equal to the cross-section of the upper chord of 
Fig. $46 \mathrm{~A}$, the stresses in the latter would be equal to those found by combining the stresses existing in both jack-rafters and upper chord of Fig. 46 D.

The case may be stated otherwise as follows: Combining the external forces acting on the jack-rafters and upper chord of Fig. $46 \mathrm{D}$, we obtain those given in Fig. $46 \mathrm{~A}$. Therefore, if we combine the stresses in the jack-rafters and upper chord of Fig. $46 \mathrm{D}$, we will obtain those existing in the upper chord of Fig. $46 \mathrm{~A}$, provided, of course, that the cross-sections are identical in each instance.)

Solution. I. By drawing a stress diagram (not shown) for the joint loads of Fig. $46 \mathrm{~B}$, we find the stress in $A B$ to be $5^{8} 5^{\circ}$ lbs. compression, and in $B C$ to be 4050 lbs. compression.

2. Treating $A B$ (see Fig. $46 \mathrm{C}$ ) as a beam loaded with $I_{500}$ lbs. at $n$, we find that the maximum bending moment (at $n)=$ $500 \times 5 \times 12=30,000$ inch-lbs. Also, by resolving the reaction at $A$ into components along and at right angles to the beam, we find an additional direct compression stress of $278 \mathrm{lbs}$. in the part of $A B$ below $n$, due to the obliquity of the loading.

3. We thus find the maximum direct stress (compression) in $A B$ to be $5850+278=6$ I $28 \mathrm{lbs}$., and the maximum bending moment to be 30,000 inch-lbs. Substituting these values in the formula of Art. 56, we have, for the maximum intensity of compression in $A B, f=\frac{6128}{80}+\frac{30,000 \cdot 5}{667}=302 \mathrm{lbs}$. per square inch.

In a similar manner we find the maximum intensity of compression in $B C$ to be $283 \mathrm{lbs}$. per square inch.

It will be observed that the additional stress due to the obliquity of the loading is small.

Problem. Given a triangular truss of $20 \mathrm{ft}$. span and $6 \mathrm{ft}$. rise, loaded with a uniformly distributed vertical load of 8000 lbs. applied directly to the rafters. The rafters are wooden beams 
4 in. by ro in. section. Find the maximum compression stress per square inch in these members.

58. Trussed Beam. Example. The trussed beam $D E$ (Fig. $47 \mathrm{~A}$ ) is loaded uniformly. It is required to find the stresses in the members.

As a beam, $D E$ is supported at three equidistant points and loaded uniformly. The reaction of the middle support in such a case (see Strength of Materials) is $\frac{5}{8} W$, and of each end support ${ }_{16}^{3} W$. Therefore apply equal and opposite forces of $\frac{5}{8} W$ at the middle and ${ }_{1 \frac{3}{6}} W$ at each end as shown by the dotted lines. The forces of Fig. $47 \mathrm{~A}$ can now be separated into two bal-

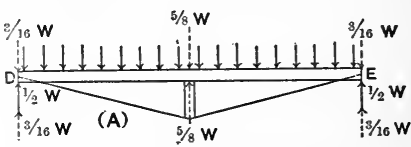
anced systems, shown in Figs. $47 \mathrm{~B}$ and $47 \mathrm{C}$ respectively. Fig. 47 B represents the forces acting on

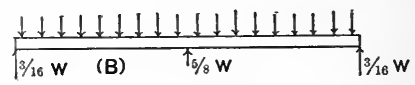
$D E$ as a beam, the maximum fibre stress being found by the methods of "Strength of Materials." Fig. $47 \mathrm{C}$ represents the forces acting at the joints, the resulting tension and compression stresses being found by constructing a stress diagram. Finally, the maximum intensity of the stress in $D E$ is found by combining the stresses obtained from Figs. $47 \mathrm{~B}$ and $47 \mathrm{C}$ in the same manner as in Art. 57 .

59. Incomplete Trusses. Frames of this character are usually subjected to bending as well as direct stresses. The solution of a simple case follows. 
If the frame of Fig. 48 were loaded symmetrically at the joints, no bending stresses would occur, and the direct stresses would be found from a stress diagram in the usual way.

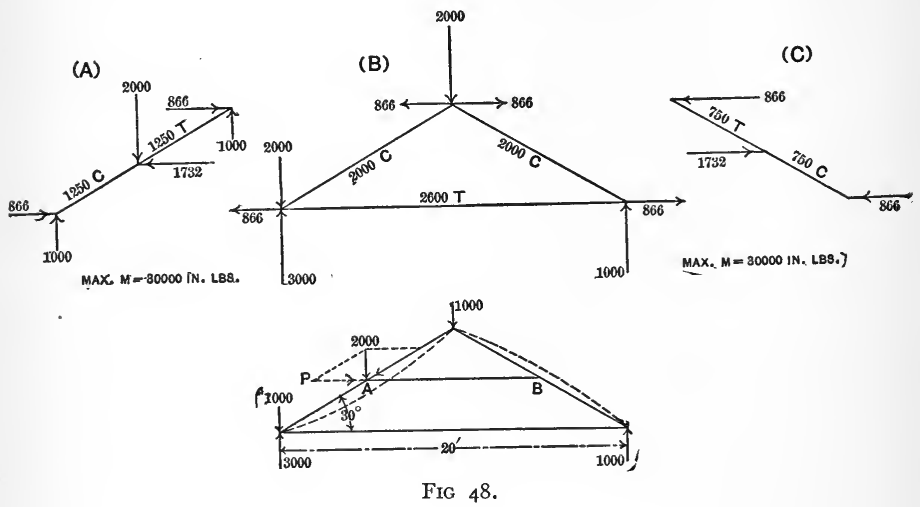

Under an unsymmetrical load, however, such as given in Fig. 48, the rafters would evidently deflect equally in the manner indicated by the dotted lines.

In order to determine the stress in $A B$, resolve the load at $A$ into a horizontal component $P$, and one acting along the rafter. We thus obtain $P=2000 \tan 60^{\circ}=3464 \mathrm{lbs}$.

Let $x=$ compression stress in $A B$. Now, since the deflections of the two rafters are equal, the horizontal pressures at $A$ and $B$ must be equal.

$$
\therefore P-x=x ; \quad \therefore x=\frac{1}{2} P=\mathrm{I} 732 \mathrm{lbs} \text {. }
$$

The frame can now be treated as a triangular truss loaded at $A$ with $2000 \mathrm{lbs}$. (vertical) and $\mathrm{5} / 32 \mathrm{lbs}$. (horizontal) acting 
to the left; and loaded at $B$ with $173^{2}$ lbs. (horizontal) acting to the right, in addition to the joint loads of 1000 lbs. each at apex and left support. Following the method of Art. 57, these loads can now be separated into the systems of loads shown in Figs. $48 \mathrm{~A}, \mathrm{~B}$, and $\mathrm{C}$.

The stresses due to the joint loads (Fig. $48 \mathrm{~B}$ ) are determined from a stress diagram. They are given in the figure.

Solving Fig. 48 A, we find additional direct stresses, due to the obliquity of the loads, of 1250 lbs. compression in the lower half, and I250 lbs. tension in the upper half of the rafter. We have also a maximum bending moment in the rafter of 3nooo inch lbs.

Solving Fig. $48 \mathrm{C}$, we find additional direct stresses of $75^{\circ}$ lbs. compression in the lower half, and $75^{\circ}$ lbs. tension in the upper half of this rafter; also a maximum bending moment of 30000 inch-lbs.

Combining the stresses in these three diagrams, we obtain the following results:

Max. direct stress in left-hand rafter $=3250$ lbs. C. . . . . (I)

" " " " right-hand rafter $=2750$ lbs. C. • • . (2)

Stress in lower chord $=2600$ lbs. T. . . . . . . . . . (3)

Max. bending moment in left-hand rafter $=30000$ in.-lbs. . (4)

" " " " right-hand rafter $=30000$ in.-lbs. . (5)

Finally, (the rafters are assumed to be $4^{\prime \prime}$ wide by $10^{\prime \prime}$ deep) we have, combining (I) with (4) and (2) with (5):

Max. intensity of compression in left-hand rafter

$$
=\frac{3250}{40}+\frac{30000 \cdot 5}{333}=53 \mathrm{I} \mathrm{lbs.} \mathrm{per} \mathrm{sq.} \mathrm{inch.}
$$


Max. intensity of compression in right-hand rafter

$$
=\frac{2750}{40}+\frac{30000 \cdot 5}{333}=5 \text { I9 lbs. per sq. inch. }
$$

6o. Trusses supported by Columns. (For examples see Fig. 33 G, H, and P, also Figs. 49, 52, and 54.) The entire frame, including columns, will be treated together, the points of support being at the bases of the columns.

There are two general cases, namely: I. When the columns are rigidly attached to substantial foundations so that they may be considered fixed in direction at the base. 2. When fastenings and foundations are insufficient for this, in which case the columns are assumed to be hinged at the base.

If the sides of the building are masonry of sufficient strength, the frame will be required to resist wind pressure on the roof surface only. If, however, the sides are of wood, corrugated iron, etc., supported by the columns, the frame must resist the wind pressure acting on the sides as well as roof of the building.

Under the action of the wind or other non-vertical loads the columns will be subjected to bending stresses. Special cases are discussed in the following articles.

6r. Case I. Columns Hinged at Base. The Joints of the Frame may be subjected to Any System of Loads; the Columns, in addition, being loaded at Other Points than the Joints. In order to avoid unnecessary complication, the additional column loading will be assumed to be a horizontal wind load, $W$, uniformly distributed over the left-hand column (Fig. 49). The method used in deriving the formulas which follow is applicable to any other system of column loads. 
Let Fig. 49 represent the frame, knee-braces, $d, f$, and $d^{\prime}, f^{\prime}$ connecting the columns and truss. The joint loads are not shown, the additional column loading $(W)$ only being represented. The notation of Art. 28 will be used as far as it is applicable, $\Sigma H$ and $\Sigma V$ representing the horizontal and vertical component loads (including column loading).

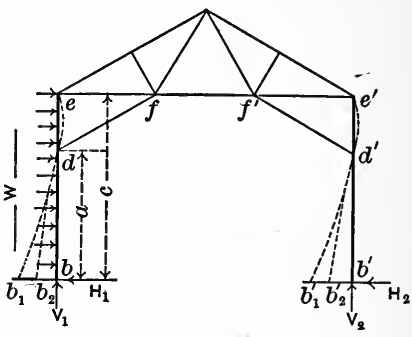

FIG. 49 .

Assumptions. As in case of trusses supported on masonry walls (see Art. 28), this solution must be based on assumptions; those which appear to be as reasonable as any, and which will be employed here, being that the truss is comparatively rigid, the distances $e-e^{\prime}, d-d^{\prime}$, and $b-b^{\prime}$ remaining unchanged, while the columns bend as shown by the dotted lines $e, d, b_{1}$, and $e^{\prime}, d^{\prime}, b_{1}^{\prime}$, being assumed free to turn at the joints $e, d, b$, and $e^{\prime}, d^{\prime}, b^{\prime}$. It follows from these assumptions that the deflections of the two columns at $d$ and $d^{\prime}$, relative to straight lines joining their ends, will be equal.

Separation of the Loads into: I. Those causing beam stresses in the columns. 2. Those causing direct stresses in the frame.

In order to make clear the manner of separating the loads, we will employ a conception similar to that stated in Art. 57 . It is as follows: The left-hand column (Fig. 49) is united to the frame at the points $e$ and $d$. This column is subjected to both direct and bending stresses. Now let us imagine that a separate piece (Fig. 50 B), of the same cross-section as the column, and connected with the frame by rods $e, e$ and $d, d$ be employed to resist the bending stresses (the right-hand column being simi- 
larly treated), so that the columns of Fig. $50 \mathrm{~A}$ will be subjected to direct stresses only.

Under these circumstances the forces acting in Fig. $50 \mathrm{~B}$ will include: $\mathrm{I}$. The horizontal reaction $H_{1}$ at the base; 2. The wind pressure $W ; 3$. The reactions $R_{1}$ and $S_{1}$ of the connecting rods. These forces evidently cause bending stresses. Similar

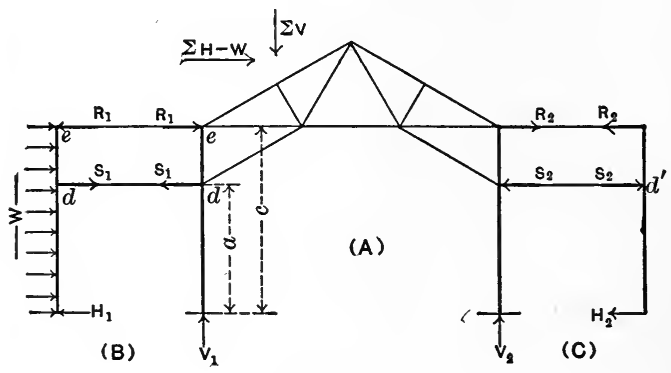

FIG. 50.

conditions exist at the right-hand end, except that there is no wind load.

The forces acting on the frame (Fig. 50 A) will include: I. The vertical reactions $V_{1}$ and $V_{2} ; 2$. The joint loads whose $H$ and $V$ components are represented by $\Sigma H-W$ and $\Sigma V$ respectively; 3 . The reactions $R_{1}, S_{1}$ and $R_{2}, S_{2}$ of the rods. This loading evidently causes direct stresses only, in the members of the frame.

If now we combine the pieces of Figs. $5 \circ \mathrm{B}$ and $5 \circ \mathrm{C}$ with the left- and right-hand columns, respectively, of Fig. $5 \circ \mathrm{A}$, the equal and opposite reactions, $R_{1}, S_{1}, R_{2}$, and $S_{2}$ will neutralize each other so that the combined external forces will be identical with those of Fig. 49. This being the case, we can, by combining in the usual manner the stresses existing in the pieces of Figs. $5 \circ \mathrm{B}$ and 
$50 \mathrm{C}$ with those of Fig. $50 \mathrm{~A}$, obtain the actual stresses in the frame.

I. Determination of the Values of $R_{1}, R_{2}, S_{1}, S_{2}, H_{1}, H_{2}, V_{1}$, and $V_{2}$. Applying the general conditions of equilibrium $(\Sigma H=o$; $\Sigma V=o ; \Sigma M=o$ ) to the forces of Figs. $5 \circ \mathrm{A}, \mathrm{B}$, and $\mathrm{C}$ we have:

From Fig. 5० B. $H_{1}=S_{1}+W-R_{1}$

$$
(\Sigma H=o) \quad \cdot .
$$

$$
H_{1} \cdot c=\frac{W \cdot c}{2}+S_{1}(c-a) \quad(\Sigma M=o) \quad \text {. }
$$

From Fig. 50 C. $H_{2}=S_{2}-R_{2}$

$$
(\Sigma H=0) \text {. } .
$$

$$
H_{\circ} \cdot c=S_{2}(c-a)
$$$$
(\Sigma M=0) \quad \cdot \quad
$$

From Fig. 50 A. $S_{1}+S_{2}=R_{1}+R_{2}+\Sigma H-W(\Sigma H=0) \quad$. (5) Also: Deflection at $d$ (Fig. 5० B) $=$ deflection at $d^{\prime}$ (Fig. 50 C) . (6)

To find the deflection at $d$, we have from the ordinary formulas for deflection of beams, treating Fig. $50 \mathrm{~B}$ as a beam supported at the ends and loaded with $S_{1}$ and $W$ :

Deflection at $d$ due to $S_{1}=\frac{S_{1} a^{2}(c-a)^{2}}{3^{c} E I}$;

Deflection at $d$ due to $W=\frac{W a(c-a)}{24 c E I}\left(c^{2}+c a-a^{2}\right)$.

Adding, we have:

Total deflection at $d=\frac{S_{1} a^{2}(c-a)^{2}}{3^{c E I}}+\frac{W a(c-a)}{24 c E I}\left(c^{2}+c a-a^{2}\right)$.

Similarly:

Total deflection at $d^{\prime}=\frac{S_{2} a^{2}(c-a)^{2}}{3^{c E I}}$...$\cdot$. $\cdot$. 
Equating (7) and (8) and reducing, we have

$$
S_{2}-S_{1}=\frac{W\left(c^{2}+c a-a^{2}\right)}{8 a(c-a)} \text {. . . . . }
$$

Adding (2) and (4) and substituting $H_{1}+H_{2}=\Sigma H$ (Art. 28) we have

$$
S_{2}+S_{1}=\frac{(\Sigma H) \cdot c}{c-a}-\frac{W \cdot c}{2(c-a)} \cdot \cdot \cdot \cdot
$$

Solving (9) and (10), we have

$$
\begin{aligned}
& S_{1}=\frac{(\Sigma H) \cdot c}{2(c-a)}-\frac{W\left(c^{2}+5 c a-a^{2}\right)}{\mathrm{I} 6 a(c-a)} ; \quad . . \\
& S_{2}=\frac{(\Sigma H) \cdot c}{2(c-a)}+\frac{W\left(c^{2}-3 c a-a^{2}\right)}{\mathrm{I} 6 a(c-a)} .
\end{aligned}
$$

Substituting (II) and (I 2) in (2) and (4), we have

$$
\begin{aligned}
& H_{1}=\frac{\Sigma H}{2}-\frac{W\left(c^{2}-3 c a-a^{2}\right)}{\mathrm{I} 6 c a} ; . . . \\
& H_{2}=\frac{\Sigma H}{2}+\frac{W\left(c^{2}-3 c a-a^{2}\right)}{\mathrm{I} 6 c a} . . .
\end{aligned}
$$

Substituting (II) and (I3) in (I), and (I2) and (I4) in (3), we have

$$
\begin{aligned}
& R_{1}=\frac{(\Sigma H) \cdot a}{2(c-a)}+\frac{W\left(7 c^{2}-13 c a+a^{2}\right)}{16 c(c-a)} ; . . \\
& R_{2}=\frac{(\Sigma H) \cdot a}{2(c-a)}+\frac{W\left(c^{2}-3 c a-a^{2}\right)}{16 c(c-a)} . . .
\end{aligned}
$$

The values of $V_{1}$ and $V_{2}$ can be found as explained in Art. 28, using either the entire system of loads or the external forces of Fig. $50 \mathrm{~A}$. 
Having determined the above quantities, the beam stresses in the columns are calculated from the loads of Figs. $5 \circ \mathrm{B}$ and C, while the direct stresses in the frame can be found by drawing a stress diagram for the joint loads (Fig. $5 \circ \mathrm{A}$ ).

\section{Case II. Columns Hinged at Base and Loaded Only at} the Joints. This is a special case under Case I, the value of the loading on the columns, other than at the joints ( $W$ in this work), being zero. Substituting $W=0$ in Eqs. (I I), (I2), (I3), (I4), (I5), and (I6) we have

$$
\begin{aligned}
& S_{1}=S_{2}=\frac{(\Sigma H) \cdot c}{2(c-a)} ; \quad \cdot . \cdot \cdot \\
& H_{1}=H_{2}=\frac{\Sigma H}{2} ; \quad \bullet \cdot \bullet \cdot \bullet \\
& R_{1}=R_{2}=\frac{(\Sigma H) \cdot a}{2(c-a)} \cdot \text {. } \cdot \text { ・ }
\end{aligned}
$$

Approximate Solution of Case I. An approximate solution of Case I may be made by substituting for the intermediate column loading ( $W$ in this work) its components at the adjacent joints, $e$ and $d$, or $d$ and $b$ (Fig. 49), and using the formulas of Case II. The error involved will evidently vary with each individual case (see Art. 66).

63. Case III. Columns Fixed at the Base. The Joints of the Frame may be subjected to any System of Loads; the Columns, in addition, being loaded at Other Points than the Joints. As in Case I the intermediate column loading will be taken to be a uniformly distributed wind load $W$.

Assumptions. We assume as before that the truss is comparatively rigid, the points $e, d, e^{\prime}$, and $d^{\prime}$ deflecting equally, while the columns bend as shown by the dotted lines $e d b_{2}$ and $e^{\prime} d^{\prime} b_{2}^{\prime}$ of Fig. 49. 
Separation of Loads. Treating the columns as beams fixed in direction at the base, the loading is separated (see Art. 6r) as shown in Fig. $5 \mathrm{I}$.

Determination of Values of $R_{1}, S_{1}, R_{2}$, and $S_{2}$. It follows from the assumptions just stated that the deflections of the columns

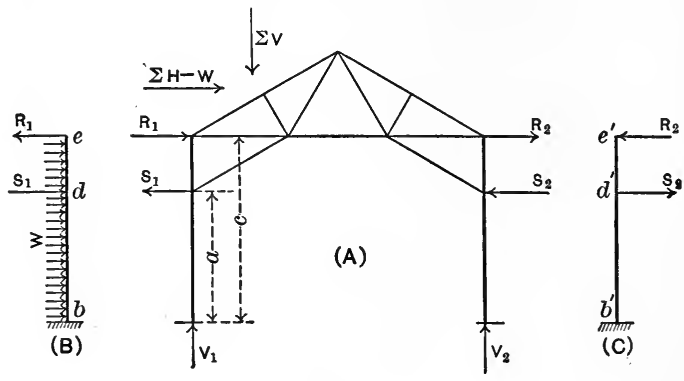

FIG. 5I.

at $e, d, e^{\prime}$, and $d^{\prime}$ are equal. From the ordinary formulas for slope and deflection of a beam fixed at one end we have

$$
\text { (due to } S_{1} \text { ) (due to } W \text { ) (due to } R_{1} \text { ) }
$$

Deflection at $e=\frac{S_{1}}{E I}\left(\frac{a^{3}}{3}+\frac{a^{2}(c-a)}{2}\right)+\frac{W c^{3}}{8 E I}-\frac{R_{1} c^{3}}{3 E I} ;$

$$
\text { (due to } S_{1} \text { ) (due to } W \text { ) }
$$

Deflection at $d=\frac{S_{1} a^{3}}{3 E I}+\frac{W}{24 c E I}\left(6 c^{2} a^{2}-4 c a^{3}+a^{4}\right)-\frac{R_{1}}{E I}\left(\frac{c a^{2}}{2}-\frac{a^{3}}{6}\right)$;

$$
\text { (due to } S_{2} \text { ) (due to } R_{2} \text { ) }
$$

Deflection at $e^{\prime}=\frac{S_{2}}{E I}\left(\frac{a^{3}}{3}+\frac{a^{2}(c-a)}{2}\right)-\frac{R_{2} c^{3}}{3 E I} ; \quad \cdot . \cdot \cdot$

$$
\text { (due to } S_{2} \text { ) (due to } R_{2} \text { ) }
$$

Deflection at $d^{\prime}=\frac{S_{2} a^{3}}{3 E I}-\frac{R_{2}}{E I}\left(\frac{c a^{2}}{2}-\frac{a^{3}}{6}\right) . \quad$. . . . . (4) 
Equating (I) and (3) and reducing, we have

$$
\left(S_{1}-S_{2}\right) \frac{3 a^{2} c-a^{3}}{6}-\left(R_{1}-R_{2}\right) \frac{c^{3}}{3}=-\frac{W c^{3}}{8} . \text {. }
$$

Equating (I) and (2) and reducing, we have

$$
S_{1} a^{2}=\frac{R_{1}}{3}\left(2 c^{2}+2 c a-a^{2}\right)-\frac{W}{\mathrm{I} 2 c}\left(3 c^{3}+3 c^{2} a-3 c a^{2}+a^{3}\right) .
$$

Equating (3) and (4) and reducing, we have

$$
S_{2} a^{2}=\frac{R_{2}}{3}\left(2 c^{2}+2 c a-a^{2}\right) \text {. . . . . . . • • }
$$

From $\Sigma H=0$, Fig. 5I A, we have

$$
\left(S_{1}+S_{2}\right)-\left(R_{1}+R_{2}\right)=\Sigma H-W . . \quad . . .
$$

From equations (5), (6), (7), and (8) we have finally

$$
\begin{aligned}
& R_{1}+R_{2}=\Sigma H \frac{3 a^{2}}{2(c-a)(c+2 a)}+W \frac{3 c^{3}+3 c^{2} a-15 c a^{2}+a^{3}}{8 c(c-a)(c+2 a)} \\
& R_{1}-R_{2}=W \frac{6 c^{3}-12 c^{2} a+6 c a^{2}-a^{3}}{4 c(c-a)(4 c-a)} ; . . . . . . \\
& S_{2}=R_{2} \frac{2 c^{2}+2 c a-a^{2}}{3^{a^{2}}} ; \text {. . . . . . . } \\
& S_{1}=\Sigma H-W+\left(R_{1}+R_{2}\right)-S_{2} \text {. . . . . • }
\end{aligned}
$$

From eqs. (9), (IO), (I I), and (12) the values of $R_{1}, R_{2}, S_{1}$, and $S_{2}$ can be readily determined.

The beam stresses in the columns and the direct stresses in all the members of the frame can now be found as described for Case I.

64. Case IV. Columns Fixed at Base and Loaded Only at the Joints. This is a special case under Case III, the value of the 
loading on the columns, other than at the joints ( $W$ in this work) being zero. Substituting $W=0$ in (9), (I0), (II), and (I2), and reducing, we have

$$
\begin{aligned}
& R_{1}=R_{2}=\Sigma H \frac{3 a^{2}}{4(c-a)(c+2 a)} ; . . . \\
& S_{1}=S_{2}=\Sigma H \frac{2 c^{2}+2 c a-a^{2}}{4(c-a)(c+2 a)} . \quad . . .
\end{aligned}
$$

Approximate Solution. An approximate solution of Case III may be made as described for Case I.

65. Example. Fig. 52 represents an intermediate bent of a steel framed building, the sides of which are corrugated iron attached to the columns. It is required to determine the stresses due to wind pressure on the left-hand side(columns fixed at base).

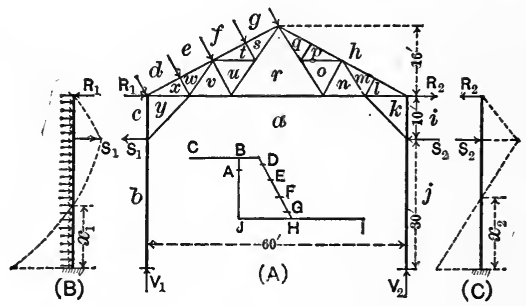

FIG. $5^{2}$.

The loads are separated as explained in Art. 63 into: I. Those causing beam stresses in the columns (Fig. $5^{2} \mathrm{~B}$ and C); 2 . Those causing direct stresses in the frame (Fig. $5^{2} \mathrm{~A}$ ).

Distance between bents

Assumed wind load on a vertical surface Total wind load on column $=W$ Normal wind load on roof (Hutton's formula) = I9 lbs. per sq. $\mathrm{ft}$. 
Total wind load (normal) on roof $=P=10336 \mathrm{lbs}$.

Horizontal component of $P$

Vertical component of $P$

$\Sigma H=19,200+4864$

a

$c$
$=4864 \mathrm{lbs}$.

$\doteq 9 \mathrm{r} 20 \mathrm{lbs}$.

$=24064 \mathrm{lbs}$.

$=3 \mathrm{ot}$.

$=40 \mathrm{ft}$.

Substituting these values in (9), (IO), (II), and (I2), Art. 63, we obtain

$R_{1}=10795$ lbs.; $R_{2}=1107$ I lbs.; $S_{1}=7457$ lbs.; $S_{2}=19273 \mathrm{lbs}$.

Figs. $5^{2} \mathrm{~B}$ and $5^{2} \mathrm{C}$ can now be solved as beams fixed at one end, with the following results:

Windward column (Fig. 52 B):

Maximum shearing fo:ce (at base) $=15862 \mathrm{lbs}$.

Maximum bending moment (at base) $=\mathrm{r} 759$ ro ft.-lbs.

The bending-moment diagram (see Chap. III) is shown by dotted lines.

The point of inflexion (bending moment=0) is distant $x_{1}=$ I4.I $\mathrm{ft}$. from base.

Leeward column (Fig. $\left.5^{2} \mathrm{C}\right)$ :

Maximum shearing force (upper segment) = IIO7I lbs.

Shearing force in lower segment, including base $=8202 \mathrm{lbs}$.

Maximum bending moment (at base) $\quad=\mathrm{I} 35350 \mathrm{ft}$. $-\mathrm{lbs}$.

The bending-moment diagram (see Chap. III) is shown by dotted lines.

The point of inflexion (bending moment $=0$ ) is distant $x_{2}=\mathrm{I} 6.5 \mathrm{ft}$. from base. 
Direct stresses in frame (Fig. $5^{2} \mathrm{~A}$ ):

Taking moments about base of right-hand column, we have $60 V_{1}=\left(S_{1}+S_{2}\right) 30-\left(R_{1}+R_{2}\right) 40+9 \mathrm{I} 20 \cdot 45-4864 \cdot 48 ;$

$\therefore V_{1}=1736 \mathrm{lbs}$.

and $\quad V_{2}=9120-1736=7384 \mathrm{lbs}$.

The values of $V_{1}$ and $V_{2}$ might also be found by using the actual system of loads on the frame (Art. 28).

The direct stresses in the frame can now be found by constructing a stress diagram for the loads of Fig. $5^{2}$ A. Fig. $5^{2} \mathrm{~A}$ is suitably lettered for this purpose and the external force polygon $A B C D E F G H I J A$ is drawn.

The cross-section of the columns being known, the bending stresses can be computed and combined with the direct compression stresses given in the stress diagram (see Art. 57).

66. Approximate Solution of the Example, Art. 65. (See Art. 64.) Resolving the wind load on the column into components at the adjacent joints, we obtain (see Fig. 53) 2400 lbs. at $e_{1}, 9600 \mathrm{lbs}$. at $d_{1}$, and $7200 \mathrm{lbs}$. at $b_{1}$; the last being shown in Fig. $53 \mathrm{~B}$, since its effect is to cause a shearing stress at the base of the column rather than direct stresses in the frame. In this case $\Sigma H=4864+2400+9600=\mathrm{I} 6864$ lbs.

Substituting in (I3) and (I4), Art. 64 we obtain

$$
\begin{aligned}
& R_{1}=R_{2}=I^{2} 3^{8} 3 \mathrm{lbs} . \\
& S_{1}=S_{2}=\mathrm{I} 98 \mathrm{I} 5 \mathrm{lbs} .
\end{aligned}
$$

Separating the loads as before, we obtain the systems of forces shown in Fig. $53 \mathrm{~A}, \mathrm{~B}$, and C. Solving, we obtain the following results: 
Windward column (Fig. 53 B):

Maximum shearing force (at base) $=15632 \mathrm{lbs}$.

Maximum bending moment (at base) $=\mathrm{I} 39 \mathrm{I} 30 \mathrm{ft}$.-lbs.

The poirt of inflexion (bending moment $=0$ ) is $16.5 \mathrm{ft}$. above the base.

Leeward column (Fig. $53 \mathrm{C}$ ):

Maximum shearing force (upper segment) $\quad=11383 \mathrm{lbs}$.

Shearing force in lower segment, including base $=843^{2} \mathrm{lbs}$.

Maximum bending moment (at base) $\quad=$ I $39 \mathrm{I} 30 \mathrm{ft}$. - lbs.

The point of inflexion is $16.5 \mathrm{ft}$. above base.

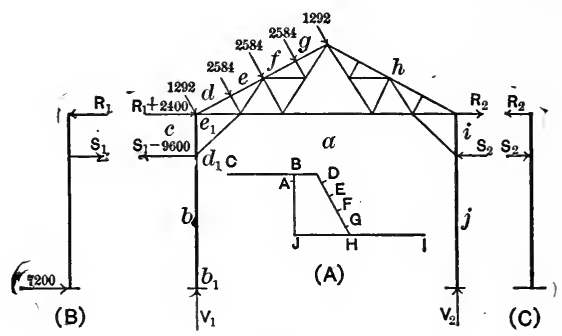

FIG. 53.

Direct Stresses in Frame (Fig. 53 A). Taking moments about base of right-hand column, we have

$60 V_{1}=\left(S_{1}+S_{2}-9600\right) 30-\left(R_{1}+R_{2}+2400\right) 40+9$ I $20 \cdot 45-4864 \cdot 48 ;$

$\therefore V_{1}=$ II 86 lbs.

and $V_{2}=9120-V_{1}=7934 \mathrm{lbs}$.

The polygon of external forces is given in Fig. $53 \mathrm{~A}$.

A comparison of the stress diagrams (not drawn) for Figs. $52 \mathrm{~A}$ and $53 \mathrm{~A}$, together with the relative values of maximum shearing 
force and bending moment in Arts. $6_{5}$ and 66, will serve to indicate the difference in the results obtained, in this particular example, by the two methods of solution.

When such frames are hinged at the bases of the columns the stresses in general are larger than when fixed at the base.

67. Problems. I. Solve the example of Art. 65, assuming the columns hinged at the base. Compare the results with those of Art. 65

2. Solve Problem I by the approximate method, and compare results with those of Art. 66. Also compare the results with those of Problem I.

3. Determine the maximum bending moment, maximum shearing force, and points of inflexion for the columns of Fig. 54,

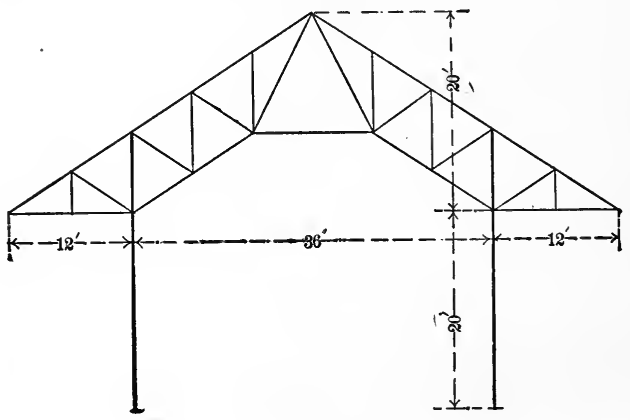

FIG. 54.

due to a vertical load of 20000 lbs. uniformly distributed over the roof surface, together with a normal wind load of I2500 lbs. on the inclined roof surface. Also determine the direct stresses in all the members of the frame. Assume the columns to be hinged at the base. 
4. Solve Problem 3, assuming the columns to be fixed at the base.

68. Sway-bracing. Illustration. The frame (Fig. 55) is made up of a number of transverse frames or bents, braced so as to resist distortion of any kind. Each bent is composed of a

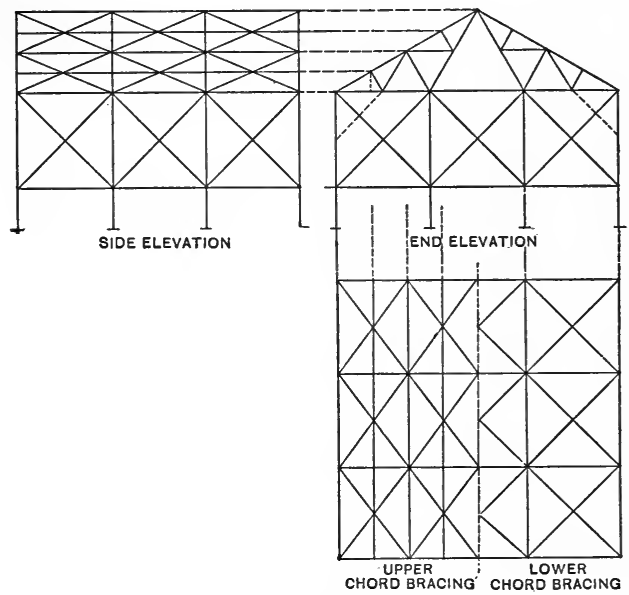

FIG. 55.

truss supported on columns, the truss and columns being braced together as shown in the end elevation. In the intermediate bents knee-braces, shown by the dotted lines, are commonly used; while at the ends two intermediate columns are shown, the bracing for these consisting of diagonal tension-rods and horizontal struts. The bents are braced together by (I) diagonal tension-rods in the plane of the upper chords, the purlins serving as struts; 2) diagonal tension-rods in the plane of the lower 
chords; (3) diagonal tension-rods in the sides of the building between the columns.

Such a system of bracing as described aims to prevent distortion of the individual bents, and to keep the various bents vertical and in line, under the action of any horizontal force such as wind on the side or end of the building, the pull of belts, thrust of jib-cranes, etc. The particular system of bracing em-
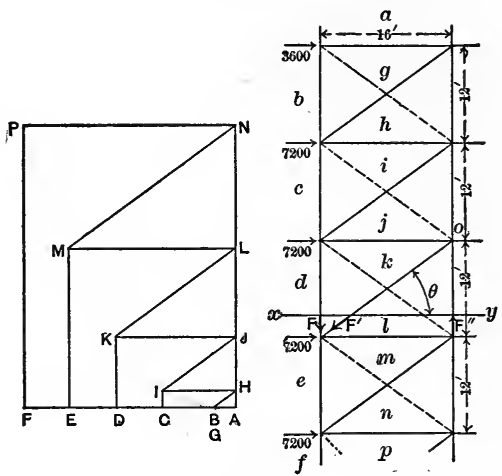

FIG. 56.

ployed will vary with the circumstances. ${ }^{1}$ The stresses in such bracing can usually be best determined by algebraic methods.

The following example indicates a suitable solution for a simple case, the system of bracing shown being, however, generally inconvenient to employ for such structures.

69. Example. The vertical lines (Fig. 56) represent two adjacent tiers of columns in a building of skeleton construction. The horizontal lines are floor girders or special members located

\footnotetext{
${ }^{1}$ See Johnson's “Theory and Practice of Modern Framed Structures"; also Freitag's " Architectural Engineering."
} 
in the floor system; these, with the diagonal tension-rods, forming a system of wind bracing for a width of side wall of $20 \mathrm{ft}$. It is required to find the stresses resulting from a wind pressure of $30 \mathrm{lbs}$. per sq. $\mathrm{ft}$. on the side of the building.

The wind pressure on each panel is $30 \times 12 \times 20=7200 \mathrm{lbs}$. The resulting pressures at the joints are given. Since the diagonals are tension members, those drawn in full lines will be stressed for wind on the side shown (see $\$ 4$ ). The stresses in the members cut by any section, $x y$, may be found algebraically as follows:

Dealing with the forces acting on the portion of the frame above the section, we have (I) $\Sigma H=0$.

$$
\begin{gathered}
\therefore 3600+7200+7200=F^{\prime} \cos \theta=.8 F^{\prime} . \\
\therefore F^{\prime}=22500 \text { lbs. (tension). }
\end{gathered}
$$

(2) Taking moments about $O$,

$$
3600 \cdot 24+7200 \cdot 12=\mathrm{I} 6 F . \quad \therefore F=10800 \mathrm{lbs} \text {. (tension). }
$$

(3) From $\Sigma M=0$ or $\Sigma V=0$ we find the compression stress in the leeward column to be 24300 lbs.

To find the stress in $j k$ we have, dealing with the joint $O$, $\Sigma H=0$.

$\therefore$ Compression stress in $j k=F^{\prime} \cos \theta=\mathrm{I} 8000 \mathrm{lbs}$.

The stress diagram for this example is given in Fig. $5^{6 .}{ }^{1}$

\footnotetext{
${ }^{1}$ See Freitag's “ Architectural Engineering” for discussion of sway-bracing in steel framed buildings of skeleton construction.
} 


\section{CHAPTER III.}

BEAMS.

\section{$\S \mathrm{I}$. Shearing Force and Bending Moment.}

70. Definitions. The shearing force $(S)$ at any cross-section of a beam is the measure of the tendency of the external forces to cause the portion of the beam lying on one side of the section to slide over the remaining portion, this tendency being opposed by the resistance to transverse shearing of the material. The value of $S$ is found by taking the algebraic sum of the external forces lying on either side of the section.

The bending moment $(M)$ at any cross-section of a beam is the measure of the tendency of the external forces to cause the portion of the beam lying on one side of the section to rotate about the section, this tendency being opposed by the resistance to longitudinal tension and compression of the material. The value of $M$ is found by taking the algebraic sum of the moments of the external forces lying on either side of the section, about the neutral axis of the section.

7I. Graphical Representation of $S$ and $M$. The beam (Fig. 57) is supported at the ends and loaded with three concentrated loads. These loads, $A B, B C$, and $C D$, are plotted to scale, and the supporting forces, $D E$ and $E A$, are determined by constructing the funicular polygon (Fig. $57 \mathrm{~A}$ ).

In the $S$ diagram (Fig. 57 B) any ordinate from $X X^{\prime}$, e.g., $n^{\prime} m^{\prime}$, represents the value of $S$ at the corresponding section of the beam. 
These ordinates are taken from the external force polygon and plotted as indicated. Thus at any section in space $b, S=E A-$ $A B=E B$, etc. The following points should be noted: r. At

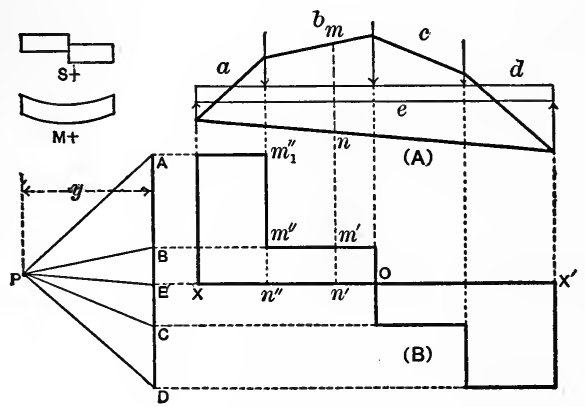

Fig. 57.

any concentrated load, e.g., $a b$, there are two ordinates, $n^{\prime \prime} m_{1}^{\prime \prime}$ and $n^{\prime \prime} m^{\prime \prime}$. These represent the values of $S$ at sections immediately to the left and right of the load, respectively, and differ in value by the amount of the load. 2. $S$ has opposite signs on the two sides of the load $b c$, hence passes through the value zero at this section. 3. $S$ is constant for any unloaded portion of a beam, and varies uniformly in case of a uniformly distributed load; the corresponding portions of the $S$ diagram consisting of horizontal and inclined straight lines respectively (see Figs. 57 to 62 ).

$M$ Diagram. It has been shown (Art. 20) that in case of a beam, any intercept, e.g., $m n$, of a funicular polygon multiplied by the pole distance, $y$ (proper attention being given to the scales of the force and funicular polygons), is the value of $M$ at the section in question. Since the pole distance is constant, the intercepts are proportional to the values of $M$. A funicular polygon for the external forces acting on the beam therefore con- 
stitutes the $M$ diagram. In constructing this diagram the pole distance should be taken to represent a convenient number of units, e.g., Iooo lbs. The method for determining the strings between which any intercept is measured is as follows: The value of $M$ at any section, e.g., $m n$, is, by definition, equal to the algebraic sum of the moments of the forces ( $E A$ and $A B$ ) lying to one side of the section. The strings in question are the ones between which these forces lie, i.e., $e$ and $b$ in this instance. It should be noted that in case of any system of concentrated loads the $M$ diagram is made up of straight lines, while the portion of the diagram corresponding to a uniformly distributed load is a parabola which can be constructed as explained in Art. I3.

Algebraic Signs of $S$ and $M$. The following arbitrary rules are in vogue: $S$ is positive if the tendency is for the left-hand portion of the beam to slide upwards relatively to the right-hand portion and conversely. $M$ is positive if the tendency is for the beam to bend convex downward and conversely (see Fig. 57). In the $S$ diagrams positive ordinates will be plotted upwards. In the $M$ diagrams positive intercepts will lie above the closing strings of the funicular polygon provided that the pole be taken on the left side of the force polygon (see Figs. 57 to 62 ).

72. Relation between $S$ and $M$ Diagrams. The algebraic condition for maxima and minima applied to bending moments is $\frac{d M}{d x}=0$. It is proved in "Strength of Materials" that $\frac{d M}{d x}=S$. Hence, in general, the sections of a beam at which the $M$ diagram intercepts are greatest (or least) correspond with those where the $S$ diagram cuts the $X$ axis (see Figs. 57, 59, 61, 62). This relation between the two diagrams does not in certain cases (see Fig. 58) hold true, the reason being that the numerically greatest (or least) value of an ordinate, occurring within a limited 
space, is not necessarily a maximum or minimum value in the mathematical sense.

\section{Relation between Moment Diagram and Elastic Curve.} $M$ is assumed to be positive where the beam bends convex downward and conversely, so that the general shape of the elastic curve may be determined by observing the algebraic sign of $M$ in the various parts of the beam as indicated in the $M$ diagram. The sections at which $M$ changes sign and hence equals zero evidently correspond to points of inflexion of the elastic curve. The general form of these curves, determ ned in this manner, for Examples 4 and 5 (see Art. 74), are represented in Figs. 6r and 62 respectively, the points of inflexion being marked with circles.

The following examples will serve to indicate suitable methods for constructing the $S$ and $M$ diagrams.

74. Examples. I. The cantilever, Fig. 58, is loaded with a uniformly distributed load (resultant $A B$ ) and a concentrated load $B C$ as shown. The $S$ diagram (Fig. $5^{8} \mathrm{~B}$ ) needs no ex-
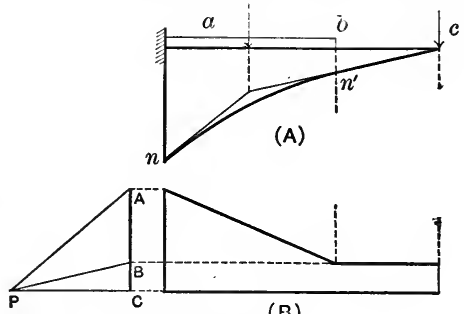

(B)

Fig. $5^{8 .}$

planation. The $M$ diagram (Fig. $5^{8} \mathrm{~A}$ ) is constructed as follows: Substitute for the distributed load its resultant (represented by the dotted arrow), and construct the funicular polygon, beginning at the free end. By taking the pole on a horizontal line through 
$C$, the string $c$ may be taken to coincide with the beam; the strings $b$ and $a$ are then drawn. The true diagram for the distributed load is a parabola tangent to the strings $a$ and $b$ at the points $n$ and $n^{\prime}$, corresponding to the ends of the load. The parabola is constructed as explained in Art. 13.

Example 2. The beam (Fig. 59) is supported at the ends and loaded with the uniformly distributed load shown. The supporting forces, $B C$ and $C A$, are determined by constructing

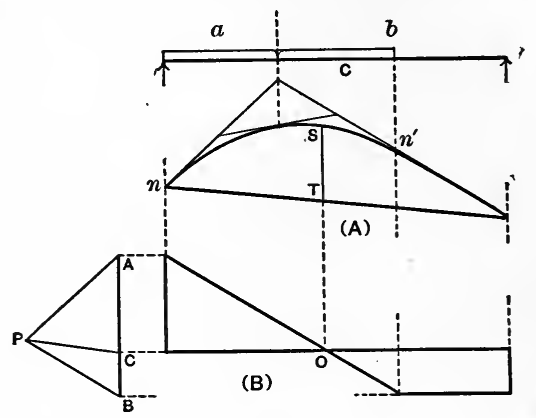

FIG. 59 .

a funicular polygon for the resultant load $A B$. The actual diagram for the distributed load is a parabola tangent to the strings $a$ and $b$ at $n$ and $n^{\prime}$ respectively. The true funicular polygon thus formed, shown by heavy lines in Fig. $59 \mathrm{~A}$, constitutes the $M$ diagram. The $S$ diagram, Fig. $59 \mathrm{~B}$, needs no explanation.

From the $S$ diagram it is seen that $S$ is zero at the point $O$, the maximum intercept $S T$, and hence maximum $M$ occurring at this section of the beam.

Example 3. If, in case of a distributed load, the value of $M$ at some designated section of the beam is wanted, it can be found, without constructing the funicular curve, by making such section 
a point of division of the load (Art. 12). Fig. 60 represents a beam supported at the ends and loaded uniformly. It is required to find the value of $M$ at the middle of the beam. Making

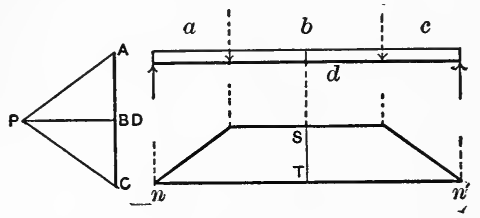

Fig. 60.

this section a point of division of the load, we substitute for each half-load the resultants $A B$ and $B C$ and construct the funicular polygon shown. The ordinate $S T$ multiplied by the pole distance gives the value of $M$ at the section in question. In the true $M$ diagram a parabola tangent to the strings $a, b$, and $c$ at, $n, S$, and $n^{\prime}$ respectively, would take the place of these strings.

ExAMPLE 4. Fig. 6I represents a beam overhanging its right-hand support and loaded with a uniformly distributed load over its entire length together with two concentrated loads $B C$ and $D E$. In place of the two segments of the distributed load we substitute their resultants $A B$ and $C D$ and construct the funicular polygon, Fig. 6I A. The closing string $f$ and corresponding ray determine the supporting forces $E F$ and $F A$. A parabola tangent to the strings $a$ and $b$ at $n$ and $S$ respectively is the true diagram for the left-hand segment of the distributed load, while another parabola tangent to the strings $c$ and $d$ at $S$ and $n^{\prime}$ respectively is the true diagram for the right-hand segment. The $M$ diagram is drawn in heavy lines.

The $S$ diagram is constructed as follows: The value of $S$ at the left-hand support is $F A$ (reaction of support). This distance is taken from the external force polygon and plotted in 
the $S$ diagram (lettered $F^{\prime} A^{\prime}$ ). On the left- and right-hand sides of the load $B C$ the values of $S$ are $F A-A B=F B$ and $F A-A B-B C=F C$. These values are plotted and lettered $F^{\prime} B^{\prime}$ and $F^{\prime} C^{\prime}$ respectively. On the left- and right-hand sides of the supporting force $E F$, the values of $S$ are $F A-A B-B C-C X$ $=F X$ and $F A-A B-B C-C X+E F=E X$ (lettered $F^{\prime} X^{\prime \prime}$ and $E^{\prime} X^{\prime}$ respectively). The load $C X$ is the portion of $C D$ lying
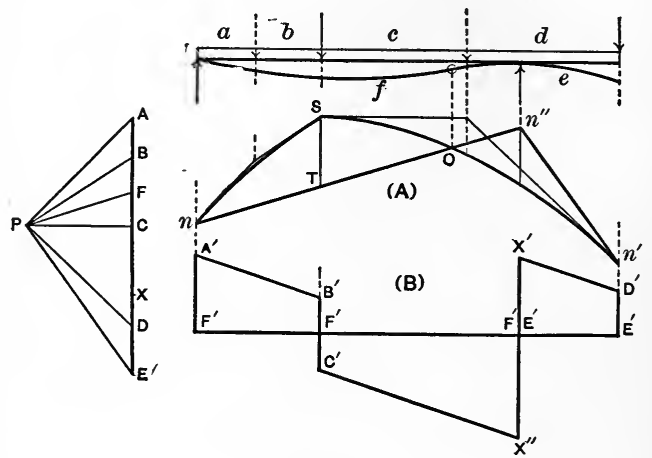

Fig. 6r.

to the left of the right-hand support. Finally, the value of $S$ at the right end of the beam is $D E . S$ varies uniformly between these sections (see Art. $7 \mathrm{I}$ ).

Max. $S\left(F^{\prime} X^{\prime \prime}\right)$ occurs immediately to the left of the righthand support.

The maximum values of $M(S=0)$ occur at the load $B C$ and at the right-hand support, the numerically greater value being at the load $B C$ as is seen by inspection of the $M$ diagram. From the $M$ diagram it is seen that $M=0$ at the point $O$, being positive to the left and negative to the right of this section.

The general form of the elastic curve is indicated in the upper- 
most diagram, its point of inflexion, marked by a circle, being at the section $O$.

Example 5. Fig. 62 represents a beam made up of two segments hinged together as indicated. It is supported at three equidistant points and loaded uniformly, the resultant loads on
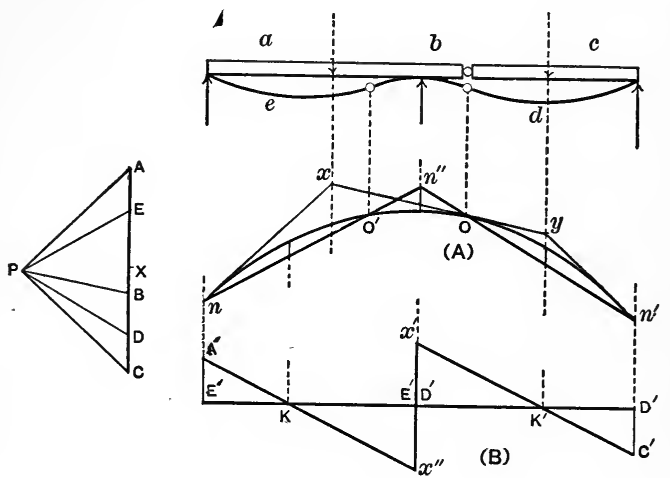

FIG. 62.

the two segments being $A B$ and $B C$. A funicular polygon for these resultant loads is constructed as follows: Since there can be no bending moment at the hinge, the intercept between the strings $b$ and $d$ must be zero at that point. Hence the construction is begun by drawing the string $b(=x y)$ in any convenient direction and the corresponding ray; the pole $P$ being taken on this ray: The strings $a$ and $c$ are next drawn. The string $d$ must now pass through the points $n^{\prime}$ and $O$, intersecting the middle support at $n^{\prime \prime}$. This determines the closing string $e$ $\left(n n^{\prime \prime}\right)$. Rays parallel to the strings $d$ and $e$ determine the supporting forces $C D, D E$, and $E A$.

The true diagram for the distributed load is a parabola 
tangent to the strings $a, b$, and $c$ at the points $n, O$, and $n^{\prime}$ respectively. The $M$ diagram is drawn in heavy lines.

The $S$ diagram, Fig. $62 \mathrm{~B}$, is constructed as described for Example 4, $A X=X C=\frac{1}{2} A C$ being the resultant loads on the two sides of the middle support. Max. $S$ occurs on both sides of the middle support.

Max. $M(S=0)$ occurs at $K, K^{\prime}$, and the middle of the beam, the greatest value being at the middle of the beam as shown by inspection of the $M$ diagram. $M$ is zero at $O$ and $O^{\prime}$ (Fig. 62 A), these points being consequently the points of inflexion of the elastic curve. This curve is drawn, one point of inflexion being at the hinge.

75. Problems. I. Given a beam, i6 ft. span, supported at both ends and loaded with a uniformly distributed load of rooo lbs. per foot, extending over the left half-span. Construct the $S$ and $M$ diagrams and determine the values of max. $S$ and max. $M$ graphically. Check the graphical work by determining these values algebraically.

Problem 2. Given a beam supported at two points $\mathrm{I} 6 \mathrm{ft}$. apart and overhanging the right-hand support 4 feet. It is loaded with a uniformly distributed load of rooo lbs. per ft. covering the left half-span ( $8 \mathrm{ft}$.), also with a concentrated load of $6000 \mathrm{lbs}$. at the extreme right end of the beam. Construct the $S$ and $M$ diagrams and determine the values of max. $S$ and $\max . M$ both graphically and algebraically. Also locate the point of inflexion of the elastic curve both graphically and algebraically.

Problem 3. Construct the $S$ and $M$ diagrams for the columns of Fig. $5^{2}$ and determine the values of $\max . S$ and max. $M$ for each graphically. 
§2. Deflection of Beams.

II - 76. Graphical Determination of Elastic Curve. In simple cases the usual formulas furnish the best method for determining the deflection of a beam. In the case of beams of non-uniform section, or loaded in a complex manner, a graphical solution can be employed advantageously where extreme accuracy is not. required.

Example. The beam (Fig. 63), of uniform section, is supported at the ends and loaded with two concentrated loads as shown. It is required to construct the elastic curve.

Salution. Construct the funicular polygon (Fig. 63 A) for the given loads. The force diagram is Fig. $63 \mathrm{~A}^{\prime}$. Treat the surface

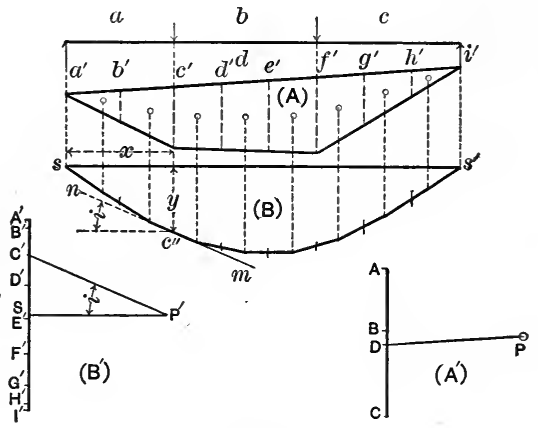

FIG. 63 .

of this polygon as if it represented a distributed load for the same beam, and construct a second funicular polygon. For this purpose the surface (Fig. $63 \mathrm{~A}$ ) is subdivided by ordinates, and each division is concentrated at its centre of gravity, marked by a circle. The areas of these surfaces are plotted to scale in the force diagram (Fig. $63 \mathrm{~B}^{\prime}$ ), and the corresponding funicular poly- 
gon (Fig. $63 \mathrm{~B}$ ) is drawn. The exact diagram will be a curve inscribed in this polygon, its tangent points corresponding to the points of division of Fig. $63 \mathrm{~A}$. This curve is the elastic curve of the beam, the deflection at any point being represented to scale by the length of the intercept between the curve and the line $S S^{\prime}$, this line being drawn so as to satisfy the condition that the deflection at each support is zero.

\section{Proof:}

Let $O=$ any ordinate of Fig. $63 \mathrm{~A}$ (inches, full size).

“ $D=$ pole distance in Fig. $63 \mathrm{~A}^{\prime}$ (lbs.).

“ $D^{\prime}=$ pole distance in Fig. $6_{3} \mathrm{~B}^{\prime}$ (sq. inches, full size).

" $M=$ bending moment at any section of the beam (inch-lbs.).

“ $E=$ modulus of elasticity of material (lbs. per sq. inch).

" $I=$ moment of inertia of beam section (inches).

Take the origin of coordinates at $S, X$ axis, horizontal, and $Y$ axis, vertical. (For convenience, the pole $P^{\prime}$ is here taken so that $S S^{\prime}$ is horizontal.) Let $m n$ be tangent to the elastic curve at any point $C^{\prime \prime}$ whose coordinates are $x, y$. The rays $P^{\prime} S$ and $P^{\prime} C^{\prime}$ are parallel respectively to $S S^{\prime}$ and $m n$. Hence

$$
\tan i=\frac{d y}{d x}=\frac{S C^{\prime}}{P^{\prime} S}=\frac{S A^{\prime}-A^{\prime} C^{\prime}}{P^{\prime} S=D^{\prime}} . . . . .
$$

In Eq. I, $S A^{\prime}$ is constant and $A^{\prime} C^{\prime}$ is equal to the area in Fig. $63 \mathrm{~A}$, lying to the left of the point being considered; i.e.,

$$
A^{\prime} C^{\prime}=\int_{0}^{x} O d x
$$

Substituting in Eq. I,

$$
\frac{d y}{d x}=\frac{S A^{\prime}-\int_{0}^{x} O d x}{D^{\prime}} .
$$


Differentiating and dividing by $d x$, we have, neglecting signs,

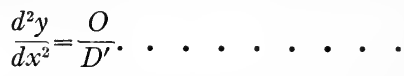

The general (approximate) equation of the elastic curve is

$$
\frac{d^{2} y}{d x^{2}}=\frac{M}{E I} \text {. . . . . . . . . }
$$

Hence the curve of Fig. $63 \mathrm{~B}$ will be the true elastic curve provided that the second members of Eqs. 2 and 3 are equal, i.e.,

$$
\frac{O}{D^{\prime}}=\frac{M}{E I}=\frac{O \cdot D}{E I} ; \therefore D D^{\prime}=E I . \quad . \quad . .
$$

If the pole distances are taken at random, the relative lengths of the ordinates, Fig. $6_{3} \mathrm{~B}$, will represent the relative deflections at the various points of the beam. If $D$ and $D^{\prime}$ are taken so as to satisfy Eq. 4, the scales of abscissas and ordinates will be the same. It is desirable to magnify the ordinates so as to determine the deflections more accurately than otherwise. Thus if the scale of abscissas is I: $a$ and we wish to make the ordinates in the drawing $n$ times as great as the actual deflections, we must make the scale of ordinates $n \times a$ as great as the scale of abscissas in Fig. $6_{3} \mathrm{~B}$. For this purpose $D D^{\prime}$ must be reduced in the same ratio (see Art. 20) and Eq. 4 then becomes

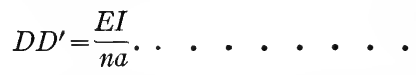

By means of Eq. 5 suitable values of $D$ and $D^{\prime}$ can be selected such that the ordinates of Fig. $6_{3} \mathrm{~B}$ will represent the deflections of the beam magnified $n$ times.

It is not necessary, in determining deflections, for $S S^{\prime}$ (Fig. $63 \mathrm{~B})$ to be horizontal, since the ordinates remain the same so long as the pole distance $D^{\prime}$ is not altered. 
If the bending moment changes sign, the areas (Fig. 63 A) corresponding to negative moments must be plotted in the force polygon (Fig. $6{ }_{3} \mathrm{~B}^{\prime}$ ) in the opposite direction from those corresponding to positive moments (see Example 2, Art. 77).

If the beam is of non-uniform section, Eq. 5 shows that $D D^{\prime}$ must vary in the same ratio as $I$. This is accomplished by varying the pole distance $D^{\prime}$ (see Example I, Art. 77).

To determine the deflection at any given section of the beam make one division line of the moment diagram (Fig. 63 A) correspond to such section (compare with Example 3, Art. 74). Otherwise the moment diagram may be subdivided in any manner, except in case of beams of non-uniform section, for which see Example I, Art. 77 .

77. Examples. I. Fig. I, Plate II, is a cantilever, Io ft. span, loaded at two points. $I$ has three different values, as given in the diagram. $E=30000000$ lbs. per. sq. in. It is required to construct the elastic curve and determine the deflection at the free end of the beam.

Scale $^{1}$ of space diagram, I: 20. $\therefore a=20$. Scale of force diagram (Fig. $\mathrm{I} \mathrm{A}^{\prime}$ ), $4000 \mathrm{lbs}=\mathrm{I}^{\prime \prime}$. The pole distance $P A$ is taken to be $4^{\prime \prime}$. $\therefore D=4 \times 4000=\mathrm{I} 6000$. The construction of the moment diagram (Fig. I A) needs no explanation. Its surface is divided as shown and the centres of gravity of the divisions are indicated by circles. The areas of these divisions are plotted in Fig. $\mathrm{I} \mathrm{B}^{\prime}$ to the scale, rooo sq. in. (full size) $=\mathrm{I}^{\prime \prime}$. The full-size area is obtained by multiplying the diagram area by $a^{2}=(20)^{2}=$ 400. The deflections are to be magnified five times, i.e., $n=5$.

Substituting the preceding values in the formula $D D^{\prime}=\frac{E I}{n a}$,

1 The scales referred to here are those of the original drawings, which have been reduced about one-half in the plate. 
we have $16000 D^{\prime}=\frac{30000000 \cdot 200}{5 \cdot 20} . \therefore D^{\prime}=3750=3 \cdot 75^{\prime \prime}$. The pole $P^{\prime}$ is taken with a pole distance of $3 \cdot 75^{\prime \prime}$, and the elastic curve (Fig. I B) is then constructed as follows: The strings corresponding to $P^{\prime} G^{\prime}, P^{\prime} F^{\prime}, P^{\prime} E^{\prime}$ are drawn in order. At the section $e^{\prime}$ the value of $I$ changes to ${ }^{5}$, and the pole distance must be reduced in the same ratio, the new pole $P^{\prime \prime}$ lying on $P^{\prime} E^{\prime}$. The construction is continued in a similar manner, the pole for the portion of the beam where $I=$ roo being $P^{\prime \prime \prime}$. A curve tangent to these strings at the points corresponding to the points of division of Fig. I A is the elastic curve. The ordinate $m n$, divided by 5 , determines the deflection of the beam at the free end to be $496^{\prime \prime}$. The computed deflection is $.493^{\prime \prime}$.

The following points concerning Fig. I B should be noted: I. The vertical scale is $5 \cdot 20=$ Ioo times as great as the horizontal scale. 2. In order to determine the true deflection at the free end, it would have been sufficient to have divided Fig. I A only at the two sections where $I$ changes in value.

If the value of $I$ varied continuously, the divisions of the beam and moment diagram (corresponding to $c^{\prime}$ and $e^{\prime}$, where $I$ changes) should be made sufficiently numerous to insure a close approximation to the true result, the mean value of $I$ for each portion of the beam being employed in the construction of the elastic curve. Otherwise the solution for such a case is similar to that just explained.

Example 2. The beam (Fig. 2, Plate II) is supported at two points $a$ and $h, \mathrm{I}_{4} \mathrm{ft}$. apart, and overhangs $6 \mathrm{ft}$. at the right end. It is loaded with a distributed load of $300 \mathrm{lbs}$. per foot. Cross-section $=6^{\prime \prime} \times \mathrm{I} 2^{\prime \prime} . \quad I=864 . \quad E=\mathrm{I} 200000$. Scale of space diagram, I : 40 .

The load is divided into ten equal lengths, each division being concentrated at its middle point. Scale of force diagram (Fig. $2 \mathrm{~A}^{\prime}$ ), 
2000 lbs. $=\mathrm{I}^{\prime \prime}$. Pole distance $=\mathrm{I}^{\prime \prime}{ }^{\prime \prime}$; i.e., $D=3000$. The surface of the moment diagram ${ }^{1}$ (Fig. 2 A) is divided as shown and the areas plotted in Fig. $2 \mathrm{~B}^{\prime}$ to the scale, 500 sq. in. $=\mathrm{I}^{\prime \prime}$, the positive and negative areas being laid off in opposite directions. $n=5$.

Substituting in the formula $D D^{\prime}=\frac{E I}{n a}$, we have

$$
3000 D^{\prime}=\frac{\mathrm{I} 200000 \cdot 864}{5 \cdot 40} ; \quad \therefore D^{\prime}=\mathrm{I} 728 .
$$

Hence the pole distance (Fig. $2 \mathrm{~B}^{\prime}$ ) is $\frac{1728}{50}=3 \cdot 456^{\prime \prime}$. Fig. $2 \mathrm{~B}$ is the elastic curve. The measured ordinates at $7 \mathrm{ft}$. and $20 \mathrm{ft}$. from the left end, divided by 5 , determine the deflections at these points to be -.I44" and $+.012^{\prime \prime}$ respectively.

The true shape of the elastic curve here would be obtained by plotting its ordinates from a horizontal line, reducing their scale $40 \cdot 5=200$ times.

78. Problem. Given a beam i6 ft. span, supported at both - ends and loaded in the middle with $4000 \mathrm{lbs} ., E=30000000$. In the middle half of the span the cross-section is uniform, the value of $I$ being 300 . Outside the middle half the value of $I$ diminishes at a uniform rate to each support where $I=100$. It is required to determine the deflection at the middle of the span.

(Note. In constructing the elastic curve, divide the middle portion of the span into two 4 -ft. segments and each end portion into four I-ft. segments, using the average value of $I$ in each.)

79. Centre of Gravity. The following constructions for centre of gravity will be found useful in connection with the work of this and the following chapter.

I. Centre of Gravity of Any Quadrilateral Area. Let $A B C D$ (Fig. 64) be the quadrilateral in question. Draw the two diago-

\footnotetext{
${ }^{1}$ This diagram might better have been constructed by the method of Art. 74 .
} 
nals. Bisect one of these, as $A C$, at $E$. Also lay off $D K^{\prime}=B K$ and draw $E K^{\prime}$. Trisect $E K^{\prime}$ at $G$. $G$ is the centre of gravity of the area.

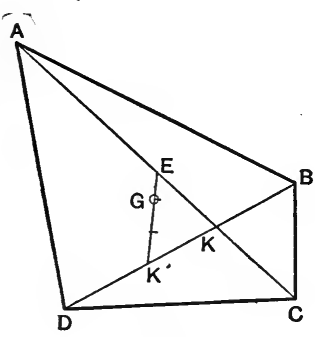

Fig. 64.

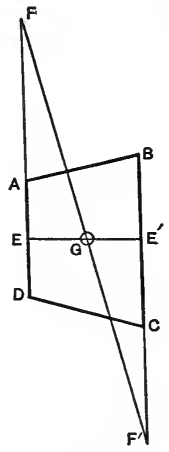

FIG. 65 .

2. Centre of Gravity of a Trapezoid. The following special construction is useful: Let $A B C D$ (Fig. 65) be the trapezoid. Bisect each of the parallel sides and draw the medial line $E E^{\prime}$. Extend the two parallel sides in opposite directions, laying off $A F=B C$ and $C F^{\prime}=A D$. Draw $F F^{\prime}$, intersecting $E E^{\prime}$ at $G$. $G$ is the centre of gravity desired. 


\section{CHAPTER IV.}

\section{MASONRY ARCHES, ABUTMENTS, ETC.}

\section{$\S$ r. General Conditions of Stability.}

8o. Nature of the Forces involved. Let $P R M N$ (Fig. 66) be a block of masonry acted upon by a force $A B$. In addition to this force, the weight of the block must be taken into account. This weight is represented by $B C$, its line of action $b c$ being drawn through the centre of gravity of the block. The resultant of these two forces is $A C$, its line of action passing through the

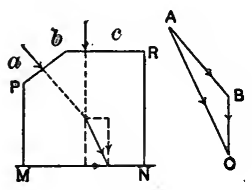

FIG. 66.

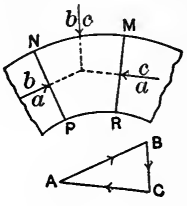

FIG. 67 .

intersection of $a b$ and $b c . A C$ is, therefore, the resultant pressure exerted by the block upon the plane $M N$. This plane may be taken to be a joint of the masonry or its base. Also, the forces which hold the block in equilibrium are $A B, B C$, and the reaction of the plane $M N$, this last being a force equal and opposite to $A C$. Moreover, $A C$ represents the resultant stress on the plane $M N$.

Again, let $P R M N$ (Fig. 67) be an arch-stone, $B C$ being the 
load supported by this stone including its own weight. The line of action $b c$ of this load passes through its centre of gravity. $B C$ is balanced by the forces $A B$ and $C A$ exerted upon $P R M N$ by the adjacent arch-stones. These three forces must, therefore, form a triangle as shown, and their lines of action must intersect at the same point.

8r. Resistance of a Masonry Joint. The conditions of stability for the block of Fig. 66 as far as the joint $M N$ is concerned are evidently the following: (I) the block must not overturn about an edge, as $N$; (2) it must not slide over the joint; (3) the material of the stone and mortar must not crush. These three conditions will be discussed in turn.

82. Resistance to Overturning. In this connection, the tensile strength of the mortar joint is commonly neglected. Then the block (Fig. 66) would evidently overturn, if the line of action of the resultant force $A C$ pierced the plane $M N$ outside of the surface of the joint. The moment of $A C$ about $N$ as moment axis is the measure of the resistance to overturning about this edge, i.e., in order to overturn the block it would be necessary to apply a force whose moment about $N$ was equal to that of $A C$, but having the opposite sign.

83. Resistance to Sliding. Let the resultant force $A C$ (Fig. 66) be resolved into components parallel and perpendicular to the joint, as indicated. The normal component represents the direct pressure on the joint, while the parallel component tends to slide the block over the joint, and must be resisted by the sliding friction at the joint, the adhesion between the stone and mortar being neglected.

Coefficient of Friction. Let $P$ (Fig. 68) be the resultant 
pressure of the block on the plane $A B$, and $\phi$ the minimum

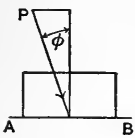

Fig. 68. angle of inclination with the normal at which sliding will occur. This angle $\phi$ is called the angle of repose, and $\tan \phi$, or ratio of tangential to normal component of the force, is called the coefficient of friction. In the case of masonry joints, the value of the coefficient of friction is taken to be from .4 to .5 . In order, then, for sliding not to occur, the resultant pressure at any joint must make with the normal an angle less than $\tan ^{-1} \cdot 4$.

84. Resistance to Crushing. The normal component of $A C$ (Fig. 66) represents the resultant compression stress at the joint $M N$. This stress is assumed to be uniformly varying. The three cases whch may occur are represented in Fig. 69. In Fig. $69 \mathrm{~A}$ the stress is distributed over the whole surface of the joint, the limiting case being Fig. $69 \mathrm{~B}$, where the intensity of stress is zero at one edge $M$. In Fig. ${ }_{9} \mathrm{C}$ the pressure is distributed over the portion $X N$ of the joint. If the joint were capable of resisting tension, this last would be the case where the stress is partly tension and partly compression, but, assuming the joint
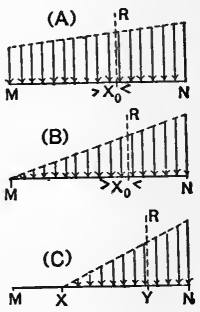

Fig. 69. incapable of resisting tension, the portion $M X$ is without stress and tends to open.

Case of Rectangular Joint. In Fig. $69 \mathrm{~A}$ the maximum intensity of compression (at $N$ ) is given by the formula $f=\frac{R}{A}+\frac{M}{I} y$ (Art. 54), in which $R=$ resultant normal pressure on joint; $A=$ area of surface of joint; $M=$ moment of $R$ about centre of gravity of this surface $=R \cdot X_{0} ; I=$ moment of inertia of surface of joint 
about its centre of gravity, and $y=\frac{1}{2} d \quad(d=M N=d e p t h$ of joint).

In Fig. $69 \mathrm{~B}$ the intensity of compression at $M$ is zero, hence $\frac{R}{A}=\frac{M}{I} y=\frac{6 R X_{0}}{A d} . \therefore X_{0}=\frac{1}{6} d$, or $R$ acts at $\frac{1}{3} M N$ from $N$. Hence: In order for the pressure on a rectangular joint to be distributed over its entire surface, the resultant must act within the middle third of the depth of the joint.

The maximum intensity of stress (at $N$ ) in Fig. $69 \mathrm{~B}$ is evidently double the average stress, i.e., $\frac{2 R}{A}$. Similarly, in Fig. $69 \mathrm{C}$, the maximum stress is double the average stress on the surface $X N=3 Y N$.

Case of Circular Joint. To determine the limits within which $R$ must act in order for the pressure to be distributed over the whole surface of the joint, we have as before $\frac{R}{A}=\frac{M}{I} y=\frac{4 R X_{0}}{A r}$. $\therefore X_{0}={ }_{4}^{1} r$ ( $r=$ radius of joint surface). Hence in this case the resultant must act within one-fourth of the radius from the centre of the joint.

For other forms of joint, the limits within which $R$ must act in order for the pressure to be distributed over the entire area, and the value of the maximum intensity of stress when the pressure is so distributed, are determined in the same manner as indicated above for rectangular and circular surfaces.

\section{Conditions to be Satisfied by Masonry Arches, Abutments,} etc. The following conditions respecting the joints should, in general, be fulfilled:

I. The limits within which the resultant pressure acts should be such that the pressure will be distributed over the entire surface of each joint. 
2. The direction of the resultant pressure should be nearly at right angles to the surface of the joint.

3. The maximum intensity of compression on each joint, including the base, must not exceed the safe compression strength of the material.

\section{§ 2. Masonry Arch. Line of Pressure.}

86. Definitions. In Fig. $70, a$ and $b$ are respectively the span and rise of the arch, $h$ is the thickness of the arch-ring, also the depth of the joints. The inner and outer surfaces of the arch-

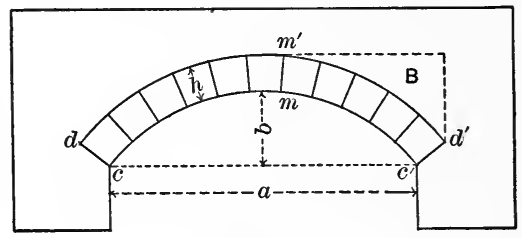

FIG. 70.

ring are the intrados and extrados respectively, these terms being also applied to the lines $c m c^{\prime}$ and $d m^{\prime} d^{\prime}$. The highest part of the arch is the crown. The surfaces $c d$ and $c^{\prime} d^{\prime}$ are the skew-backs. The portions of the arch-ring between the crown and skewbacks are the haunches.

The space $B$, outside the extrados and within the dotted lines, is the spandrel. The masonry, usually with horizontal joints, lying in space $B$ is the backing. The timber frame which supports the arch during erection is the centring.

Arches are designated according to the form of the intrados as semicircular, segmental, elliptical, pointed, etc.

87. Line of Pressure a Funicular Polygon. Let $A B, B C$, $C D$, etc. (Fig. $7 \mathrm{I}$ ), be the loads supported by the arch-stones. If 
the pressure at any joint, as $a$, is given completely, the pressures at the other joints can be found by the triangle of forces (Art. 80). Thus, representing the pressure on the joint $a$ by $P A$, the resultant of $P A$ and $A B$, i.e., $P B$, will be the pressure on the joint $b$, its line of action passing through the intersection of $p a$ and $a b$ as shown. Similarly, $P C$ is the pressure on the joint $c, P D$ on the joint $d$, etc. Thus it is seen that the lines of

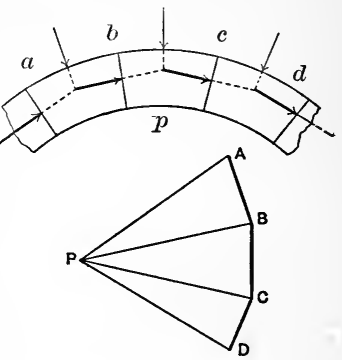

FIG. 7I. action of the resultant pressures on the successive joints of an arch are the strings of a funicular polygon, the corresponding rays representing the magnitudes of these pressures. This funicular polygon will be referred to as the line of pressure of the arch, although the line of pressure or line of resistance, as commonly defined, is the broken line joining the centres of pressure of the successive joints.

When the arch and its loading are symmetrical it is evident that the line of pressure will also be symmetrical with reference to a vertical through the crown, and hence the pressure at the crown will be horizontal. In this case only one-half the archring need be considered.

88. A Test of Stability. In order to satisfy condition (I) of Art. 85 , in case of rectangular joints, it is necessary for the resultant pressure to act within the middle third of the depth of the joints (Art. 84); or, as commonly stated, the line of pressure must lie within the middle third of the depth of the arch-ring. If, then, it is found impossible to draw any funicular polygon for the given system of loads which will satisfy this condition, 
the proposed arch is unsatisfactory and must be altered in one or more of the following particulars: r. Thickness of arch-ring; 2. Form of arch-ring; 3. Distribution of loading.

A method of determining the possibility of drawing a funicular polygon which will satisfy the above condition is illustrated in case of the segmental arch of Fig. 72 .
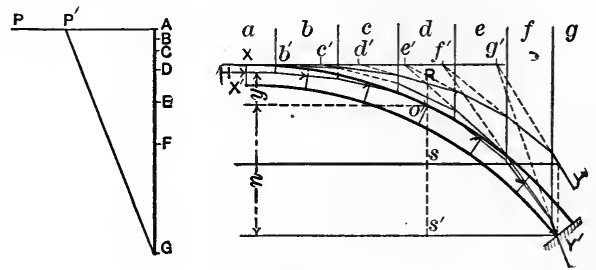

Fig. 72.

The arch is loaded symmetrically, the loads being assumed vertical. The two curves drawn include between them the middle third of the thickness of the arch-ring. The half-arch is divided into equal segments, the loads supported by these being $A B, B C$, etc. The letters $a, b$, etc., will be used to represent the strings of the funicular polygons and also the joints of the arch, $a$ being the joint at the crown and $g$ the joint at the springing. These so-called joints need not be actual joints but only convenient divisions of the arch-ring.

The constructions of either Arts. 23, 24, or 25 may be employed. Using first the construction of Art. 25, we proceed as follows: Assuming any pole $P$ on a horizontal line through $A$, any funicular polygon $X Y$ is drawn, the strings being extended to intersect the string $a$ at the points $b^{\prime}, c^{\prime}$, etc., as shown. These points of intersection locate the resultant loads lying between the corresponding joints (see Art. 25).

We now proceed to seek a polygon whose various strings pierce 
the corresponding joints w thin the limiting curves. Judging from the general form of the polygon $X Y$, it appears probable, for example, that one drawn through the points $X$ and $Y^{\prime}$ will satisfy the condition. The strings $d, e, f$, and $g$ of this polygon are drawn as follows: Draw the string $g$ through $Y^{\prime}$, its direction being $Y^{\prime} g^{\prime}$; then draw $f, e$, etc., in turn so as to pass through $f^{\prime}, e^{\prime}$, etc., as shown. It is observed that this polygon falls outside the required limits, its point of greatest departure being at the joint $d$. It thus becomes apparent that a polygon drawn through $Y^{\prime}$ and touching the outer limiting curve at the joint $d$ is most likely to satisfy the requirement. This polygon might have been constructed by the same method as the preceding one, otherwise by Art. 24 as follows: Treating the polygon $X Y$ and the one to be drawn as one-half the funicular polygons for the whole arch, the closing strings will be horizontal as shown. Since the required polygon is to pass through $O^{\prime}$, the intercepts made by the vertical through $O^{\prime}$ are $R S$ and $O^{\prime} S^{\prime}$ respectively. The services of the new polygon and its pole $P^{\prime}$ are then located as described in Art. 24. This final polygon is seen to satisfy the requirement.

It should be noted that if this final polygon were the true line of pressure, the points, indicated by arrows, where the strings cut the corresponding joints would be the centres of pressure at such joints, the corresponding rays representing the magnitudes of these pressures.

For the case of non-symmetrical loading see Art. 93.

The student should be able to check any graphical work algebraically, as in case of frames. Thus let it be required to determine algebraically the magnitude and location of the crown pressure $H$ (Fig. 72), such that the funicular polygon will pass through $O^{\prime}$ and $Y^{\prime}$.

It is known that any string represents the line of action of 
the resultant external force acting between the corresponding joint and the crown. Thus the string $d$ through $O^{\prime}$ is the line of action of the resultant of $H$ and the loading above the joint $d$. The resultant moment of these forces about $O^{\prime}$ must therefore be zero, i.e.,

$$
H \cdot y=M, \text { • • • • • • • }
$$

in which $M$ is the moment, about $O^{\prime}$, of the loads above the joint d. Similarly,

$$
H(y+n)=M^{\prime} \text {, • • • . • . . }
$$

in which $M^{\prime}$ is the resultant moment, about $Y^{\prime}$, of the loads above the springing.

By solving (I) and (2), the values of $H$ and $y$ may be obtained.

89. Relation between Line of Pressure and Form of Archring. It is evidently desirable, to insure stability, that the true line of pressure coincide, as nearly as possible, with the centre line of the arch-ring.

If various funicular polygons be constructed for the same system of loads, changing the position of the pole but keeping it on the same horizontal line, all such polygons will have the same general form; hence, since one such polygon constitutes the true line of pressure, the most suitable form of arch for a given system of loads may be determined by observing the general form of funicular polygons for such loads. Thus, in Fig. 72, the form of the polygon $X Y$ or $X^{\prime} Y^{\prime}$ would suggest the elliptical or the multiple-centred circular arch to be the best forms for that case. The most suitable form for a load uniformly distributed horizontally would be parabolic, since the funicular polygon for such loading is a parabola (Art. 13). And, in general, an arch comparatively flat at the crown is suitable when the load increases in intensity from the crown towards the abutments, while a pointed arch is better adapted to the reverse condition. 
Conversely, when the form of arch is given, its most suitable distribution of loading can be determined in a general way by treating its centre line as a funicular polygon and determining the corresponding distribution of loads. The method is illustrated in Fig. 73.
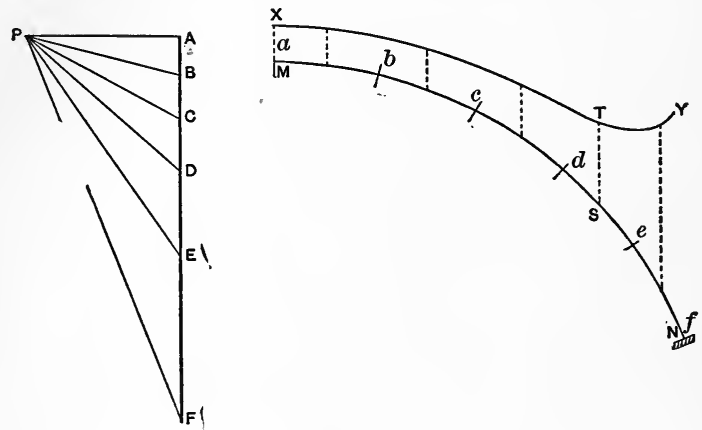

Fig. 73.

Let $M N$ be the centre line of the arch-ring, the short lines $a, b$, etc., being the joints and the dotted lines indicating the lines of action of the loads. The strings of a funicular polygon to coincide with $M N$ will be tangent to the curve at the points $a$, $b$, etc. Therefore, to determine the distribution of loading corresponding to this funicular polygon, draw from any pole $P$ lines $P A, P B$, etc., parallel to tangents at $a, b$, etc. The lengths $A B, B C$, etc., thus determined indicate the relative magnitudes of the loads on the divisions $a b, b c$, etc., of the arch-ring. This distribution of loads may be represented by laying off ordinates such as $S T=D E$, etc. The area between the curve $X Y$ thus constructed and $M N$ represents the distribution of loading. It should be observed that the diagram only represents the desired clistribution of loads in a general way, since it is not known that $M N$ is the true line of pressure. 
90. Maximum and Minimum Crown Pressure. It is evident that as the pole distance (i.e., pressure at the crown) increases, the rays and strings of the funicular polygons become more nearly horizontal and consequently the polygons become more nearly flat. Hence, of all polygons which can be drawn within the middle third (or other designated limits) of the arch-ring, that one will correspond to a maximum crown pressure which touches the inner limiting curve at a point nearer the crown than that at which it touches the outer curve, and conversely.

Thus, in Fig. 72 . the final polygon $X^{\prime} Y^{\prime}$ corresponds to minimum crown pressure $\left(P^{\prime} A\right)$, while the one corresponding to maximum crown pressure would touch the inner limiting curve at the crown and the outer limiting curve at some point nearer the springing.

It should be noted in this connection that, other things equal, as the rise of an arch increases the crown pressure diminishes.

It should also be noted that when three points on the line of pressure (corresponding to the thrce hinges of a three-hinged arch) are given, the magnitude and direction of the resultant pressure at such points can be determined algebraically in a manner similar to that explained in Art. $5^{2}$.

91. Location of the True Line of Pressure. It remains to consider the question as to which of the infinite number of funicular polygons corresponding to a given system of arch loads constitutes the true line of pressure.

The prominent methods ${ }^{1}$ which have been suggested or are in use for locating the true line of pressure may be divided into two classes, namely:

I. Methods based on the "Theory of the Elastic Arch." 2

\footnotetext{
'See Baker's "Masonry Construction" for discussion of the various methods.

'See Weyrauch's "Theorie der Elastigen Bogenträger"; also, Lanza's "Applied Mechanics."
} 
II. Methods not deduced mathematically, but rather conventional, in character, the only substantial evidence of their reliability being apparently derived from observation and experience in their use. These two classes of solutions will be discussed briefly.

Class I. It has been frequently suggested that a masonry arch be treated in the same manner as a continuous iron arch fixed at the ends, the solutions for the latter case being based on the "Theory of the Elastic Arch." These solutions ${ }^{1}$ may be designated as follows:

A. The exact solution, in which the general formulas derived from the "Theory of the Elastic Arch" are employed. The line of pressure thus located may be taken to conform within very slight limits of error to the theory. This solution is very laborious.

B. The approximate solution, in which all but one of the terms of the general formulas are omitted, leaving them in the following form:

$$
\Delta \phi=\int \frac{M_{x}}{E I} d s ; \Delta x=-\int \frac{M_{x}}{E I} y d s ; \Delta y=+\int \frac{M_{x}}{E I} x d s .
$$

This method is the one commonly presented in text-books. It is adapted to graphical methods of solution.

C. A proposition by Dr. Winkler forms the basis of a solution which may be properly included under Class I, since it is derived from the formulas of solution B. This proposition is as follows: "For an arch of constant section, that line of resistance (pressure) is approximately the true one which lies nearest the axis of the arch-ring as determined by the method of least squares."

No approximate method should be employed without first 
ascertaining the amount of error involved. For this purpose a comparison of the results to be obtained by the use of methods A, $\mathrm{B}$, and $\mathrm{C}$ was made in the cases shown in Plate III. Two extreme cases were taken, namely: I. A semicircular arch; 2. A segmental arch whose ratio of rise to span is I : Io. In each case three systems of loads were employed, namely: I. A concentrated load at the crown; 2. A load uniformly distributed over the entire span; 3. A load uniformly distributed over the half-span.

The arches were of uniform thickness (shown at the crown), ratio of span to thickness of arch-ring being about ir.6. The lines of pressure (C) were located by the calculus, not by trial, so as to conform exactly with Winkler's proposition.

The results will not be discussed in detail, but the following conclusions seem to be warranted.

I. Method $\mathrm{C}$ gives in each case (except Case 4) a line of pressure deviating to such an extent from that of Method A as to prove the unreliability of the former.

2. Method $\mathrm{B}$ gives a line of pressure agreeing closely with (A) in case of the semicircular arch, but deviating considerably from the latter in the case of the segmental arch. It is evident that Method B must be used with discrimination if the results are to be trustworthy. In general, the greater the ratio of span to thickness of arch-ring and the greater the ratio of rise to span, the less will be the difference between the results obtained from Methods A and B.

Class II. Of these, the method of "Least Crown Pressure" seems to be most commonly employed. ${ }^{1}$

The theory of least crown pressure is essentially that the true line of pressure is that one which, lying within the middle third (or other designated limits) of the arch-ring, corresponds to a

${ }^{1}$ See Baker's "Masonry Construction." 
minimum crown pressure (see Art. 90). It appears to be based upon the observation that most arches settle at the crown when the centring is removed, and upon the consideration that the crown pressure is a passive force developed by the tendency of the two half-rings to tip towards each other, and is the least force that is necessary to prevent such overturning.

If the arch settles at the crown (as a result of rotation, not sliding, of the arch-stones), the tendency would appear to be to open the joints as shown in Fig. 74, the centre of pressure moving

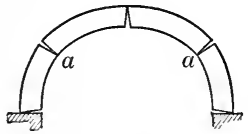

FIg. 74.

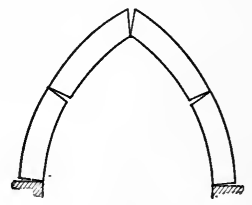

FIG. 75 .

upward at the crown and inward at the haunches. The resulting position of the line of pressure would thus agree in a general way with the case of minimum crown pressure (Art. 90). (It will be observed that all the lines of pressure A, Plate III, also lie above the centre of the arch-ring at the crown and within it at points nearer the abutments.)

The joints $a, a$, where the tendency to open at the extrados is greatest, are called the joints of rupture. They correspond to the points where the line of pressure corresponding to minimum crown pressure touches the inner limiting curve.

In the case of a pointed arch, or an arch very lightly loaded at the crown and heavily loaded at the haunches, the tendency may be for the crown to rise and the haunches to move inward (Fig. 75).

The various other methods of Class II differ from the preceding only in details. Thus the true line of pressure may be 
assumed to be that one which intersects the crown joint at the middle of its depth; at one-third the depth from the extrados, etc.; satisfying, also, other conditions of a similar character. Apparently all such conditions are substantially expressions of judgment only.

Comparison of the Methods of Classes I and II. The fact that the masonry arch is built up of blocks instead of the material being continuous and homogeneous, as in case of the elastic arch, renders it questionable as to whether the same methods of solution are applicable to both. It must also be recognized that the conditions which determine the location of the line of pressure of a masonry arch are commonly numerous and uncertain. Among such conditions are evidently the following:

x. The loading. This is generally uncertain in amount, distribution, and direction. In the case of arches supporting masonry walls the uncertainty is particularly great, but it evidently exists to some extent in all cases.

2. The behavior of the arch as to the kind and amount of distortion occurring when the centring is removed and subsequently. Evidently its behavior will depend upon the quality of the material and workmanship, the rigidity of the supports, etc., as well as upon the loading.

It is evident that reliable formulas cannot be derived from uncertain data, however correct the reasoning may be. Under such circumstances the final test of the reliability of any method of solution must be agreement with the results of experiment and experience. In this respect the methods of Classes I and II stand on the same footing, as each requires experimental verification.

For the present, in lieu of adequate experimental results we must rely largely upon the experience of the past in the construction of masonry arches; making use of that line of pressure 
which appears to be most reliable and seeing to it that, with such line of pressure, the conditions of Art. 85 are fulfilled.

The author has no recommendation to make as regards the best method of locating the line of pressure of a masonry arch. In the following examples, however, the condition that "the true line of pressure is that one which, lying within the middle third of the arch-ring, corresponds to the minimum crown pressure," will be employed; the portion of the solution subsequent to the location of the line of pressure being the same, however this line may be located.

In order to fix the position of the line of pressure, three hinges have been employed to some extent in Germany and elsewhere. The line of pressure is thus determined by the condition that it must pass through the hinges, the same as in case of the three-hinged iron arch.

92. Example. Figure I, Plate III, is one-half of a symmetrical full-centred arch in a masonry wall whose height is limited by a horizontal line, as shown. It is required to draw the line of pressure according to the theory of least crown pressure, and to determine if the arch satisfies the conditions of stability.

The half-ring is divided by radial lines, which need not coincide with the actual joints of the arch. It will be assumed that each of these divisions supports the weight of the portion of wall directly above it, as indicated by the vertical lines. If the specific gravity of the material above the arch-ring is the same as that of the arch-ring, the load supported by any single division, as $m n$, is proportional to the area of the polygon $m n n^{\prime} m^{\prime}$. If the specific gravities are unequal, the vertical ordinates may be altered in length so that the areas above the divisions will represent weights to the same scale as the areas of the divisions themselves. Otherwise the weights of the divisions and material 
above them may be dealt with separately. In this example, the wall is of uniform thickness and the weight of the masonry is, throughout, r6o lbs. per cubic foot.

Considering one foot thickness of wall, the loads (see table)

TABLE OF LOADS.

(Fig. I, Plate III.)

\begin{tabular}{|c|c|c|}
\hline Division. & $\begin{array}{c}\text { Weight } \\
\text { of } \\
\text { Division. }\end{array}$ & $\begin{array}{c}\text { Weight } \\
\text { above } \\
\text { Division. }\end{array}$ \\
\hline$A B$ & Lbs. & Lbs. \\
$B C$ & 540 & $\mathrm{I} 360$ \\
$C D$ & 540 & 1440 \\
$D E$ & 540 & 1570 \\
$E F$ & 540 & 1700 \\
$F G$ & 540 & $\mathrm{I} 790$ \\
$G H$ & 540 & $\mathrm{I} 760$ \\
$H I$ & 540 & 1540 \\
$I J$ & - & 1680 \\
$J K$ & - & 7680 \\
\hline
\end{tabular}
are calculated by multiplying the corresponding areas by $\mathrm{I} 60 \mathrm{lbs}$. $H I$ is taken to include two divisions to avoid confusing the drawing; $I J$ is the weight of the masonry to the right of the line $i ; J K$ is the weight of the masonry below $R S$.

The resultant loads act at the centres of gravity of the areas. The centre of gravity of the division $m n$ is $O^{\prime}$, and the centre of gravity of the trapezoid above this division is $O^{\prime \prime}$ (see Art. 79). The centre of gravity $O$ of the entire area $m n n^{\prime} m^{\prime}$ is then found by dividing the line $O^{\prime} O^{\prime \prime}$ into parts inversely proportional to these areas. The centres of gravity are indicated by circles.

The line of pressure is now constructed as follows: The loads $A B, B C$, etc., are plotted to scale, and, selecting any pole $P$ on a horizontal line through $A$, a preliminary polygon $x y$ is drawn. the point $x$ being one-third the depth of the joint below the extrados. In drawing this polygon, the intersections $c^{\prime}, d^{\prime}$, etc., of its various strings with the string $a$ are marked (see Art. 88).

The line of pressure desired is such that the resultant pressure at the joint of rupture will act at one-third the depth of the joint from the intrados. The joint of rupture may be determined by trial as follows: Judging that it will be near the joint $g$, we trisect that joint at $I$ and draw the string $g$ through $I$ and $g^{\prime}$. The adjacent strings (not shown in the drawing) are then drawn, and 
it is found that the string $f$ falls farthest outside the middle-third limit. This joint is then trisected at 2 and the string $2 f^{\prime}$ of a new polygon is drawn. This polygon is completed by drawing the remaining strings in succession through $e^{\prime}, d^{\prime}$, etc., and is found to satisfy the conditions except near the springing where it falls outside the middle-third limit. This portion of the arch, however, can be treated as part of the abutment. The centres of pressure at the different joints are indicated by arrows.

Aside from the condition that the pressure must act within the middle third of the arch-ring, the resistance to sliding and crushing must be investigated.

As regards sliding, it is seen that the direction of the resultant pressure at each joint is very nearly normal to the joint, with the exception of the springing plane, where the pressure $p^{\prime} i$ makes with the normal an angle greater than $\tan ^{-1} \cdot 4$. When, however, the weight $I J$ is combined with $P^{\prime} I$, the resultant pressure, $P^{\prime \prime} J$ (Art. 96), satisfies the requirement for safety against sliding.

For crushing, the maximum compression stress is to be calculated at each dangerous joint. For example, the resultant stress on the joint $f$, found by scaling off the ray $P^{\prime} F$, is 13 Ioo lbs. The area of the surface of the joint is $\mathrm{I} \times \mathrm{I}_{\frac{3}{4}}=\mathrm{I}^{\frac{3}{4}} \mathrm{sq} . \mathrm{ft} .=$ $25^{2}$ sq. in. Average pressure per sq. in. $=\frac{13 \frac{13}{2} 5^{2} 0}{2}=5^{2} \mathrm{lbs}$. Hence the maximum stress $=2 \times 5^{2}=104$ lbs. per sq. in. (see Art. 84). This stress must not exceed the working compression strength of the masonry. (For strength of masonry see Lanza's "Applied Mechanics" and other references.)

93. Unsymmetrical Cases. When the arch or loading is unsymmetrical, the line of pressure is also unsymmetrical, and must therefore be drawn for the whole arch. The construction of the line of pressure involves the problem of drawing a funicular polygon through three points (see Arts. 23 and 24). 
Example. (Fig. 76.) Given a segmental arch of $16 \mathrm{ft}$. span and $3 \mathrm{ft}$. rise. Thickness of arch-ring $=I_{\frac{1}{4}} \mathrm{ft}$. The left and right halves are loaded with $3200 \mathrm{lbs}$. and $6400 \mathrm{lbs}$. respectively, per foot width of arch, these loads being uniformly distributed over the arch-ring. It is required to construct, if possible, the line of pressure in accordance with the method of "least crown pressure."

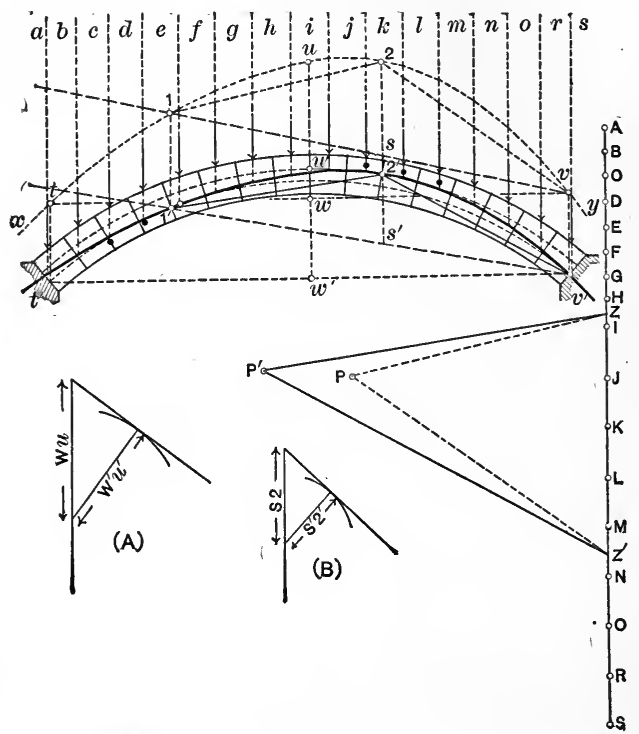

Frg. 76.

The arch-ring is divided into sixteen equal divisions, and the load supported by each is assumed to act at the middle of its outer surface. The loads are plotted to scale, and the funicular polygon $x y$ is constructed, using $P$ for pole. Selecting the points $t^{\prime}, v^{\prime}$, and $u^{\prime}$ at one-third and two-thirds the depth of the joints from 
the intrados, the funicular polygon which will pass through these points is located as explained in Art. 24 (see Fig. 76 A). This polygon is not drawn, but the points on it, falling outside the middle-third limits, which serve to locate the final line of pressure, are marked by dots.

It is seen that to the right of the crown this polygon rises above the middle-third limit, while near the left abutment it falls below. From the position of these points it appears probable that a polygon drawn through the three points $I^{\prime}, 2^{\prime}$, and $v^{\prime}$ will fall within the specified limits. The vertices of this polygon (drawn in full lines) were located in the same manner as the preceding one (see Fig. $76 \mathrm{~B}$ ), and it is seen that the centres of pressure at all the joints fall within the required limits.

The pole $P^{\prime}$ of this final polygon was located by the method of Art. 23, i.e., $P Z$ and $P Z^{\prime}$ were drawn parallel to 1,2 and 2, $v$ respectively; then $Z P^{\prime}$ and $Z^{\prime} P^{\prime}$ were drawn through $Z$ and $Z^{\prime}$ parallel to $I^{\prime}, 2^{\prime}$ and $2^{\prime}, v^{\prime}$ respectively, thus locating $P^{\prime}$. This pole might also have been located by Art. 24. The resultant compression at the various arch sections is represented by the rays of this final polygon.

The stability of the abutments is essential to that of the arch (see $\$ 3$ ).

\section{§3. Abutments, Piers, etc.}

94. Conditions of Stability. The general conditions of stability of $\S$ I are applicable to any piles of masonry subjected to the action of external forces, such as the thrust of an arch or truss, pressure of earth, water, wind, etc.

95. Example 1. Fig. 77 is an abutment subjected to a horizontal pressure $A B$ and a vertical pressure $B C$, their re- 
sultant. $A C$, acting at the point c. $C D, D E, E F$, and $F G$ are

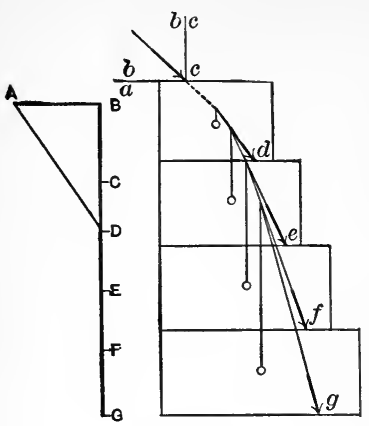

FIG. 77.

the weights of the four divisons of the masonry, their centres of gravity being marked by circles. The pressure on the joint $d$ is the resultant of $A C$ and the weight $C D$ of the first block. This resultant is $A D$, its line of action passing through the intersection of $a c$ and a vertical through the centre of gravity of the block. The point of application of this resultant pressure,

at $d$, is indicated by an arrow. The resultant pressures at the remaining joints are found in a similar manner.

The conditions of safety as regards sliding, overturning, and crushing have been previously discussed. The maximum pressure on the soil must also be kept within safe limits (see Baker's “Masonry Construction," Chap. X).

A broken line connecting the points of application $c, d, e, f$, and $g$ of the resultant pressures on the successive joints of an abutment is commonly called the line of resistance or line of pressure, as in case of an arch (see Art. 87).

96. Example 2. The abutments of a masonry arch can be considered in connection with the arch. In Fig. I, Plate III, the weights $I J$ and $J K$, lying above and below the joint $R S$, are plotted to half scale $(4000 \mathrm{lbs} .=\mathrm{I}$ in.), the pole for these two loads being located by bisecting the ray $P^{\prime} I$ at $P^{\prime \prime}$. The resultant pressure on $R S$ is $P^{\prime \prime} J$, its line of action $p^{\prime \prime} j$ passing through the intersection of $p^{\prime} i$ and $i j$. The resultant pressure on the base is $P^{\prime \prime} K$, its line of action being $p^{\prime \prime} k$. To find the maximum intensity of 
the pressure on the base we have $F^{\prime \prime} K=9$.I (inches) $\times 4000=$ $36400 \mathrm{lbs}$. By measurement, $p^{\prime \prime} k$ acts I.I ft. from the centre of the base. The bearing area is $7 \frac{3}{4} \times I=7 \frac{3}{4}$ sq. ft. Substituting these values in the formula $f=\frac{P}{A}+\frac{M}{I} y$, we have

$$
\begin{aligned}
f=\frac{36400}{7 \cdot 75}+\frac{36400 \cdot 1 \cdot I}{\frac{1}{12} \cdot I \cdot(7 \cdot 75)^{3}} \cdot \frac{7 \cdot 75}{2} & =8696 \text { lbs. per sq. ft. } \\
& =60 \text { lbs. per sq. inch. }
\end{aligned}
$$

97. Example 3. Let Fig. 78 represent a pier supporting the thrust of an arch on each side. These thrusts are $A E$ and $B C$, their point of intersection $O$ lying on the centre line of the pier. Let $C D, D E, E F$, and $F G$ represent the weights of the pier divisions, $C D$ being the weight of the pier masonry above the joint $d$. The resultant pressure on $d$ is $A D$, its line of action $O d$ passing through $O$. The lines of action of the pressures on the remaining joints will also pass through $O$, since the vertical

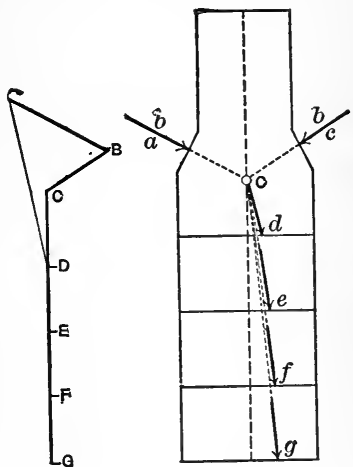

Fig. 78 . through this point contains the centres of gravity of all the blocks. These lines are $O d, O e, O f$, and $O g$, drawn parallel respectively to $A D, A E, A F$, and $A G$. If the thrusts of the two arches are equal and equally inclined, the resultant pressure on the pier will evidently be vertical.

98. Example 4. Let Fig. 79 represent a chimney subjected to wind pressure. The weights of the portions $a b, b c$, etc., are 
$A B, B C$, etc., and the wind pressures on these portions are $A B^{\prime}$, $B^{\prime} C^{\prime}$, etc. The lines of action of these wind pressures are the horizontal dotted lines $a b, b c$, etc. With any pole $P$ draw the funicular polygon $m n$ for the wind loads. (For this purpose these loads should be plotted to a larger scale.)
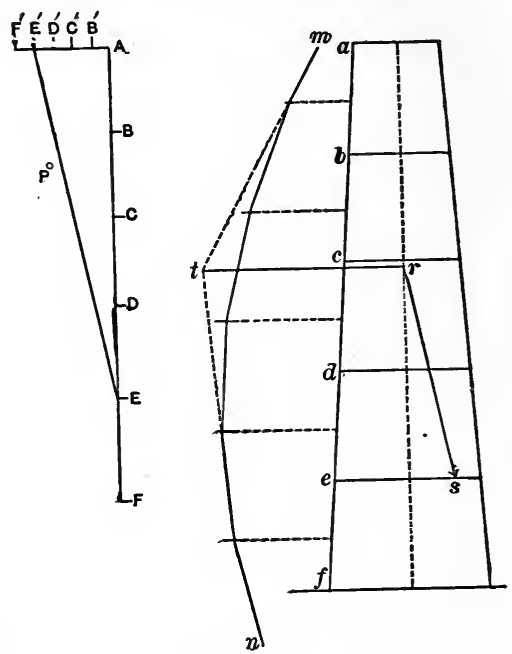

FIG. 79.

To find the resultant pressure at any section, as $e$, the line of action $t r$ of the resultant wind pressure above that section is located by the intersection of the strings $a$ and $e$.

The pressure at the section $e$ is the resultant of this wind pressure and the weight of masonry above $e$. The line of action $r s$ of this pressure will act through $r$, the point of intersection of the resultant wind pressure and weight of masonry above $e$, and its direction will be parallel to $E^{\prime} E$, its magnitude being repre- 
sented by the length of $E^{\prime} E$. The resultant pressure at any other section can be determined in a similar manner.

The preceding examples will serve to indicate the method of determining the stability of such structures when the loads are known.

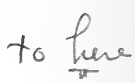





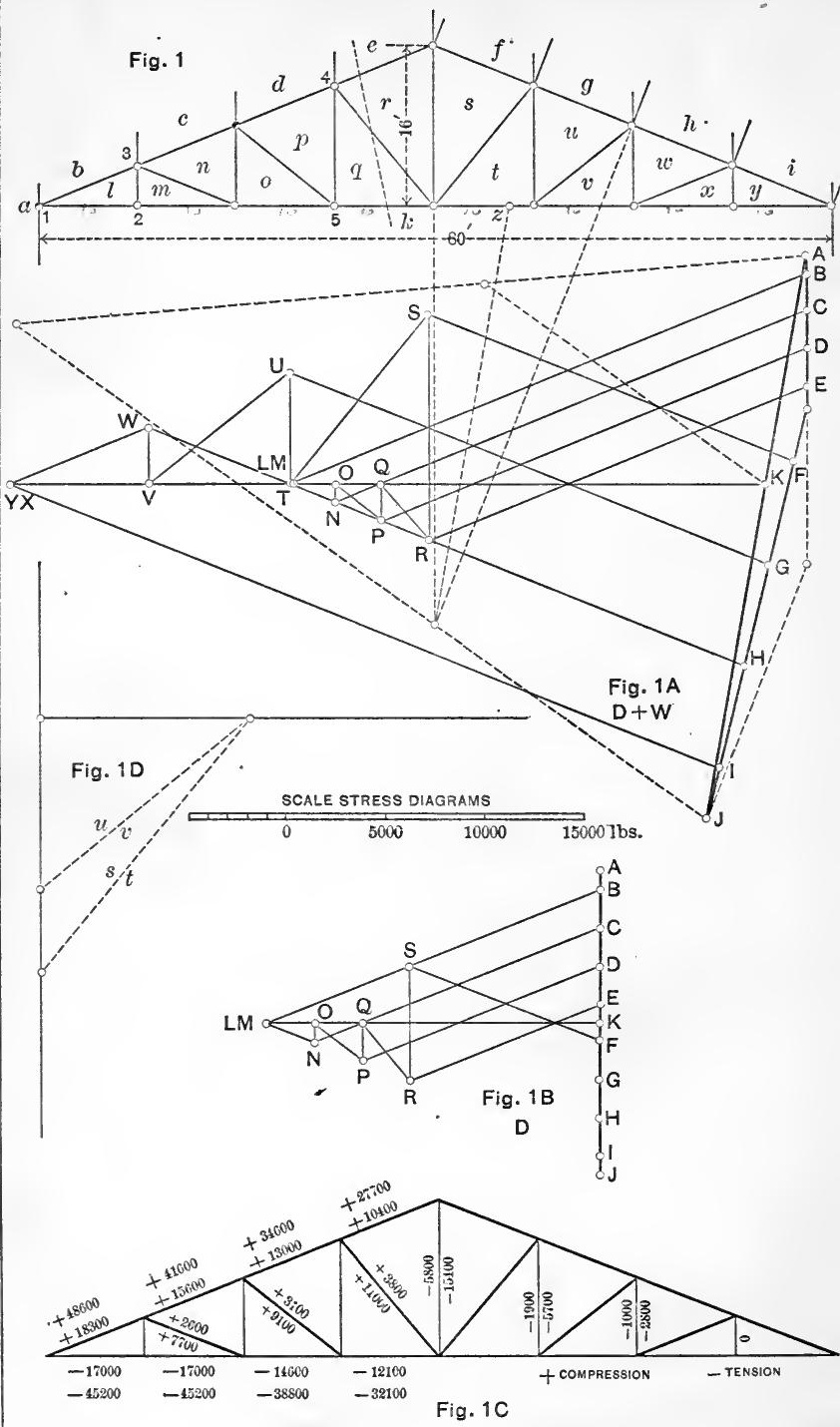


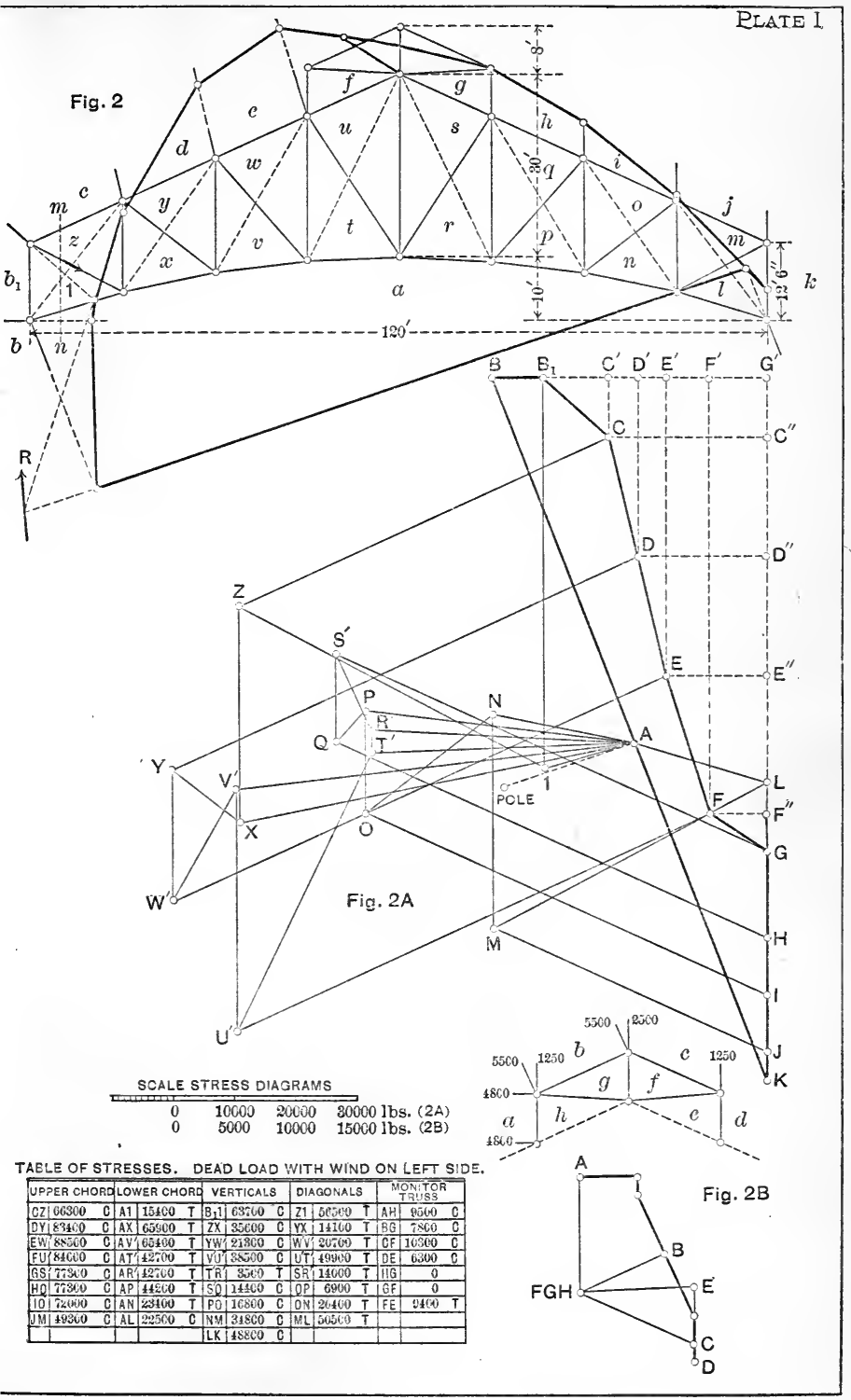






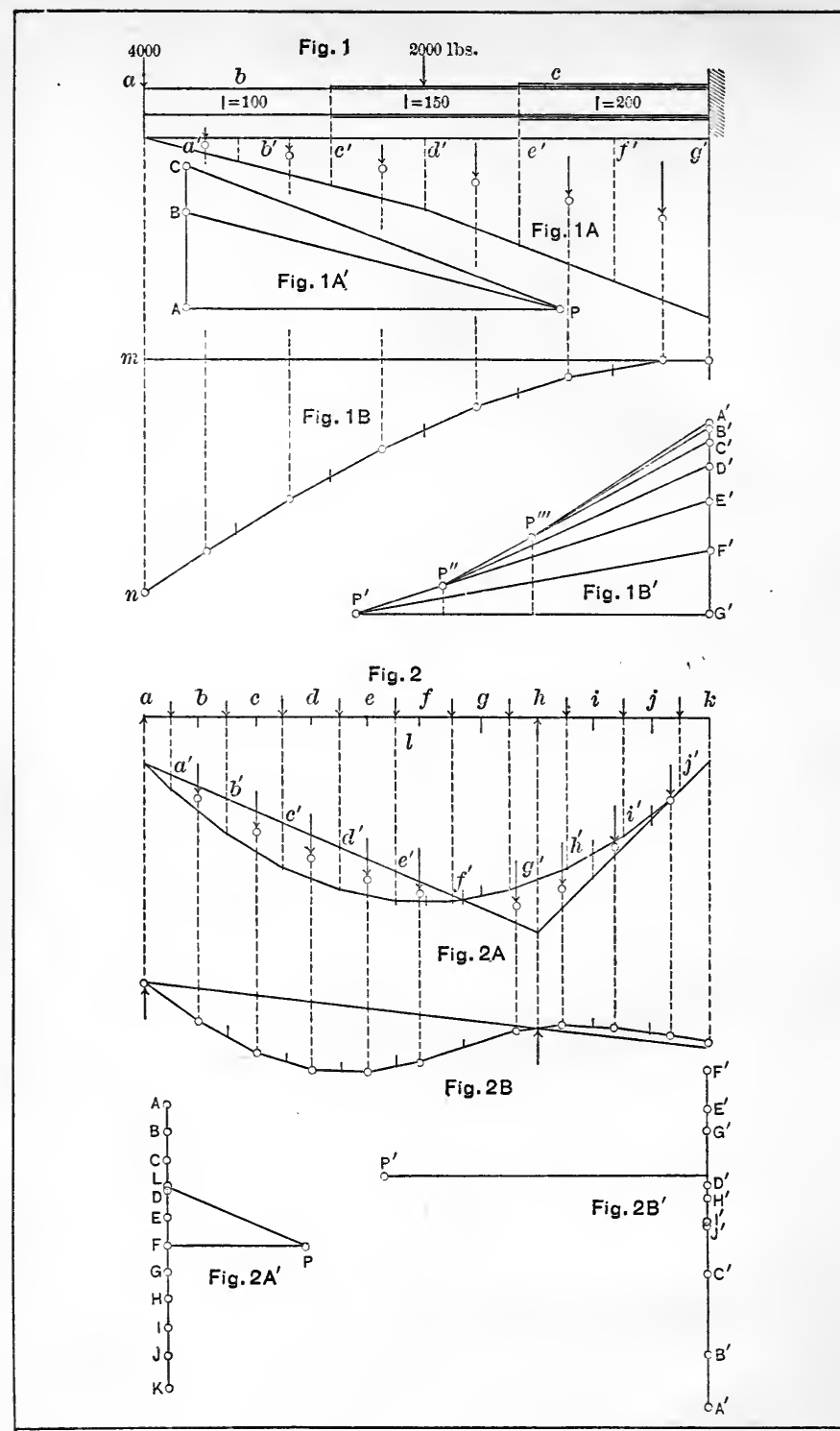




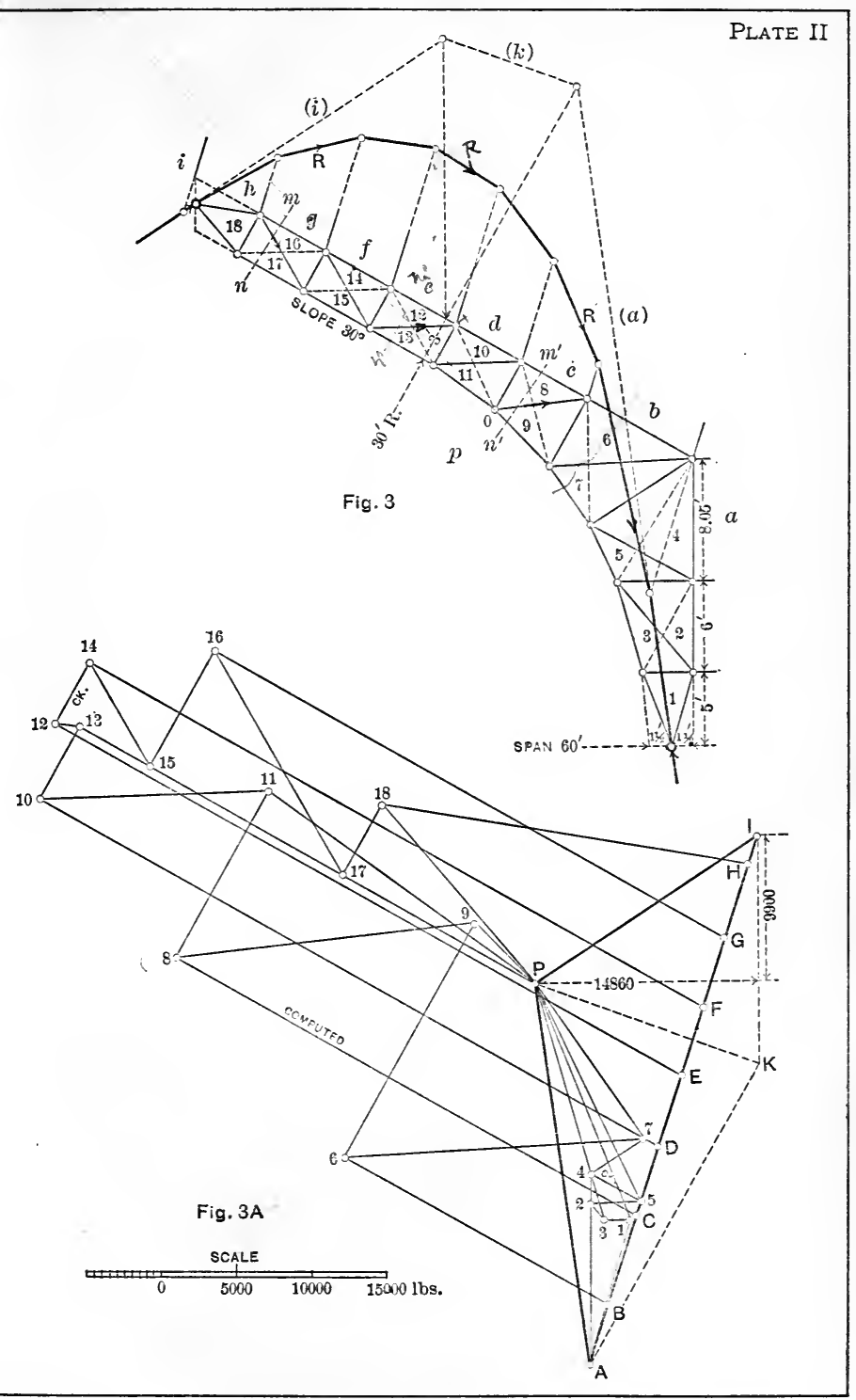






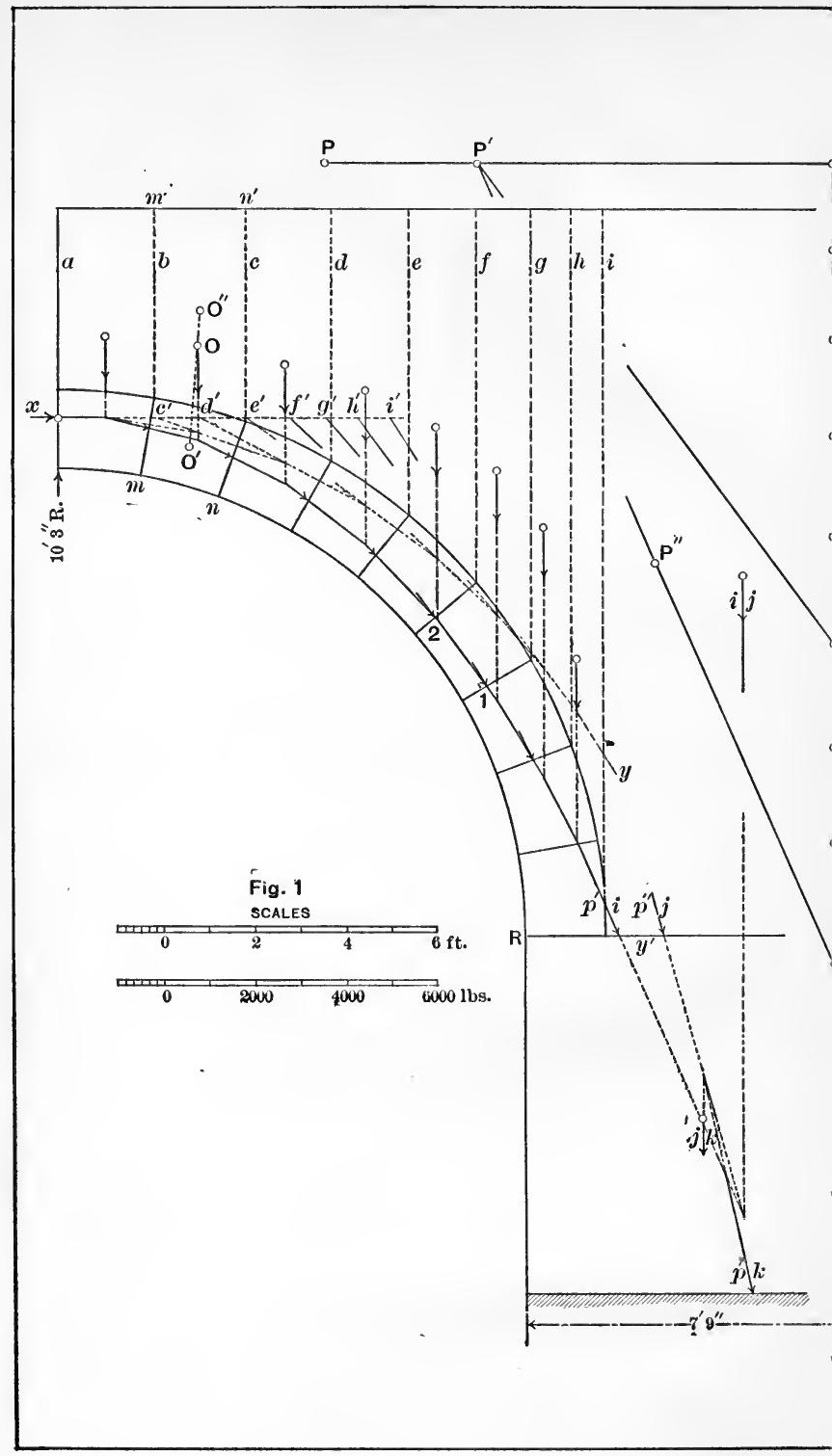




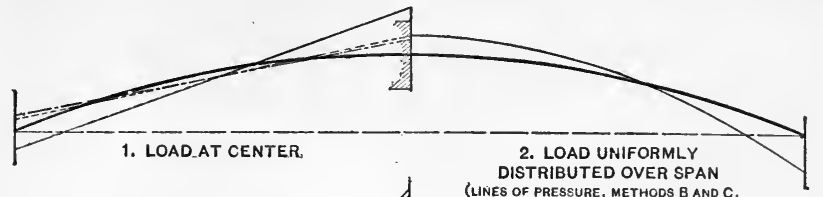
DISTRIBUTED OVER SPAN

(LINES OF PRESSURE, METHODS B AND C, COINCIDE CLOSELY WITH AXIS OF ARCH RING)

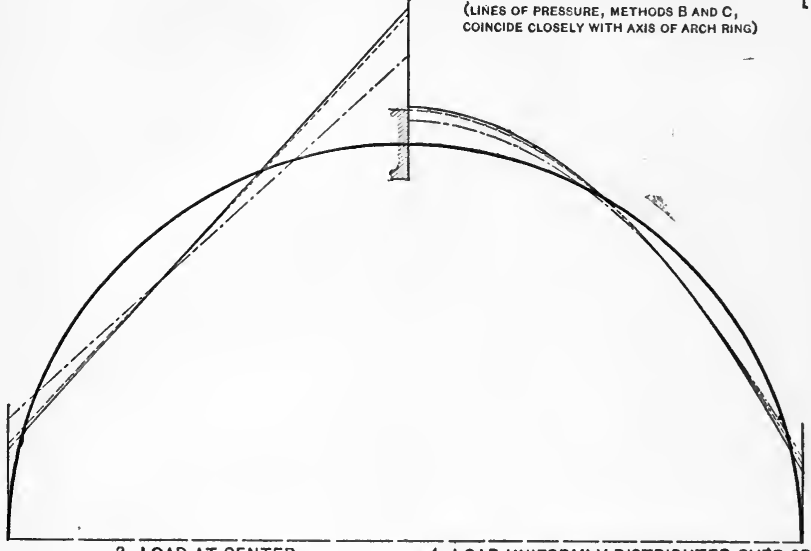

3. LOAD AT CENTER,
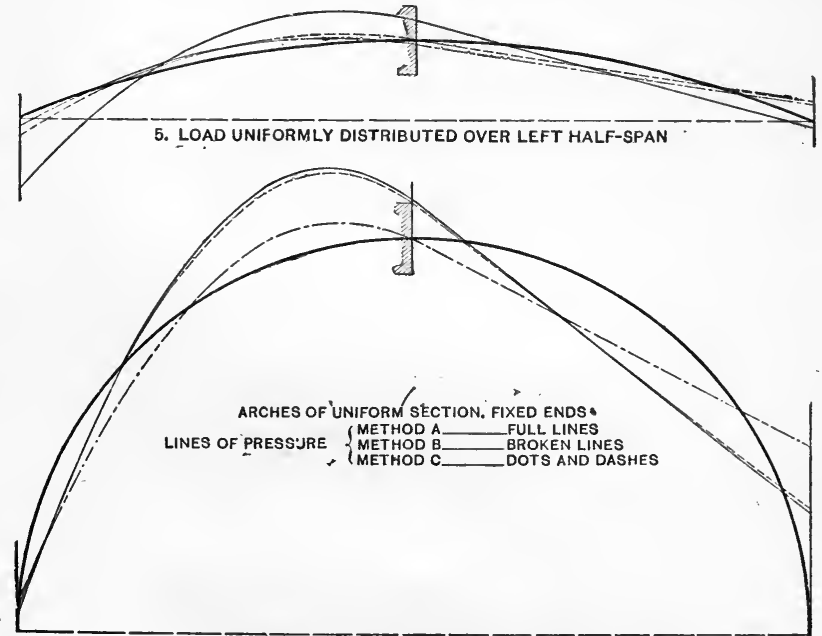

6. LOAD UNIFORM $\overline{L Y ~ D I S T R I B U T E D ~ O V E R ~ L E F T ~ H A L F-S P A N . ~}$ 





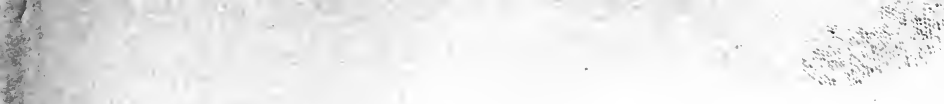

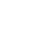


TE

THE LIBRARY

UNIVERSITY OF CALIFORNIA

Santa Barbara

THIS BOOK IS DUE ON THE LAST DATE STAMPED BELOW.

$50 m-3,68(11924258) 9482$ 


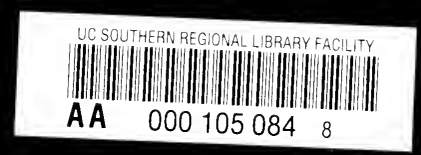


